https://doi.org/10.31713/m1106

\title{
SOLUTIONS OF POLYMERS IN THE OIL AND GAS TECHNOLOGIES
}

\author{
Pogrebnyak V. G.
}

Ivano-Frankivsk National Technical University of Oil and Gas, Professor, Doctor of Technical Sciences, Head of the Department of Technogenic and Environmental Safety, Ukreine

\section{Chudyk I. I.}

Ivano-Frankivsk National Technical University of Oil and Gas, Professor, Doctor of Technical Sciences, Vice-Rector for Research, Ukreine

Perkun I. V.

Ivano-Frankivsk National Technical University of Oil and Gas, $\mathrm{PhD}$ (Engineeuing), Associate Professor of the Department of Technogenic and Environmental Safety, Ukreine

\begin{abstract}
There has been carried out a complex study of polymer solution reaction under hydrodynamic effect. Integrated approach turned out to be a necessary condition for successful solving of the set tasks of scientific and technical character (from fundamental studies to concrete technological process). This allowed to formulate a new structural concept with a "common denominator" such as strong deformation effect on the part of a hydrodynamic field on macromolecules that immediately generates the so called rubber-like high elasticity. The technologies of production oil and gas is based on utilization of rubberlike properties of a polymer solution that arise under definite conditions while going through porous media, nozzles and slotes. There have been obtained eco-technological criteria and practical recommendations as to practical implementation of technologies of production oil and gas.

\section{Introduction}

Last years in connection with the ripening ecological crisis there has grown practical interest to creating new nature protection technologies or ecological technologies called so. One of the most perspective directions to solve nature protection problems is a wide usage of "abnormal" phenomena revealed in the conditions of complex hydrodynamic influence on liquids with additives of soluble flexiblechain high-molecular polymers. Here it is necessary to recollect the following "abnormal" phenomena.
\end{abstract}


It is more convenient to start with the decrease of turbulent friction due to small polymeric additives. Let's take advantage of analogy with speed-boats and in general, so to say, with cigar-shaped floating bodies - dolphins or high-speed fish. On border of any body moving quickly in water when Reynolds' critical number is being in excess there appears wall-adjacent turbulence bringing resistance to movement in addition to a common viscous friction. The turbulent contribution to resistance makes tens of percent from the overall resistance. Dolphins, other cetacean as well as high-speed fish in the course of biological evolution have developed the mechanisms that relieve if not all, but a considerable part of turbulent resistance. In case of dolphins it is a kind of surface "counter-waves", reducing wall-adjacent turbulence with mucous excreta. It is characteristic that Toms' effect is being revealed at insignificant additives (million mass fractions) of very high-molecular polymers (a $10^{7}$ order of molecular weight).

Despite the application of modern knowledge and methods, yet it was not possible to describe precisely the mechanism used by fish to produce motive power, and in many respects it is still unknown. At the same time it is known that slime of fish can function as effective high-molecular substance reducing turbulent friction. Polymeric substance in the form of slime can be one of means used by fish to reduce their turbulent friction (valid in case of other high-speed floating animals).

On the basis of the proved strong deformation influence of walladjacent turbulence on macromolecules, and also using data of modeling research of features of turbulence in a frontier layer, one of the authors of the given scientific work has offered the following mechanism of Toms' effect. The mechanism of Toms' effect consists in occurrence of a self-oscillatory mode of reversible processes of macromolecule uncoiling under effect of quasi-regular longitudinal velosity gradients in a turbulent frontier layer and influence of uncoiling macromolecules both on molecular (when $C<C_{\text {opt }}$ ) and supermolecular (when $C>C_{\text {opt }}$ ) levels on the structure of wall-adjacent turbulence. All this leads to increase in the period of liquid emissions in an external part of an interface and, as consequence, to a thickening of a viscous intermediate layer. As a result generation of primary turbulence decreases and the general level of turbulent dissipation in a 
stream goes down. When molecular weights and concentrations are big enough viscosity growth caused by both "usual" intermolecular interaction and dynamic structurization leads to sharp reduction of Toms' effect $[1,2]$.

It is also necessary to stress that modeling experiments have confirmed validity of applying results obtained while studying macromolecule dynamics in non-turbulent flows with stretching to jet flows of wall-adjacent emissions of a turbulent stream, and it means that turbulent (in macroscopic scale) flow is treated as laminar (in microscale) under interaction of a hydrodynamic field with polymer molecules.

There is a possibility of Toms' effect practical application, namely when developing energy-saving technologies, after the nature of turbulent friction decrease with small polymeric additives has been determined.

Another "abnormal" phenomenon, abnormal only from the point of view of classical hydrodynamics, occurs in polymer solutions flowing in porous media. Given "anomaly" is connected with nonlinearity of polymer solutions filtration current and becomes apparent when dissipation energy, hence resistance to a current, at some threshold values of filtration speed starts to increase, and the process is more drastic the higher polymer concentration and molecular weight. Absence of resistance growth to a current of pure solvent with growth of feed velocity testifies to the fact that the effect specified is not connected with inertial turbulence.

Movement of liquids through porous materials serves as the major object for a number of areas of engineering activity. The knowledge of laws of this movement is necessary in oil industry and in soil mechanics, in hydrology and engineering ecology. Non-trivial physical effects lie at the heart of underground hydrodynamics of polymer solutions. In this connection, it is important to know the nature of these effects. Therefore determination of the physical mechanism of displaying effects of sharp increase of hydrodynamic resistance and "abnormally" high degree of porous layer coverage at its filling with a polymer solution was one of the primary goals successfully solved by the authors of this scientific work.

The approach based on deformation influence of a hydrodynamic field on macromolecules in conditions of a filtration current occupies 
special place among attempts to explain the nature of deviations from Darcy's law. To substantiate this approach it is necessary to prove experimentally the presence of strong deformation effect of a hydrodynamic field on molecular coils. Mistrust to the given idea sharply decreased after rather fundamental measurements of a double refraction factor under super-critical flow modes that allowed to prove the possibility of almost full uncoiling of macromolecules and, as consequence of this, phase transition from coil to unfolded chain in a longitudinal hydrodynamic field*.

In brief the problem essence is reduced to the fact that any state of a polymer on a phase plane or in phase space is defined by independent, though interdependent contributions of separate macromolecules (small systems after Hill) and a combination of these macromolecules (big system). In general this approach is substantiated in works by de Gennes, Prigozhin and his followers, and in Keller's works as well.

Meanwhile the whole problem of polymer solutions flow in porous media, nozzles and slotes is brought to the following main principle. Elongation degree (or convolution degree) of a flexible macromolecule can be characterized with the parametre $\beta$, equal to the ratio of distance between the ends of a macromolecule $h$ to its contour length $\mathrm{L}$. In based systems there exists rather simple correlation between the most probable value $\beta \equiv\langle\beta\rangle$ ( $\beta$ is distributed due to Maxwell's law) and Flory's parametre $f$.

However, as it was shown by S. Ya. Frenkel [3] with colleagues and independently by de Gennes, the parametre $\beta$ has more fundamental value than $f$ from the point view of thermodynamics and physical kinetics: the matter is that here we witness the theory of dissipative structures and Prigozhin's bifurcation on reaching some critical value $\beta^{*}$. Bifurcation specifically means transition from a stationary decision to a non-stationary one, for example, as in case of turbulence occurrence in the general problem on flow. And it is absolutely unimportant, in what way $\beta^{*}$ is reached, even an isolated macromolecule loses stability in relation to distribution of rotatory isomers and becomes uncoiled (i.e. gets trans-conformation).

\footnotetext{
${ }^{*}$ Hereinafter we deliberately abstain from references to information sources as all of them appear in the bibliographic list.
} 
Hence, if in a system of flexible-chain macromolecules somehow, for example, under the influence of a flow with stretching there have been reached super-critical values $\beta>\beta^{*}$, then in solutions of crystallizing polymers there can appear super-molecular formations according to nucleation mechanism, and in solutions of non-crystallizing polymers according to spinodal mechanism. In terms of Prigozhin's theory at $\beta^{*}$ the system undergoes bifurcation and passes from thermodynamic to non-thermodynamic branch where super-molecular formations acquire steady form.

The above said allowed to offer a new approach to the nature of nonlinearity of filtration flows of polymer solutions. The deviation mechanism from the Darsi's law consists in occurrence of a selfoscillatory mode of reversible processes of macromolecule uncoiling under effect of quasi-regular longitudinal velosity gradients in a porous medium and influence of uncoiling macromolecular chains both on molecular and supermolecular levels on the structure of a filtration flow, i.e. as a result of oscillations of deformations of macromolecules and dynamic supermolecular structures, formed under the influence of stretching flows, there occurs higher energy dissipation and nonlinearity of a filtration flow.

Nonlinearity in a filtration flow of polymer solutions ensures alignment of front for advancement and filling layered non-uniform and cracked-porous beds with a polymer liquid, and consequently, provides a wide coverage of a bed.

The designed approach to explain the mechanism of filtration nonlinearity in polymer solutions flow well keeps within the general scheme of self-regulated processes where negative feedback dominates. It is characteristic of systems, capable to change their properties under the influence of external physical effects, in this case under the influence of longitudinal velocity gradients locally arising in pores, i.e. flows with stretching.

Nonlinearity observed at polymer solutions flow through porous media does not allow to use the linear law of filtration - Darcy's law to forecast technological indicators of feeding polymer solution in an underground layer.

Such project studies are in need of a filtration law that would describe the flow of both Newtonian and non-Newtonian fluids with 
difficult rheology in conditions of flow with stretching. In monography [2] gained a generalised filtration law to be true for polymer solutions and common Newtonian fluids. The generalised filtration law takes into account manifestation of elastic deformation effects at $\dot{\varepsilon}>\dot{\varepsilon}_{\text {кр }}$ and, hence, is true both for shift flows and for polymer solution flows with stretching.

Everything said allows to carry out complete analysis of the problems related to "abnorma" effects, arising under conditions of polymer solution flows with stretching and their use when designing corresponding of technologies of production oil and gas.

The approach designed to explain the mechanism of filtration flow nonlinearity allowed to substantiate technological variants of filling various porous media with polymer solutions which was assumed as a basis of technologyfor increasing oil production based on oil ejection from layers with polymer solutions, as well as to work out a number of other technologies. Such technologies may serve - to increase performance efficiency and reduce power consumption in various hydraulic systems - to extinguish fire with water, to supply heat, to pump out water emergently, in hydroacoustic stations of fishery vessels and other as well as in perforation of oil and gas wells by high-velocity jet of polymer solution.

Physical-chemical and technological problems are closely bound in this work, but nevertheless the technological problem somewhat dominates over the physical-chemical one. Have studied reactions of polymer-containing systems to hydrodynamic effects not with methods of phenomenological rheology but from positions of molecular hydrodynamics. It allowed them to solve a number of special problems generated by rather difficult rheology of polymer solutions under flows with stretching. In this connection, the data presented in this work, on macromolecule dynamics in conditions of a converging flow are of separate interest as well, when the polymer solution flow in the porous-fractured reservoir.

The logics of work - from fundamental research to concrete technological process. The complex approach to studying polymer solutions reaction to hydrodynamic effects allowed to predict, and further to confirm experimentally the fact of hydrodynamic field influence on floculating ability of flexible-chain linear macromolecules. This 
new phenomenon, perspective in respect of working out and upgrading technologies of cleaning various liquids the oil and gas industry, is given a proper attention inother other works [2,4,5]. There has been offered mechanism of the given phenomenon based on conceptions about strong deformation influence of a hydrodynamic field on molecular coils.

Theoretically predicted and experimentally discovered fact of longitudinal hydrodynamic field influence on floculating ability of molecules of a linear flexible-chain polymer opens a possibility to design devices allowing to increase efficiency of floculation cleaning of liquids. This allowed to offer technical decisions to realise a method of hydrodynamic activation of polymer floculants and equipment to measure residual concentration of a polymer floculant in liquids after their clarification. This is a source of prospects to intensify cleaning processes for various liquids (sewage drinking water, lubricant oils, etc.) when using polymer floculants.

\section{Displacement of Oil from Porous Bed by the Oscillating Flow of Polymer Solution}

Experiments were conducted with the aim of elucidating the nature of the mechanism of an "anomalously" high degree displacement of oil from porous bed using the polymer solution. The most contradictory opinions as to the nonlinearity of filtration flow of polymer solutions are being stated; most researchers believe that the nonlinearity is caused by the adsorption of macromolecules onto the porousmedium walls [6-8]. There is an opinion that the deviations from Darcy's law are due to the elastic-strain effects manifesting themselves in the case of flow of solutions of flexible-chain polymers in a porous medium [2,9-10]. Below, we will be dealing with experiments on checking the mechanism of an "anomalously" high degree displacement of oil from porous bed using the polymer solutions; the basis for this mechanism is provided by the concepts of a strong strain action of the hydrodynamic field on coiled macromolecules in filtration flow [11].

The mechanism of deviations from Darcy's law lies in the selfoscillating regime of reversible processes of unrolling of macromolecules under the action of the longitudinal velocity gradients quasiregularly arising in the porous medium and in the influence of un- 
rolling macromolecules at both the molecular and supermolecular levels on the structure of the filtration flow. The oscillation of the strains of macromolecules and dynamic supermolecular formations arising under the action of stretching flows results in the higher-thanaverage energy dissipation filtration and the flow nonlinearity [2]. The latter ensures the equalization of the front of advance and filling of inhomogeneous laminar beds with polymer fluid and hence a wider coverage of the bed and hence a high degree displacement of oil.

From the above mechanism of nonlinearity of the filtration of polymer solutions and from the theory of elastic-strain effects in the case of flow with stretching of polymer solutions [9,10], it follows that the higher degree displacement of oil from porous bed must be attained in oscillating injection of a polymer solution into it. To substantiate this prediction we studied the aqueous solutions of polyethylene oxide (PEO) under the conditions of oscillating hydrodynamic field with stretching.

In realizing the model regime of injection of a solution polymer into a porous medium (bed), the solution under study was forced through a flow chamber (module) under the action of a constant pressure difference (see fig. 1.1). This is an unusual kind of viscosimeter in which the hydroresistors are short capillaries.

In the stationary regime, flow through the hydroresistors was carried out by creation of a pressure difference at the viscosimeter inlet and outlet, whereas in the oscillating regime, it was carried out by superposition of a constant pressure difference and the pulsating pressure arising in the vessel between the capillaries due to the vibrations of the membrane of an electromagnetic vibrator. The amplitude of membrane vibrations was monitored by a special device. It was constant at different frequencies in each run of experiments and was selected such that the rate of flow (caused by the membrane vibrations) of the polymer solution through the capillaries was lower than the flow rate due to the constant pressure difference. The oscillating component of the flow velocity in the capillaries that was caused by the pressure pulsations in the intercapillary volume was smaller than the flow velocity in the capillary due to the constant pressure difference in the viscosimeter. The flow-rate velocity of the polymer solution through the capillaries was higher than the critical value [11]. The diameter of the capillaries was $0.2 \cdot 10^{-3} \mathrm{~m}$. 


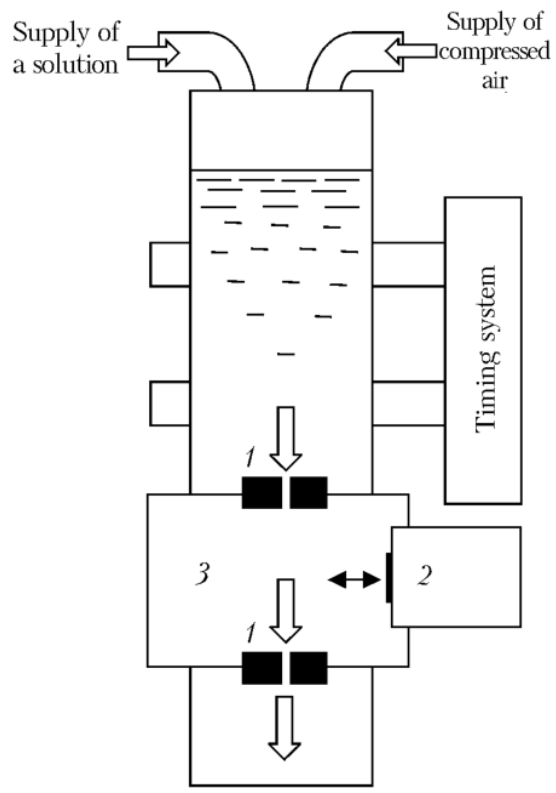

Fig. 1.1. Diagram of a viscosimetric unit: 1 - short capillary; 2 - electromagnetic vibrator; 3 - zone of action of the vibrator

The hydrodynamic efficiency of the polymer solutions under study was evaluated by the value of the efficient viscosity $\eta_{\text {ef, }}$ which was determined by comparison of the flow-rate velocities of the solution under study and calibration viscous Newtonian fluids at the same pressure difference. The entire unit was placed in a thermostat, where the temperature of the solutions under study was monitored by an electronic heat controller.

Fig.1.3 shows the experimental cell represents a layer of quartz compacted in a hermetically sealed space is filled with oil between two glass plates having inlet and outlet pipes.The inlet pipe was connected either directly to a measuring vessel, into which the aqueous polymer solution had been poured in advance, or via a module with a vibrator.The measuring vessel is equipped with a system for recording the flow-rate of the solution injected into the model of natural bed.The solution is injected due to the excess air pressure produced in the measuring vessel; the pressure is monitored by a manometer. The structure of the experimental setup enables us to visualize the process of advance of the front of the polymer solution in the porous 
bed and to qualitatively evaluate the efficiency of different regimes of oil displacement from porous bed by the flow patterns.

PEO having the viscosity-average molecular weight of $\bar{M}_{\eta}=4.5 \cdot 10^{6}$ was used as a polymeric additive in water and characteristic of a viscosity $1,72 \mathrm{~m}^{3} / \mathrm{kg}$. The aqueous solutions of polymer had concentration $C_{\mathrm{PEO}}=0.001 \div 0.050 \%$.The viscosity of the oil ranged from $10^{-2}$ to $5 \cdot 10^{-2} \mathrm{~Pa}$ sec.

The results of the experiment are presented in fig. $1.2 a$, where $\eta_{\mathrm{ef}}$ is the effective viscosity of the solution under the conditions of stationary outflow and $\Delta \eta_{\text {ef }}$ is the addition resulting under the oscillating action on the mainstream flow. The curves correspond to different velocities of mainstream flow through the capillaries. It is seen that the critical frequency of action $\omega_{\text {cr' }}$ on the stationary flow exists; beginning with this frequency, flow becomes more dissipative.

The quantity $\omega_{\text {cr' }}$ grows with average flow-rate velocity, whereas $\Delta \eta_{\mathrm{ef}}$ decreases. With lower concentrations of PEO in the solution and the same flow-rate velocity, the value of the critical frequency is higher.

When polymer systems (PEO solutions in our case) are strained, changes in the strains $\varepsilon$ and the stresses $\sigma$ occur in different phases $[12,13]$. The angle $\delta^{\prime}$ characterizing this difference is a composite function of the frequency of actions $\omega^{\prime}$. Strain in this case is carried out under the action of the stress following the law

$$
\sigma(t)=\bar{\sigma}+\sigma_{0} \exp \left(i \omega^{\prime} t\right)
$$

where $\bar{\sigma}$ - stationary tension, $t$ - time. 

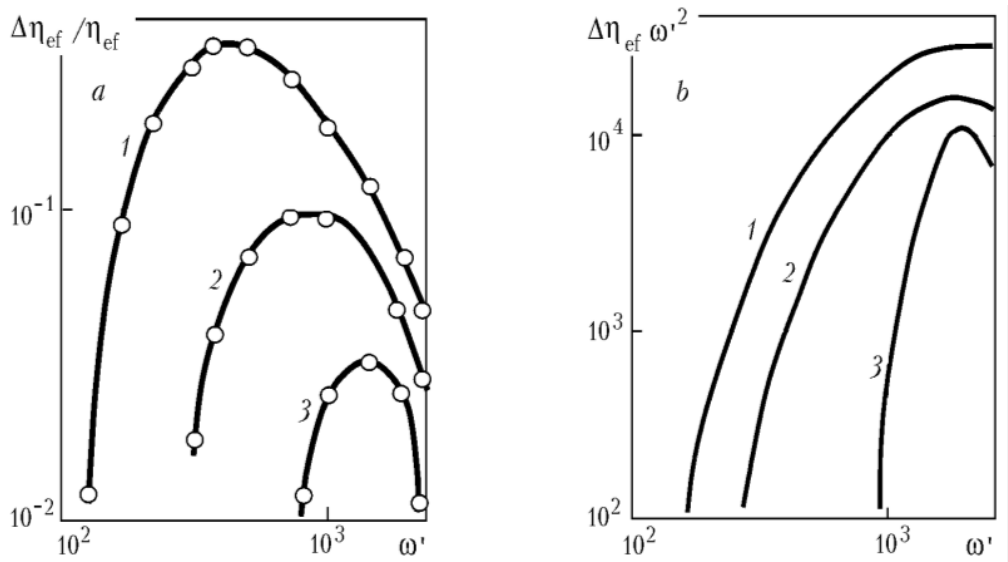

Fig. 1.2. Influence of the frequency of external action on the change in the effective viscosity (a) and the dissipative function $(b)$ in the case of flow of an aqueous PEO solution $\left(\mathrm{MPEO}=4.5 \cdot 10^{6}\right.$ and $\left.\mathrm{CPEO}=0.02 \%\right)$ through short capillaries with different average velocities: 1) 0.3 ; 2) 0.5 ; 3) $0.7 \mathrm{~m} / \mathrm{sec} . \Delta \eta_{\mathrm{ef}} \omega^{\prime 2}, \mathrm{rad}^{2} \mathrm{~Pa} / \mathrm{sec} ; \omega^{\prime}, \mathrm{rad} / \mathrm{sec}$

Taking into account that $\sigma(t)=\sigma_{0} \cos \left(\omega^{\prime} t\right)$ and $\varepsilon=\varepsilon_{0} \cos \left(\omega^{\prime} t-\delta^{\prime}\right)$, and excluding the time from these equalities we obtain the equation

$$
\left(\frac{\sigma}{\sigma_{0}}\right)^{2}+\left(\frac{\varepsilon}{\varepsilon_{0}}\right)^{2}=\varepsilon^{2} \delta^{\prime}+\left(\frac{\sigma}{\sigma_{0}}\right) \cdot\left(\frac{\varepsilon}{\varepsilon_{0}}\right) \cos \delta^{\prime}
$$

Expression (1.2) represents the equation of an ellipse whose area is equal to the work $A_{0}$ done in a cycle of harmonic vibrations and irreversibly lost (dissipating) under strain. Then the dissipation function $\mathrm{W}$ is computed as the product of $A_{0}$ and the number of cycles per unit time

$$
W=A_{0} \frac{\omega^{\prime}}{2 \pi}=\frac{\varepsilon_{0} \sigma_{0} \omega^{\prime}}{2} \sin \delta^{\prime} .
$$

From relation (1.3) it is seen that, under the same conditions of strain, the dissipative function only depends on the polymer characteristics expressed in the value of $\delta^{\prime}$. 
Changing the stresses by the harmonic law, we may track a change in the strain rate $\dot{\varepsilon}$. The latter is related to the strain $\varepsilon$ as follows

$$
\dot{\varepsilon}=\frac{d \varepsilon}{d t}=\varepsilon_{0} i \omega^{\prime} \exp \left[i\left(\omega^{\prime} t-\delta^{\prime}\right)\right]=i \omega^{\prime} \varepsilon .
$$

The value of the ratio $\sigma / \dot{\varepsilon}$ is a complex dynamic viscosity $\eta^{*}$. This quantity may be represented in the form of the real and imaginary components $\eta^{*}=\eta^{\prime} \mathrm{i} \eta^{\prime \prime}$, where $\eta^{\prime}=\left(\sigma_{0} / \varepsilon_{0} \omega^{\prime}\right) \sin \delta^{\prime}$ and $\eta^{\prime \prime}=\left(\sigma_{0}\right.$ $\left./ \varepsilon_{0} \omega^{\prime}\right) \cos \delta^{\prime}$. Introducing $\eta^{\prime}$ into relation (1.3) and performing transformations, we obtain

$$
W=\eta^{\prime} \cdot \frac{\varepsilon_{0}^{2} \omega^{\prime 2}}{2} .
$$

Dynamic viscosity manifests itself in the system under strain, and the increment of the effective viscosity $\Delta \eta_{\text {ef }}$ under the conditions of superposition of harmonic actions on mainstream flow is attributable to the appearance of $\eta^{\prime}$. Since the amplitude value of the strain $\varepsilon_{0}$ remains constant in the experiment, we may use scaling and rewrite (1.5) in the form

$$
W \approx \Delta \eta_{e f} \cdot \omega^{\prime 2} .
$$

For the experimental curves (fig. 1.2a), we have obtained dissipative-function curves (fig. 1.2b) using expression (1.6). An analysis of these curves shows that, as the average velocity of flow of the aqueous PEO solution through the capillaries increases, i.e., as the fraction of macromolecules (because of the presence of the molecularweight distribution) that have experienced unrolling $[10,11]$ and are unrolled to a higher degree, which causes the induction of rigidity in the macromolecules, increases, the dissipation of pulsating-flow energy decreases. The threshold value of the frequency, beginning from which the flow becomes distinctly dissipative, grows for less "flexible" and stretched molecules. As the induced rigidity of macromolecules increases, the dissipative curve acquires a more pronounced extremum character. 
From the data obtained it follows that oscillating filtration flow of polymer solutions must ensure a higher degree of filling and coverage of a porous bed and thus a higher degree displacement of oil from a natural bed.

Fig. 3 shows the patterns of oil displacement from of a model porous bed with a laminar in homogeneity using polymer solution. In the first version (see fig. 1.3a), displacement of oil of the porou space using polymer solutions was carried out in the stationary regime, whereas in the second version (see fig. 1.3c), it was carried out in the pulsating regime.

The structure of the experimental setup enables us to visualize the process of advance of the front of the polymer solution in the porous bed and to qualitatively evaluate the efficiency of different regimes of oil displacement from porous bed by the flow patterns.

Calculation of the injection regimes is based on the use of the Debora $(D e)$ number characterizing the beginning of the development of a higher-than-average hydrodynamic resistance: $D \mathrm{e}_{\mathrm{cr}}=\theta_{\mathrm{c}} \dot{\varepsilon}$, where $\theta_{\mathrm{c}}$, relaxation time of the solution; $\dot{\varepsilon}$, strain rate.

The relaxation time $\left(\theta_{c}\right)$ may be determined experimentally or from the known molecular characteristics of the polymer $[14,15]$

$$
\begin{array}{r}
\theta_{c}=\theta_{0} \cdot \exp (k), k<1 ; \\
\theta_{c}=\theta_{0} \cdot\left[\exp \left(k^{2 / 3}\right)\right] / k^{1 / 3}, k>1, \\
k=[\eta]_{0} \cdot C,
\end{array}
$$

where $\theta_{0}$ - relaxation time of weakly strained coiled macromolecules, $k$ - criterion of the degree of concentration of the solution, $[\eta]_{0}$ characteristic viscosity, $C$ - concentration of the polymer in the solution. 

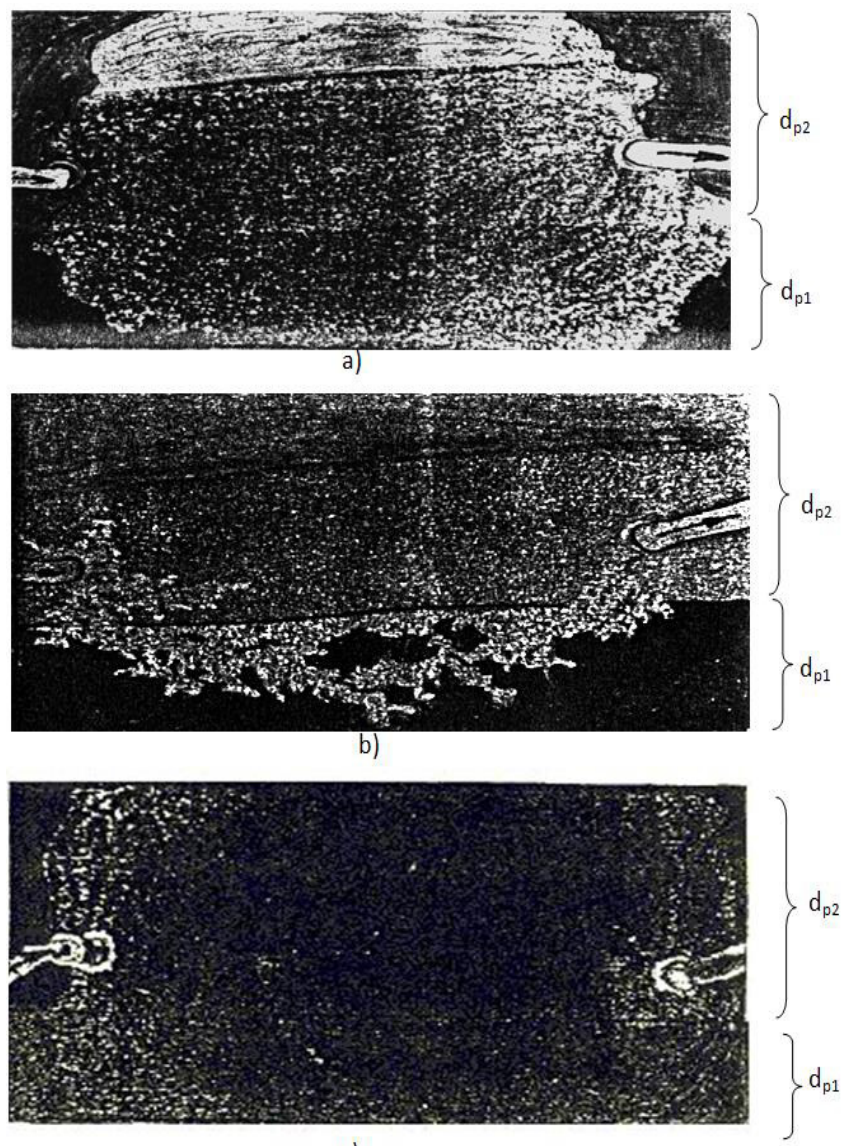

c)

Fig. 1.3. Patterns of oil displacement from porous medium with a laminar inhomogeneity $\left(d_{p} / d_{\mathrm{p} 2}=5\right)$ using PEO solution: $a$ - stationary regime of oil displacement at supercritical velocities; $b$ - the same, at subcritical velocities; c supercritical regime of oil displacement in the case of oscillating $(1000 \mathrm{rad} / \mathrm{sec})$ action on filtration flow

The longitudinal velocity gradient realized in the pore cross sections in flowing of the polymer solution defies an accurate calculation. However, we may evaluate it as a first approximation, solving the problem on flow of a Newtonian fluid in a convergent channel [2] as an element of a pore channel 


$$
\dot{\varepsilon} \approx 2 V_{f} /\left(K \cdot d_{p}\right),
$$

where $V_{f}$ - filtration rate, $K$ - coefficient of porosity of the medium, $d_{p}$ - average pore size.

For the ranges of PEO molecular weight and concentrations of practical importance, the Debora number may be assumed to be equal to unity for $k<1$ and to 2.5 for $k>1$ in accordance with experimental data.

Passing from the model to an actual filtration flow and on the basis of the results obtained, we may state that oscillating filtration flow of polymer solutions ensures a higher degree of coverage of the bed and thus a higher degree displacement of oil from a natural bed (fig. 1.3c) than stationary flow (fig. 1.3a) and stationary regime of oil displacement at supercritical velocities (fig. 1.3b).

The experiments conducted have confirmed the correctness of the prediction of the theory of elastic-strain effects in tensile flow of the higher degree of oil displacement from of a porous bed in the oscillating regime of injection of a solution polymer into a porous medium (bed) and hence the mechanism of deviations from Darcy's law the basis for which is provided by the concepts of a strong strain action of the hydrodynamic field on coiled macromolecules.

The effects causing the highly dissipative regime in oscillating flow of polymer solutions through porous media play an important role in optimization of the process of oil displacement from porous (especially with a laminar inhomogeneity) bed using polymer solutions. The injection of a solution polymer into a porous medium (bed) must occur in the oscillating regime, when elastic strains appear, and the frequency of pulsating action on the filtration flow must correspond to the maximum of the dissipative function $W(\omega) \approx \Delta \eta_{e f} \cdot \omega^{\prime 2}$.

\section{Perforation of oil and gas wells by a high-velocity jet of pol- ymer solution}

The method of hydro-sandblasting perforation (HSP) of oil and gas well casing strings is a fairly effective [16], but this method has significant disadvantages: in the process of HSP, the inner surfaces of the tubing, fittings and equipment used in the water-abrasive perforation fluidare subject to strong hydroabrasive wear; as a result of 
this hydroabrasion, the diameter and profile of the nozzle holesincreases, forming a perforation jet, which in turn leads to a gradual decrease in its velocity and to weaker perforation and longer total execution time; due to the hydroabrasive wear of the sandblasting machine nozzles, there is a need to periodically replace the nozzles, which causes additional costs for the nozzles, which are made by powder metallurgy of hard alloys, and due to an increase in the number of round-trip operations; there is intense wear on the pipes, meaning they need to be replaced; the abrasive sand particles penetrate deep into the rock in the channels which are formed, impairing the productivity of the formation. All this leads to more resourcesbeing necessary in the hydroperforation of oil and gas well casing strings.

The problem of developing a highly efficient, innovative method forhydroperforating oil and gas well casings can be approached by taking advantage of certain anomalies in the hydrodynamic behaviour of polymer solutions in longitudinal flow. That is, the cutting hydroabrasive jet in the inlet section of the hydroperforator nozzles must be replaced by a polymer solution jet [17]. The polymer must be a safe substance which is approved for use in the oil and gas industry. Such polymers include PEO, polyacrylamide (PAA) or hydrolysed polyacrylamide (HPAA). For example, PEO is a safe substance that is used as a flocculent to purify and disinfect drinking water, as a thickener in the food industry, in pharmaceuticals and more $[18,19]$.

An analysis of the literature data shows that modern ideas about the behaviour of macromolecules under the conditions of convergent flow - as in the entrance area of the jet-forming nozzle of a hydroperforator - are far from perfect. Currently, we are transitioning from an accumulation of experimental information to an understanding of its physicochemical essence and to establishing the basic patterns of its rubber-like, high-elasticity manifestation. New knowledge is needed to understand the rheophysics of several anomalies during the flow of polymer solutions through the nozzle of ahydroperforator, which would allow very efficient perforation of the casing strings of oil and gas wells with a high-velocity polymer solution to be developed. 
Of particular scientific and practical interest is the study of transitions, which result in structures that are far from equilibrium. Nonequilibrium thermodynamics proves that non-equilibrium phase transitions are possible. These transitions have only been thoroughly studied in concentrated polymer solutions, while the dynamic phase transitions in dilutepolymer solutions are only studied in a longitudinal field, which is created by the superposition of two coaxially located streams [20-22]. It has long been suggested that such transitions are impossible under the conditions of convergent flow of dilute polymer solutions. It was previously proved that convergent flow is a superposition of currents undertension and shear forces [23]. This is primarily due to the interest in work on the dynamics of polymer chains in convergent flows, because a strong deformation effect on macromolecular balls must take place in the inlet of the nozzle of the hydroperforator [24].

The most significant results in the study of the structural changes in polymer solutions under the action of a homogeneous longitudinal hydrodynamic field were obtained by Brestkin et al. [21]. The main result of this work is the proof that in the conditions of a homogeneous longitudinal hydrodynamic field, dynamic phase transitions are possible. However, this findingrefers to a homogeneous longitudinal field and not to convergent currents, which takes place in the inlet area of a drill nozzle. This indicates the relevance of research on the anomalous phenomenon of the high destructive capacity of a waterpolymer jet. According to the results of the review of convergent [23] and turbulent flows of polymer solutions [25] and the use of polymer additives in cutting materials [26,27], it has been established that the process of perforating oil and gas well casing strings with a stream of polymer solution requires further study. It becomes obvious that the development of a highly efficient process for hydroperforating oil and gas well casing strings can be approached using features ofthe hydrodynamic behaviour of polymer solutions observed in a longitudinal flow, which occurs in the inlet section of hydroperforator nozzles.

The aim of the study is to develop an efficient, innovative method of perforating oil and gas well casing strings with a high-velocity stream of polymer solution that conserves resources and energy and 
meets the requirements for environmentally friendly hydrocarbon production.

The perforation work was performed on laboratory stands. A laboratory hydraulic stand based on an URG-3020 unit - with an operating pressure of up to $500 \mathrm{MPa}$, had a jet diameter at the nozzle of $3 \cdot 10^{-3} \mathrm{~m}$ and a function for controlling both the integral and differential parameters of the process of material destruction by a waterpolymer jet - was used as the source of high-speed fluid flow. A model was created for the obstacle (target) to test the effectiveness of perforation: a kind of "sandwich" which contained a 10-mmthicksheet of steel - with a strength category of D according to GOST 632-80, which simulated a casing - a concrete layer, 20-mm-thick ring and a layer of rock (compressive strength: $600 \mathrm{~kg} / \mathrm{cm}^{2}$ ) with a total thickness of $0,8 \mathrm{~m}$.

We also used an industrial water jet, which provided $450 \mathrm{MPa}$ of pressure in the jet-forming nozzle, an average jet velocity of up to $350 \mathrm{~m} / \mathrm{s}$ and had a jet diameter at the nozzle of $1 \cdot 10^{-3} \mathrm{~m}$. A detailed description of the hydrodynamic part of the water jet, its characteristics andthe methods for determining the average speed, momentum and energy of the jet can be found in Atanov [27], Pogrebnyak et al. [28] or Brenner et al. [29]. To qualitatively assess the energy capabilities of the jet in the experiments, we studied the interaction between the jet and the obstacle installed on a physical pendulum. The barrier was made of steel (category of S3) sheets with dimensions of $220 \times 220 \times 3 \cdot 10^{-3} \mathrm{~m}$ with mounting holes. There was a gap of $5 \cdot 10^{-2} \mathrm{~m}$ between the plane of the obstacle and the body of the physical pendulum.

An optical-mechanical complex and a polarisation-optical installation [30] were used to study the peculiarities of the flow of polymer solutions under model conditions of jet-forming nozzles, as were the dynamics of polymer macromolecules in the inlet section of hydroperforator nozzles.

Solutions with a completely dissolved polymer were prepared by diluting to the desired concentration of a prepared $0.1 \%$ polymer solution. Because aqueous solutions of PEO (as well as other polymers) have significantly different properties during long-term storage, $0.05 \%$ potassium iodide is used as a stabilizer in aqueous solutions of PEO, which almost completely eliminates the ageing of PEO 
in water during storage and does not affect its hydrodynamic activity [23]. PEO solutions with intrinsic viscosities of 2.42 and $1.72 \mathrm{~m}^{3} / \mathrm{kg}$ and molecular weights of $6 \cdot 10^{6}$ and $4 \cdot 10^{6}$ were used.

The novelty in the design and components of this research is the comprehensive study of the reactions of polymer solutions to the hydrodynamic effect of tension, i.e. under the conditions of the jetforming nozzle of a hydroperforator. Complexity is a necessary condition for solving not only fundamental scientific problems, but also technical ones (from basic research to a specific technological process).

Establishing the nature of the destructive capacity of a polymer solution jet is important for the development of an efficient, innovative method of perforating oil and gas well casings with a high-speed polymer solution jet. Contradictory opinions have been put forward about the mechanism of the destructive power of a high-velocity jet of a polymer solution. Some researchers believe that the increased destructive capacity of the water jet when small polymersare added is caused by the Toms effect during the flow of the working fluid through the nozzle that forms the jet [26]. Based on this, it is argued that the jet with polymer additives has a higher speed and dynamic pressure than a jet of pure water at the same operating pressure and nozzle diameter, and therefore destroys more of the material. In other words, it is assumed that associates of polymer molecules are formed in a water-polymer jet (without experimental proof) [31]. In [27,32], the high destructive capacity of a high-velocity jet of a polymer solution is associated with the deformation effects that occur during the flow of aqueous solutions of polymers undertension. Therefore, we discuss the experiments that were set to clarify the peculiarities of a polymer solution jet's destructive capacity, which is based on the idea that a strong deformation of the hydrodynamic field occurs on macromolecular bundlesin the inlet of the nozzle.

This experiment was conducted using a hydraulic stand of industrial manufacture. The experimental data are presented in table 2.1. and fig. 2.1. It can be seen that the maximum distance from the jetforming nozzle of the hydrostand to the target obstacle, when the target obstacle puncture is still observed, is $1.5 \mathrm{~m}$ for water and $2.5 \mathrm{~m}$ for a $0.0008 \%$ aqueous polymer solution; the effectiveness of 
PEO additives was observed in concentrations starting from0.004\%; and that PEO impurities reduced the average jet velocity.

Table 2.1

The effect of PEO concentration and distancefrom the nozzle to the target obstacle on the interactionwith a jet of PEO solution

$\begin{array}{cccc}C_{\mathrm{PEO}}, \% & \begin{array}{c}\text { Distance to } \\ \text { the target, } \mathrm{m}\end{array} & \begin{array}{c}\text { Average velocity } \\ \text { of jet flow, } \mathrm{m} / \mathrm{s}\end{array} & \begin{array}{c}\text { Nature of target obstacle } \\ \text { Diameter of a hole } \\ (15-18) \cdot 10^{-3} \mathrm{~m}\end{array} \\ 0 & 1,5 & 255 & \begin{array}{c}\text { No puncture } \\ \text { No puncture }\end{array} \\ 0 & 1,75 & 250 & \begin{array}{c}\text { Diameter of a hole } \\ (15-18) \cdot 10^{-3} \mathrm{M}\end{array} \\ 0,0004 & 2,5 & 250 & \begin{array}{c}\text { No puncture } \\ 0,0004\end{array} \\ 0,0008 & 1,5 & 240 & \begin{array}{c}\text { Diameter of a hole } \\ (8-10) \cdot 10^{-3} \mathrm{M}\end{array}\end{array}$

The experimental data are fully explained by a strong deformation effect of the hydrodynamic field on molecular bundles of the polymer. In a previous study [33], the transition of the bundle-expanded chain at a convergent flow (in the model conditions of the nozzle of the hydroperforator) of aqueous solutions of PEO was experimentally revealed. It is shown that the hydrodynamic field, which arises under conditions of convergent flow, leads to a significant degree of expansion of polymer molecules (60-70\%) and to a bundle-to-chain transition.
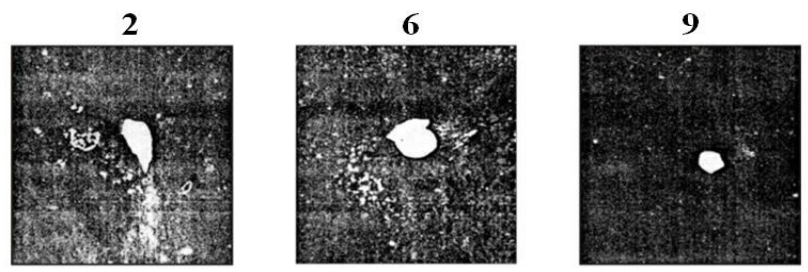

Fig. 2.1. Photos of the target obstacle after interaction with jets of water and an aqueous solution of PEO: $2-$ water; $6-M_{\mathrm{PEO}}=6 \cdot 10^{6}, C_{\mathrm{PEO}}=$ $0,0004 \% ; 9-M_{\mathrm{PEO}}=6 \cdot 10^{6}, C_{\mathrm{PEO}}=0,0008 \%$ 
The longitudinal velocity gradient, which occurs in the contraction cone nozzle of the hydrostand, can be calculated as not exceeding $2 \cdot 10^{3} \mathrm{c}^{-1}$. Therefore, despite the high speeds $(250 \mathrm{~m} / \mathrm{s})$ and resulting large shear velocity gradients, the conditions of deformation of polymer macromolecules in the conical nozzle of the water jet and in the inlet section of the model nozzle are almost the same. This indicates that during the flow of an aqueous polymer solution in the nozzle of the water jet, the PEO macromoleculesare subjected to strong deformation of the longitudinal hydrodynamic field. All this supports the claim that the high-velocity water-polymer jet is "reinforced" by highly developed macromolecular chains of the polymer, and - considering the results from Pogrebnyak et al. [34] - then supramolecular structures. Part of the energy goes to the structural adjustment of the flow [17,33], which lowers the average flow rate of the aqueous polymer solution and improves the quality of its formation, asa jet with small polymer additives is more compact (has a smaller diameter) than water (see fig. 2.1 and table 2.1). This contributes to an increase in the maximum distance from which the destruction of the steel barrier/target can occur.

The data of the experiments which are presented in table 2.1 convincingly testify that the high destructive capacity of a high-velocity water jet with small polymer additives is not due to the Toms effect. This is confirmed, first of all, by the $8-10 \%$ reduction in the average jet velocity of the aqueous PEO solution relative to the water jet's velocity at the same operating pressure and nozzle diameter. Thus, despite the lower flow rate of the polymer solution, its destructive capacity was much higher than that of the water jet.

The conclusion that the large destructive capacity of the highvelocity water jet with dissolved small polymer impurities was not due to the Toms effect also follows from the analysis of turbulent fluid flow presented below. In the case of near-wall turbulence, its structure should be affected by highly uncoiled macromolecular chains $[1,35,36]$. To do this, there must be certain conditions in the turbulent flow, namely, the longitudinal velocity gradients and the time of deformation in them must be greater than $0,5 / \theta_{\mathrm{c}}$ and $\theta_{\mathrm{s}}$, accordingly (where $\theta_{\mathrm{c}}$ is the relaxation time of the polymer solution). At some fairly high dynamic velocities, when the formation and de- 
struction of tensile zones occur at a high frequency, as follows from the work by Cantwell [37], increasing the strain rate should reduce the strain factor by increasing the strain rate should reduce the strain factor by decreasing the time of the longitudinal velocity gradient. This is due to small-scale turbulence, the percentage of which increases sharply at high velocities [37,38]. Also, the degradation of polymer solutions significantly reduces the Toms effect at high velocities [23]. Therefore, in high-velocity turbulent flows, the hydrodynamic activity of PEO should decrease due to the discrepancy between the characteristic relaxation times of the aqueous PEO solution, the time scale of the flow (emissions) and the degradation of the PEO macromolecules.

To confirm the correctness of the above approach, it is necessary to conduct a detailed study of the polymer solution's flow through the nozzles of a hydroperforator. At the same time, there are obvious advantages of using currents in the model of nozzles forming a stream in order to research the macromolecules' interaction with a hydrodynamic field in connection with thepossibility of studying experimentally the features of a polymer solution stream formingunder controlled conditions.

Before proceeding to the discussion of the experimental data, let us consider the parameters that will characterise the dissipative processes in flow model systems of jet-forming nozzles.The energy dissipated per unit of time during the flow of a liquid is equal to

$$
W=\Delta P \cdot q \text {, }
$$

where $\Delta P$ is the pressure drop in the system or in its individual sections at a given flow rate $q$. This is the work that must be spent per unit of time to push the liquid through the jet-forming nozzle. Equation (2.1) is valid for any type of flow (shear, longitudinal or the superposition of one and the other - convergent) and any shape of jetforming nozzle. Energy dissipation can be represented as

$$
W=\frac{\eta e f \cdot q^{2}}{k_{f s}},
$$

where $k_{f s}$ is the constant of the flowing system and $\eta_{e f}$ is theeffective viscosity which characterises the resistance of the continuous envi- 
ronment to irreversible changesin form at various sites ina current and is calculated by expression

$$
\eta_{e f}=k \frac{\Delta P}{f_{s}} .
$$

The constant $k_{f s}$ is determined experimentally by using Newtonian fluids with known viscosities.

From Equation (2.2) it follows that dissipation at a liquid stream can be characterised using effective viscosity. For a Newtonian fluid, it always coincides with the shear viscosity, whereas the effective viscosity of a non-Newtonian medium depends on the flow regimes and is an average characteristic. The latter is due to the fact that the viscosity of such a medium is different at points in space with different rates of deformation [39].

The data that characterise the influence of outflow velocity through a nozzle on effective the viscosity of PEO aqueous solutions of various concentrations for molecular weight $6 \cdot 10^{6}$ are given in fig. 2.2.

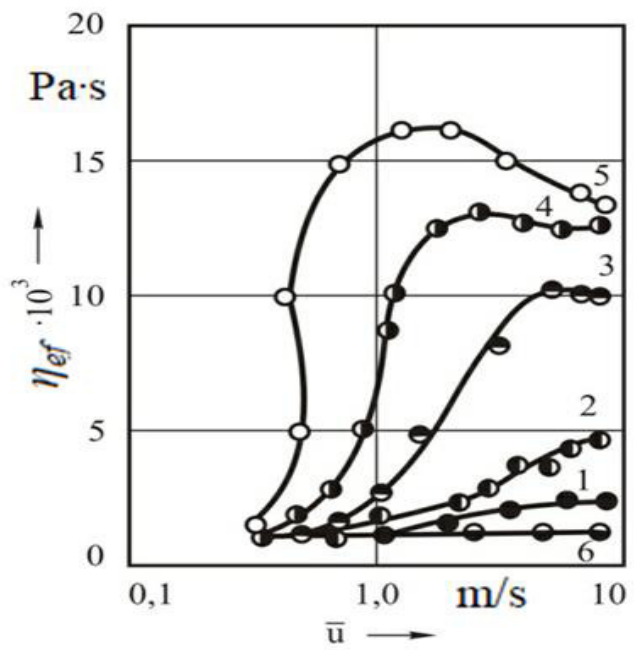

Fig. 2.2. Dependence of an aqueous $P E O$ solution's effective viscosity on its average flow through a nozzle with a square inlet $\left(d_{\mathrm{noz}}=0.12 \cdot 10^{-3} \mathrm{~m} ; \mathrm{M}_{\mathrm{PEO}}=6 \cdot 10^{6} ; \mathrm{C}_{\mathrm{PEO}}\right.$ : $1-0.0005 \% ; 2-0.001 \% ; 3-0.002 \% ; 4-0.003 \% ; 5-0.004 \% ; 6$ - water) 
The error of viscosity measurement did not exceed $2 \%$. It is seen that at relatively low velocities, the solutions behave like Newtonian fluids and their viscosities are close to the viscosity of the solvent (water). At the critical threshold value, $\bar{u}=u_{c r}$, the viscosity of the solution begins to increase; the sharper the increase, the higher the concentration of the polymer. The absence of growth of effective water viscosity with growth suggests that the specified effect is not connected with inertial turbulence. The data describing the influence of the diameter of the nozzle's aperture on the effective viscosity of PEO solutions show (fig. 2.3) that increasing the diameter of the nozzle essentially increases the critical speed.

These experimental results (fig. 2.3) allow us to assume that the observed changes of viscosity with growth $\bar{u}$ due to first of all at the expense of a difficult structure of the hydrodynamic field at the entrance section of the jet-forming nozzle (not at the expense of the macromolecules' adsorption). Studies of solutions in a stationary Kuett field have shown that they can detect a decrease in viscosity only with increasing velocity gradient [40] and only in turbulent motion do they show certain anomalies (Pilipenko, 1980). Therefore, the change in the geometry in the inlet walls of the jet-forming nozzles (i.e. the conditions of solution deformation) should change the nature of the dependence of $\eta_{e f}$ on $\bar{u}$.

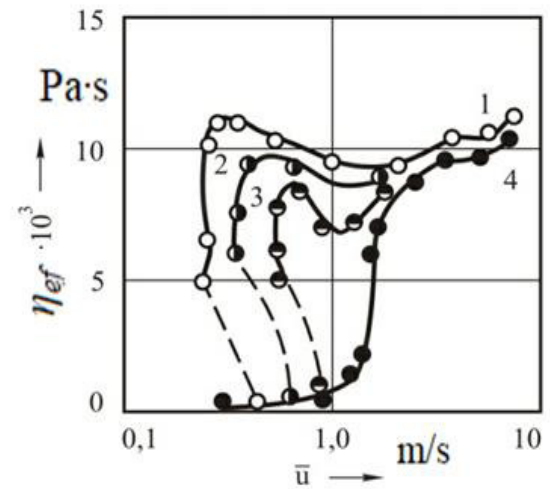

Fig. 2.3. Theinfluence of the nozzle diameter on the effective viscosity of PEO aqueous solutions $\left(\mathrm{C}_{\mathrm{PEO}}=0.02 \%\right.$; $\mathrm{MPEO}_{\mathrm{PEO}}=4 \cdot 10^{6} ; \mathrm{d}_{\mathrm{noz}}: 1-0.34 \cdot 10^{-3} \mathrm{~m} ; 2-0,42 \cdot 10^{-3}$ $\left.\mathrm{m} ; 3-0.52 \cdot 10^{-3} \mathrm{~m} ; 4-0,6 \cdot 10^{-3} \mathrm{~m}\right)$ 
Fig. 2.4 shows the curves that reflect the influence of conditions (inlet angle) of the polymer solution's flow through the jet-forming nozzle with an inlet angle of $180^{\circ}$ and $45^{\circ}$ (smooth inlet and a standard factory nozzle) on the effective viscosity. It can be seen that the flow of the PEO solution in the first case (curve 1) is highly dissipative, while in the second (curve 2) it is slightly dissipative. At the same time, for a Newtonian liquid (water), the angle of entry into the nozzles does not play a significant role. A similar conclusion follows from the analysis of the data in fig. 2.5, which shows the results of experiments to identify the effect of the angle of entry into the nozzle and the PEO concentration on the effective viscosity of polymer solutions. The experimental data characterise the peculiarities of the flow of PEO aqueous solutions through the model nozzles of a hydroperforator.

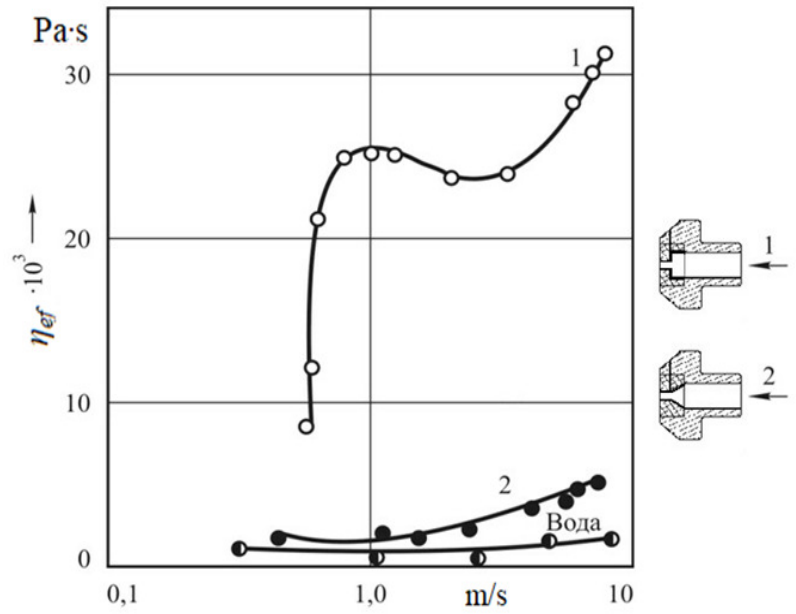

Fig. 2.4. The influence of conditions of the entry into the nozzle on effective viscosity: $\mathrm{MPEO}_{\mathrm{PEO}}=6 \cdot 10^{6}, \mathrm{C}_{\mathrm{PEO}}=0,1 \% ; d_{\min }=0,53 \cdot 10^{-3} \mathrm{~m} ; 1$ - rectangular entry $\left(180^{\circ}\right), 2$ - smooth entry $\left(45^{\circ}\right)$

From the experiments regarding the detection of the influence of the entrance angle into a nozzle on the effective viscosity of PEO solutions, it is clear that there are phenomena that are typical for such flows, yet not for purely viscous environments. At some critical values of $u_{k p}$, the effective viscosity begins to increase sharply, and the 
sharper this increase, the higher the concentration of polymer in solution and the wider the angle of entry into the nozzle. The highest value for $\eta_{e f}$ was observed for a nozzle with an inlet angle of $180^{\circ}$, i.e. when there was a sudden narrowing. Reducing the angle of entry into the hole of the nozzle leads to a decrease in $\eta_{e f}$ and displacement of the critical (threshold) speed in the direction of large values.

The ambiguity of the effective viscosity's dependence on $\bar{u}$ (one velocity can correspond to two values of viscosity, see figs. 2.3 and 2.5) testifies to the difficult nature of these currents and, naturally, studying only integral characteristics of a flows draws attention not enough to understand the observed phenomena. As mentioned above, in a previous study [33] the transition of the bundle-unfolded chain at a convergent flow (in the model conditions of the hydroperforator nozzle) of aqueous solutions of PEO was experimentally revealed.

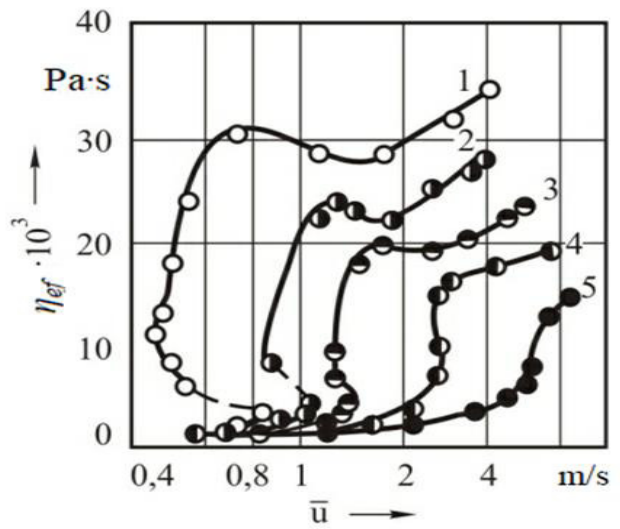

Fig. 2.5. The influence of the inlet angle on the effective viscosity when PEO aqueous solutions flow through the nozzles: $\mathrm{MPEO}_{\mathrm{PEO}}=6 \cdot 10^{6}, \mathrm{CPEO}_{\mathrm{PEO}}=0,1 \%, \beta: 1-$ $180^{\circ}, 2-150^{\circ}, 3-98^{\circ}, 4-65^{\circ}, 5-35^{\circ}$

It has been established that the water-PEO system at convergent flow under certain regimes is self-regulating with negative feedback. It has been shown that increasing longitudinal velocity gradients leads to deformation of the macromolecular coils, which in turn causes a rearrangement of the flow structure and increase the time of influence of the longitudinal velocity gradient on the macromolecular chains. The hydrodynamic field, which occurs under conditions of 
convergent flow, leads to a significant proportion of the polymer molecules unfolding, hence the peculiarities of the convergent flow of even dilute solutions due to the ability of the macromolecules to easily change the shape of these molecular balls under the action of the complex structure of the hydrodynamic field that arises in the inlet nozzle of the hydroperforator and due to the increased molecular interactions at the molecular-supramolecular level caused by these changes. Therefore, experiments proving the possibility of supramolecular structure formation during the flow of an aqueous solution of PEO in a model of jet-forming nozzles of a hydroperforator are fundamental in terms of solving the destroying of nature, which causes the destructive power of a high-speed water-polymer jet. Below we turn to such experiments.

Experimental studies [17] have demonstrated that selforganisation in space and time can be observed in aqueous solutions of PEO in the inlet section of the nozzle, which forms a jet (flow undertension). Therefore, to create a hydrodynamic field under tension, a convergent flow of water into a small hole in the plate (a model of a nozzle) with a diameterof $3 \cdot 10^{-4} \mathrm{~m}$ was used. Streams of PEO solutionwith a molecular weight of $6 \cdot 10^{6}$ were introduced into the water stream at a sufficient distance from the hole. The range of concentrations studied was $0.001 \%-0.1 \%$. The speed of the aqueous solution of PEO at the points of introduction of jets coincided with the speed of the main flow of water. Visualisation of the flow in the inlet area of the hole was carried out using admixtures of dye in the injected aqueous solutions.

At low flow rates, the coloured streams of PEO aqueous solution visualised the flow lines of the main stream (fig. 2.6). The behaviour of the streams of PEO solutions was no different from the behaviour of streams of pure water injected into the convergent stream. When a critical water flow through the outlet of the nozzle was reached, the nature of the flow of jets of aqueous solution changed dramatically. Quite thick streams of PEO aqueous solution were transformed into thin threads, the length of which changed over time (fig. 2.7). When observing the dynamics of the formation and destruction of individual threads (this is the oscillating nature of the movement of flooded streams of PEO solution) the following pattern is revealed. Initially, 
as the jets of PEO solution approached the hole, they were observed to bend smoothly towards the hole. At the same time, their velocity along these curvilinear trajectories began to increase more and more noticeably, as evidenced by the decrease in the thickness of the streams. Near the hole there was an abrupt decrease in the thickness of the streams of aqueous PEO solution, and they were transformed into a thin thread. The area of transformation of the thick stream into a thin thread began to move up the streams, which led to an increase in the length of the threads. Since the streams of the aqueous solution of PEO approached the hole at the same time, at each time the length of the threads spun by the flow from the individual streams of aqueous solution was different.

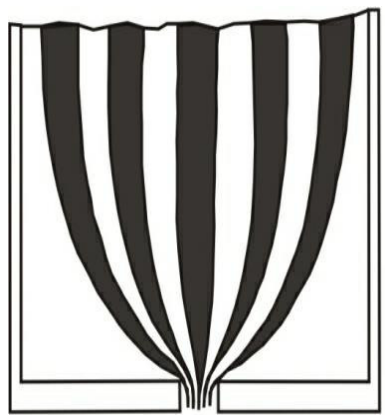

Fig. 2.6. The flow in the inlet section of themodel nozzle when the PEO aqueous solutions were injected into water (МРEO $=6 \cdot 10^{6} ;$ СРеO $=0,03 \% ; \bar{u}<u_{c r}$ )

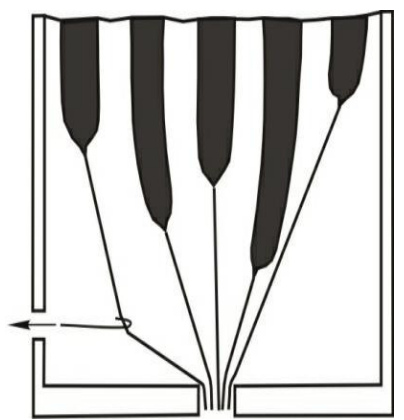

Fig. 2.7. The flow at the moment of wire probe effecting a polymer thread spun by hydrodynamic field $\left(\mathrm{MPEO}_{\mathrm{PE}}=6 \cdot 10^{6}\right.$; $\mathrm{CPEO}=0,03 \% ; \bar{u}=2,5 \mathrm{~m} / \mathrm{s}, \bar{u}>u_{c r}$ )

When acritical length was reached, the thread in the immediate vicinity of the hole broke. After that, the thread remaining at the top lost elasticity and sagged, i.e. one of the lines of the main flow of water. When the stream of aqueous PEO solution again approached the hole, everything repeated. The observed behaviour of flooded streams of aqueous PEO solution during tensile flow is due to the development of many PEO balls under the action of a hydrodynamic field. Rolling out molecular chains of PEO led to a decrease in their flexibility, which under certain conditions caused phase separation under the action of a hydrodynamic field, i.e. leading to the formation of a dynamic supramolecular structure. 
The following experiment can confirm this formation of a dynamic supramolecular structure under the action of a tensile hydrodynamic field. In the subcritical modes of main water flow leakage in the inlet area of the hole, a thin wire-probe with a device for capturing the thread of an aqueous solution of PEO was introduced. The movement of this probe wire in a plane perpendicular to the jets did not change the flow pattern, asthe jets of the aqueous PEO solution in these flow regimes are permeable to it. If the same experiment is repeated in the supercritical mode of water leakage, it is possible to capture one or more a polymer threads at the time of their growth and move aside, as shown in fig. 2.7. This is only possible in the presence of a sufficiently strong interaction between PEO molecules, i.e. in the emergence of a supramolecular structure, which is formed in this case under the action of a tensile hydrodynamic field. The minimum concentration of aqueous PEO solutions, when it was still possible to divert the aqueous solution of PEO, was $0.008 \%$, which corresponds to the region of dilute solutions when the intrinsic viscosity $[\eta]_{0}$ multiplied by the concentration of PEO $C_{\mathrm{PEO}}$ was less than 1 [40].

These results and those given above suggest that aqueous solutions of PEO can be divided into at least three regions of concentration. This is the region of dilute aqueous PEO solutions, where PEO macromolecules under the action of a hydrodynamic field are subjected to strong deformation effects (expansion of macromolecules), but still weakly interact with each other in the expanded state. The second is the region of semi-dilute aqueous PEO solutions (intermediate), when in subcritical flow regimes the solutions are diluted, and in the supercritical ones - already concentrated in the result of the formation of supramolecular structures under the action of a hydrodynamic field. These dynamic structures should be considered the type of dynamic phase transitions dealt with by the theory of dissipative structures [42]. The last region is the region of concentrated aqueous solutions of PEO $\left([\eta]_{0} \cdot C_{P E O}>1\right)$, when significant interactions between PEO molecules exist without the action of a hydrodynamic field. It should be noted that the first two areas relate to the concentrations of the manifestation of great destructive power of a high-velocity jet of an aqueous solution of PEO. Thus, when aqueous 
solutions of PEO flow through the nozzles, forming a high-velocity jet, there may be a dynamic supramolecular structure that is subject to Prigogine's principles of self-organisation [42]. These results are crucial for elucidating the nature of the great destructive capacity of a high-velocity water-polymer jet.

By elucidating the dynamics of macromolecules in the hydrodynamic field of a jet-forming nozzle and experimentally proving the formation of a dynamic supramolecular structure in the inlet section of the nozzle, we can obtain reasonable ideas to explain the mechanism behind the destructive capacity of a water-polymer jet.

Analysis of the concentration dependence of the destructive capacity of polymer solutions and the results indicating on formation of dynamic supramolecular structures in polymer solutions suggest that approaching the optimal concentration of polymer $\left(\mathrm{C} \geq \mathrm{C}_{\mathrm{opt}}\right)$, the destructive capacity of water generates anisotropic supramolecular formations that have a lifetime 10 times longer than the relaxation time of the macromolecule [33]. A further increase in the PEO concentration led to the creation of conditions for the interaction between individual molecules of PEO, even without the action of a hydrodynamic field. This leads to a sharp increase in the usual viscosity and, of course, to a relatively strong decrease in the flow rate of the aqueous solution. The consequence of this is a decrease in the destructive capacity of the water-polymer jet when reaching a relatively high concentration of PEO in aqueous solution.

To substantiate and systematise the physical laws of the fracture mechanism in the process of perforating the casing strings of oil and gas wells with a stream of polymer solution and the relationship of the individual phenomena that occur, it is important to develop schemes for calculating basic technological parameters and rational modes of a hydroperforator. The experimental data show that the process of water-polymer perforation of oil and gas well casing strings is complex and has specific features which contrast with the perforation of the water jet and hydroabrasive jet.

The subject of the work - to develop a highly efficient innovative method for perforating oil and gas well casing strings - can be achieved through a method of hydrostream perforation of the well that includes directing the jet to the place of perforation, sealing the inner cavity of the tubing and jet apparatus, actuating the ball valve located at the bottom of the jet apparatus, sealing the annulus with a self-sealing gland, and supplying the tubing pipe with cutting fluid, that is, an aqueous solution of PEO with a molecular 
weight of $M=6 \cdot 10^{6}$ at a concentration of $C_{\mathrm{PEO}}=0.003-0.007 \%$ by weight and with an operating pressure of $100-300 \mathrm{MPa}$. These parameters are the innovative technological feature in the function of this cutting fluid.

Perforation works were performed in the laboratory using a hydraulic stand based on a URG-3020 installation. The model of the obstacle (target) on which the perforation efficiency was tested is described in the experimental part. The results of the destruction of the model barrier by a waterpolymer jet with PEO concentrations of $0.0025-0.008 \%$ and a PEO molecular weight of $6 \cdot 10^{6}$, a leakage pressure of $200 \mathrm{MPa}$, a nozzle diameter of $3 \cdot 10^{-3} \mathrm{~m}$ and a perforation time of $10 \mathrm{~min}$ are provided in table 2.2.

Table 2.2

The effect of PEO concentration on the length of the channel formed in the model obstacle

\begin{tabular}{c|c|c}
\hline No of research & CPEO, $\%$ & $\begin{array}{c}\text { Length of formed channel, } \\
10^{-3} \mathrm{~m}\end{array}$ \\
\hline 1 & 0,0025 & 0,245 \\
\hline 2 & 0,003 & 0,385 \\
\hline 3 & 0,005 & 0,405 \\
\hline 5 & 0,007 & 0,430 \\
\hline 5 & 0,008 & 0,435 \\
\hline
\end{tabular}

The tests were also performed with a concentration of PEO aqueous solution of $0.005 \%$, but the pressure of the destructive fluid changed. These results are presented in table 2.3.

Table 2.3

The effect of the pressure of the destructive fluid on the length of the channel formed in the model barrier with a formed $0.005 \%$ concentration of PEO in the water-polymer stream

\begin{tabular}{c|c|c}
\hline No of research & $\begin{array}{c}\text { Pressure of cutting fluid, } \\
\text { MPa }\end{array}$ & $\begin{array}{c}\text { Length of formed channel, } \\
10^{-3} \mathrm{~m}\end{array}$ \\
\hline 6 & 75 & 225 \\
\hline 7 & 100 & 380 \\
\hline 8 & 200 & 405 \\
\hline 9 & 300 & 420 \\
\hline 10 & 350 & 425 \\
\hline
\end{tabular}

Decreasing the pressure below $100 \mathrm{MPa}$ reduced the perforation efficiency, while raising it above $300 \mathrm{MPa}$ caused a slight increase in the length of the channel; however, in a production environment there are technical difficulties in maintaining such high pressures. 
Comparative perforation works were performed on a similar model of the obstacle using HSP (sand fractional composition: 0.5-1.2 $10^{-3}$ $\mathrm{m}$; concentration: $30-50 \mathrm{~kg} / \mathrm{m}^{3}$; leakage pressure: $200 \mathrm{MPa}$. In the case of HSP, the length of the channel in the obstacle model was $320 \cdot 10^{-3} \mathrm{~m}$.

The results presented in tables 2.2 and 2.3 show that PEO additives in the selected range of concentration and flow regimes will lead to greater channel formation efficiency during the perforation of oil and gas well casings, which will allow more efficient use of oil and gas fields' potential.

Experimental and field testing of the proposed method of perforatingthe casing columns of oil and gas well casing strings- using a jet of PEO aqueous solution - which was carried out during the secondary opening of the productive reservoir at a well in the Carpathian oil-andgas-bearing region, confirmed the practical and economic feasibility of its use. The environmental friendliness of PEO additives should be noted, because this polymer is not harmful to humans and does not harm nature.

The mechanism behind the destructive power of a high-velocity jet of polymer solution has been discovered. It is not due to the Toms effect, but consists in the destructive action of the dynamic pressure of the water-polymer jet, "reinforced" by highly developed macromolecular chains and dynamic anisotropic supramolecular structures formed under the action of expanding flow in the inlet section of the hydroperforator nozzle that forms the high-velocity jet flow.

Research and field testing of the method of hydrojet perforation of well casingsthat uses aqueous solutions of PEO confirmed that the process of well perforation is more efficient, environmentally friendly and resource-saving than hydro-sandblasting perforation.

The new data on the influence that different jet-forming nozzles have on the hydrodynamic behaviour of aqueous polymer solutions during their flow through the nozzles and the established patterns of the influence that the structures have on the efficiency of well perforation - aimed at developing a highly efficient process for perforating oil and gas well casing strings with a high-velocity stream of polymer solution - are innovative, relevant and promising.

3. The nature of Hydrodynamic Drag Reduction of Oil Flow in Pipelines by Polymer Additions 
The problem of reducing the energy intensity of the oil-trunk pipelines by increasing their productivity remains one of the key problems of economic development of different countries. It is obvious that the task of improving the hydrodynamic characteristics of the oil pipelines is to find the ways to reduce the oil flow drag - fluid friction. Among the known methods of artificial influence on the boundary layer, in order to reduce the hydrodynamic drag in oil pipelines, special place is taken by a method, based on the injection of polymer solutions. This method is the only one in the development that has certain practical progress [43-46].

Laboratory and field tests of the influence of small polymer additions to reduce the drag of oil flow in pipelines began conducting since the late 70 -ies of the XX century [45,47-50]. The design development of oil pipeline by using polymeric additions for turbulent drag reduction was justified, because the actual increase of the TransAlaska oil-trunk pipeline capacity [44] has amounted 20\%. However, the resulting friction drag reduction effects by applying polymer solutions in oil pipelines are far from the theoretically predicted. Therefore, the technical task of reducing the hydrodynamic resistance of oil flow in the pipelines by applying the polymer solution into the boundary layer needs to be resolved.

This applies to all questions above regarding the nature of turbulent drag reduction by using polymer additions. Understanding the nature of reducing drag flow of oil in pipelines by small polymer additions will allow to develop recommendations on the choice of rational hydraulic regimes of oil pipelines, as well as to outline the ways for the directed synthesis of high-performance polymer additions that reduce friction in the turbulent oil pipelines.

In the hydrodynamics of polymer solutions there takes place the transition from accumulating experimental information to understanding the physical essence and establishing main regularities of manifestation of memory and elasticity effects. Toms effect revealed as an experimental fact in the late 40-ies, up to now has been causing great difficulties when interpreting it from the point of view of modern ideas of the hydrodynamics of turbulent flow.

Among the attempts to explain the nature of effect Toms', lying in drag reduction by the polymeric components, special place is taken by a hypothesis, based on strong deformation effect of a near-the- 
wall turbulence on macromolecules $[17,45]$. For the substantiation of this hypothesis experimental proofs of presence of large degrees of deformation of macromolecules in a wall-adjacent zone of a turbulent flow are necessary. The skepticism concerning strong deformation effect of wall-adjacent turbulence on macromolecules is stipulated yet by the fact that, as a rule, shift effects wall-adjacent a turbulence are analyzed, and not the, jet flows ("explosions") with a longitudinal gradient of speed which arise in the wall-adjacent area $[37,51]$. It is possible to hope, that the way to understanding and describing phenomena reduction of turbulent friction by polymer additions lies through the study of hydrodynamic effects of big reversible (as well as non-reversible) deformations of molecular coils in flows with stretching.

Therefore the experiments proving the stretching of molecules in conditions of wall-adjacent turbulence have a fundamental character not only in point of developing the mechanism of drag reduction by polymer additions but also in point of more profound insight into the nature of turbulence itself.

For polarization-optical visualization of wall-adjacent turbulence flow of polymer solutions using the experimental installation, consisting of the optical bench, the light source, a polarizing microscope, filter and a plate equal to a quarter of a wavelength. The scheme of the installation can be found in the $[2,30]$. The light source was a mercury-quartz lamp DRSh250. Transparent rectangular channel was used as hydrodynamic cells. It was made of glass and had the cross-section $(4 \times 4) \cdot 10^{-3} \mathrm{~m}$ and the length of $1 \mathrm{~m}$. It was fixed to the special coordination table and could be moved in three mutually perpendicular directions. Visualization was made in the wall-adjacent area of the channel, that was located at a distance of $0.25 \mathrm{~m}$ from its end.

Due to the significant experimental difficulties in obtaining data on the stress state of macromolecules in conditions of wall-adjacent turbulence in the oil flow, experiments were carried out with water solutions of polyethylene oxide (PEO), which were used as a model polymer solutions in oil.

PEO having the viscosity-average molecular weight of $4 \cdot 10^{6}$ was used as a polymeric additive and latent root of a The solutions of polymer had concentration $0.01-0.05 \%$. Special hydrodynamic canal a rectangular cross-section $(4 \times 4) \cdot 10^{-3} \mathrm{~m}$ and height of $1 \mathrm{~m}$ was used. 
Visualization made in wall-adjacent of area located on a distance $0.25 \mathrm{~m}$ from its extremity of the channel.

Deformation of the Molecular coils of Flexible Polymers under wall-adjacent turbulence conditions. Experiments proving macromolecule unrolling under wall-adjacent turbulence have fundamental character not only in relation to the development of the Toms' effect mechanism but as well as to the more profound insight of the nature of the wall-adjacent turbulence itself.

Such tests can be carried out due to polymer linear macromolecules having their own anisotropy and anisotropy of form. Moreover in case of full macromolecule unrolling that can be realized only in flows with stretching, there takes place rather significant (by $3 \div 4$ orders of magnitude greater than for simple shift) and quick increment of the birefringence factor with the increase of velocity gradient acting on molecular coils up to extremely possible $\Delta n_{\infty}$ [20]. The given peculiarity of birefringence dependence on the degree of macromolecule unrolling just gives hope for the occurrence of birefringence area in zones with greater longitudinal velocity gradients of a boundary layer despite the sharp sensitivity reduction of the polarization-optical method under small polymer solution concentrations.

Fig. 3.1 shows a sketch of a picture birefringence zone of a turbulent boundary layer of a current of a water solution PEO having concentration $0.05 \%$ and $\mathrm{Re}=2 \cdot 10^{4}$.

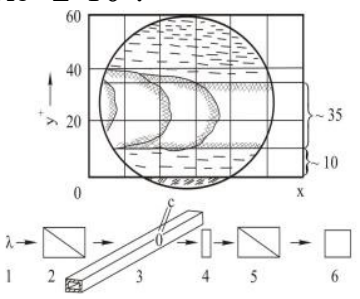

Fig. 3.1. The sketch of a picture of birefringence zone and a diagram of experimental installation 1 - mercury lamp; 2 - polarizer; 3 -hydrodynamic channel; 4 - plate a equal to a quarter of a wavelength; 5 - analyzer; 6 - camera or cine camera

Polarization and optical visualization testify, that in the field of $10 \leq y^{+} 45$, in which maximum generation of jets of fluid happens, birefringence is localized. In the immediate proximity to the wall $\left(0 \leq y^{+}<10\right)$ and in the field of the kernel $\left(y^{+}>45\right)$ birefringence is unvariable $\left(y^{+}=\mathrm{u}^{*} \cdot y / v\right.$, where: $y$ - is coordinate; $\mathrm{v}^{*}$ - is dynamic velocity; $v$ - is kinematic viscosity). 
This fundamental experiment unequivocally shows the presence of strong deformation effect on the molecular coils in certain zones of a turbulent boundary layer, having supercritical longitudinal gradients of speeds.

A mean angle of the orientation of polymer molecules with a direction of the main flow is $\sim 35^{\circ}$. The performed visualization of near-the-wall turbulent flow of water solution PEO has confirmed the conventional ideas about the structure of a turbulent boundary layer.

Adequate experimental verification of macromolecule unrolling under wall-adjacent turbulence made it obvious that using nonturbulence flows with stretching for the study of macromolecule interaction with hydrodynamic field is advantageous as giving the possibility to experimentally investigate "abnormal" effects in controlled conditions and allowing to model the main peculiarities of a turbulence boundary layer.

Dynamic structure formation in solutions of hudrodynamic active polymers. Let's examine the tests allowing to reveal the ability of hydrodynamic active polymers to dynamic structure formation effected by hydrodynamic field with stretching. To create hydrodynamic field with stretching there has been used a flow of Newtonian liquid (water, acetone, dioxan) converging to a small outlet $0.3 \cdot 10^{-3} \mathrm{~m}$ in diameter. At quite a distance from the outlet there have been injected in this flow some jets of PEO solutions or hydrolyzed polyacrylamide (HPAA) with molecular mass $6 \cdot 10^{6}$ and $4 \cdot 10^{6}$ and characteristic viscosity 2.42 and $3.70 \mathrm{~m}^{3} / \mathrm{kg}$ correspondingly. The degree of PAA hydrolysis was 5 percent. Interval of the studied concentrations was $0.001 \div 0.1 \%$. Polymer solution velocity in points of injection agreed with velocity of the main liquid flow. Visualization of the flow in the inlet area was done with the help of dye additives injected into polymer solution. Under small outflow velocities dyed jets of polymer solution visualize stream-lines of the main flow (fig. 3.2). 

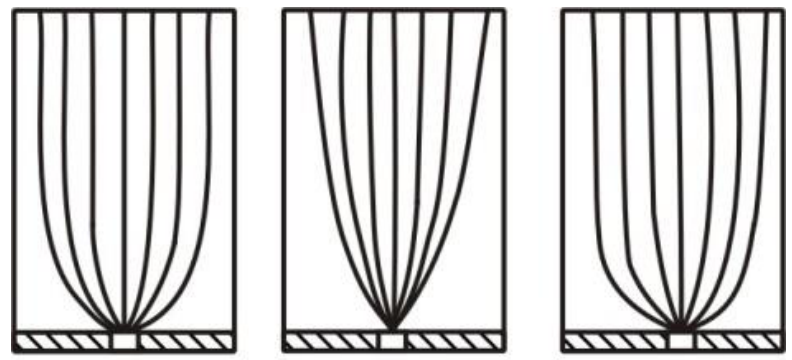

Fig. 3.2. Picture of flow in the inlet area of a capillary when injecting polymer solution into water during pre-critical mode of outflow $\mathrm{MPEO}=6 \cdot 10^{6} ; \mathrm{CPEO}=0,025 \%$; $\mathrm{M}_{\mathrm{HPAA}}=4 \cdot 10^{6}, \mathrm{C}_{\mathrm{HPAA}}=0,05 \%$. $a$ - water into water at $\dot{\varepsilon}>\dot{\varepsilon}_{c r} ; b$ - HPAA into water at $\dot{\varepsilon}>\dot{\varepsilon}_{c r} ; c$ - PEO into water at $\dot{\varepsilon}<\dot{\varepsilon}_{c r}$

In his case behaviour of PEO and HPAA solution jets doesn't differ at all from the behaviour of water jets injected into converging liquid flow. Reaching some critical flow rate of liquid through the orifice the character of jet flow of polymer solution drastically changes. Rather thick polymer jets transform into thin threads that change their length with time flow (fig. 3.3).

When observing the dynamics of forming and destructing separate threads (here lies the moving pulsation character of the flooded polymer jets) the following regularity comes to life. At the beginning when polymer jets approach the orifice there can be traced their gradual bend towards the orifice. Here their velocity growth along these curve trajectories becomes more noticeable as the jet thickness gets reduced. Near the orifice discontinuous (for PEO solutions) reduction of jets thickness takes place as they are transformed into thin threads. Transformation area of a thick jet into a thin thread starts to shift up along the jets resulting in the increase of the thread length. HPAA water solutions reveal more gradual change of jet thickness than PEO solutions. As jets approach the orifice not concurrently the length of threads spun by the flow from separate polymer jets is different at each given moment. Birefringence is observed in the area of thread emergence. 


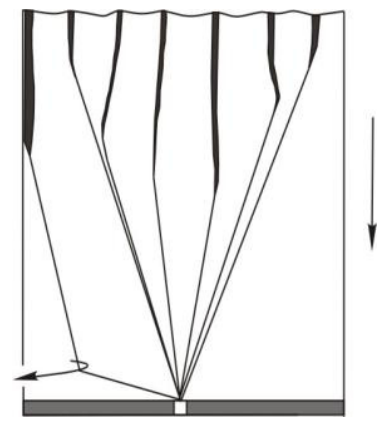

Fig. 3.3. Photo of flow at the point of wire probe effecting a polymer thread spun by hydrodynamic field $\mathrm{M}_{\mathrm{PEO}}=6 \cdot 10^{6}, \mathrm{C}_{\mathrm{PEO}}=0.03 \%, \bar{u}=2.5 \mathrm{~m} \cdot \mathrm{s}^{-1}$

When reaching some critical length a thread cuts off in close proximity to the orifice. After that the remaining at the top thread loses its elasticity and sags marking one of the stream lines of the main flow. When a polymer jet approaches the orifice again the whole thing is repeated anew. This process is resumed with time interval from fractions of a second to several seconds depending upon the outflow mode of the main flow, polymer molecular mass, polymer type, solvent quality and temperature, as well as polymer concentration in solution.

Outflow velocity growth of converging water flow as well as increase of molecular mass and polymer concentration result in the increase of thread length and reduction of their length-changing rate. Temperature growth leads to the opposite effect. Tests with acetone and dioxan (solvents with poorer thermodynamic qualities than water) showed that under the same velocities as in tests with water pulsation rate of thread length is bigger, but the amplitude of these changes is less than in case of injecting polymer solution into water. It should be also stressed that there are such ejection modes for concentrated (to Debay $[\eta]_{0} \cdot C>1$ ) polymer solutions, when the emerged polymer threads don't cut off during the whole period of observation.

There also has been studied the effect of initial (non-turbulent by hydrodynamic field) sizes of molecular coils, that in polyelectrolytes (HPAA) depend on the hydrogen measure of the surroundings, on the ejection character of HPAA solution jets injected into water flow. The experiment has shown that in case of $\mathrm{pH}$ of the 
main flow equaling 2 the length of a polymer thread decreases sharply (3-4 times for 0.05 percent concentration) while the thread lengthchanging rate increases by $1.5-2$ times. The increase of $\mathrm{pH}$ in the range between 7 and 9.5 practically doesn't influence the ejection character.

The main regularities of the polymer jets behaviour when injected into a flow with stretching, which is the main liquid flow, are similar to those in case of polymer solution outflow through short capillaries $[17,45]$. Hence, the observed behaviour peculiarities of the flooded polymer jets in flow with stretching are caused by rather strong polymer coils unrolling effected by hydrodynamic field (the degree of unrolling here reaches $\sim 60-70$ percent $[2,33]$ ). Uncoiling of macromolecule chains leads to decrease of their flexibility, which under certain conditions causes phase separation effected by hydrodynamic field, i.e. it leads to dynamic structure formation.

The following experiment may serve as a proof of dynamic structure formation effected by hydrodynamic field with stretching. A thin wire with a device for polymer thread hold-up is introduced into the inlet area of the orifice during the pre-critical mode of the main flow ejection. This wire travel in the plane perpendicular to jets doesn't change the flow picture, i.e. during these modes the polymer jets are permeable to it. If the experiment is repeated during over-critical mode of liquid outflow, then it would be possible to hold-up one or several polymer threads during their growth and divert them as it is shown in fig. 3.3. This can be done only if there is a strong interaction between polymer molecules, i.e. when sub-molecular structure is formed, in this case under the effect of stretching hydrodynamic field. The minimum concentration of PEO water solutions allowing to divert a thread spun by hydrodynamic field was 0.008 percent, which corresponds to the range of diluted solutions $\left([\eta]_{0} \cdot \mathrm{C}<1\right)$.

The given results as well as those obtained while studying converging flows of polymer solutions allow to state that hydrodynamic active polymer solutions should be divided into at least three concentration ranges. The first is the range of diluted polymer solutions when macromolecules are under strong deformation effect (unrolling) caused by hydrodynamic field, but the interaction between them is still weak. The second is the range of semi-diluted solutions (intermediate) when during pre-critical flow modes become diluted 
and during over-critical modes they become concentrated due to super-molecule structure formation caused by the shift of phase separation curves effected by hydrodynamic field. These dynamic structures should be classified as dynamic phase transitions that are studied by the theory of dissipation structures [42]. And the last range is the one of concentrated solutions $\left([\eta]_{0} \cdot C>1\right)$, when noticeable interaction between molecules do exist without hydrodynamic field. It should be stressed that the first two ranges refer to concentrations when the Toms' effect is manifested.

So, the results obtained by us show that dynamic structure formation and periodic processes subjected to Prigozhin's principles of self-organization may occur in hydrodynamic active polymer solutions in flow with stretching.

Polymer solutions in model conditions of wall-adjacent turbulence. When changing the orientation of zones with stretching generated with a help of a short capillary tube as to the wall (barrier) position it is possible to simulate ejections of polymer solution micro-jets from the wall-adjacent area.

Fig. 3.4 shows curves that characterize relationship of effective viscosity to $\mathrm{u}$ and obtained in PEO water solution flow through a short capillary tube with orientation of a single axis stretching zone along a normal to the "wall" surface. It is seen that when a stretching zone approaches a solid surface the flow becomes more dissipative, but the critical velocity is shifted to less values. The given situation may serve as a model of normal component in a blasting flow near the wall; then the data obtained testify to the decrease of probability for such "blasts" to occur in the wall-adjacent area when there is a polymer in a liquid. That's why the thickness of a viscous sub-layer in a liquid with decreasing resistance must be higher and the average (in time) intensity of lateral velocity pulsations must be less than in a liquid without a polymer. 


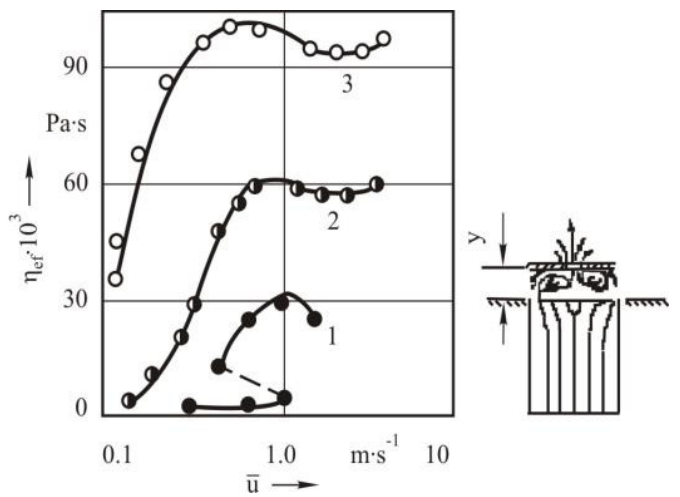

Fig. 3.4. Influence of a space the wall positioned normally to the axis of a longitudinal flow on effective viscosity in polymer solution $M_{P E O}=4 \cdot 10^{6} ; C_{P E O}=0,05 \% ; y$ : 1 - without a wall $(y \rightarrow \infty) ; 2-5 \cdot 10^{-3} \mathrm{~m} ; 3-5 \cdot 10^{-3} \mathrm{~m}$

Curves shown in fig. 3.5 meet the conditions of PEO solution outflow through a capillary tube when its axis is positioned in parallel to the "wall" surface. When surface approaches zone axis with longitudinal velocity gradient the maximum value of effective viscosity is practically unchanged while the critical velocity value is shifted to greater values.

This situation serves as a model of longitudinal component in a blasting flow near the wall. Independence of the maximum viscosity value and insufficient increase of $u_{\mathrm{cr}}$ on approaching the "wall" must testify to the fact that average (in time) intensity of longitudinal blasts and, thus, velocity pulsations near the wall are similar to those in flows without polymer additives.

In flows with stretching zones macromolecules of a dissolved polymer, when $\dot{\varepsilon}>\dot{\varepsilon}_{\mathrm{cr}}$, suppress big longitudinal gradients, and just this very feature must lead to the decrease of velocity pulsation intensity near the wall and manifestation of some threshold flow mode that gives way to the hydrodynamic activity of polymer additives to be revealed. The obtained results are in complete conformity with the known ones that prove the existence of a threshold velocity of Toms' effect manifestation [41]. 


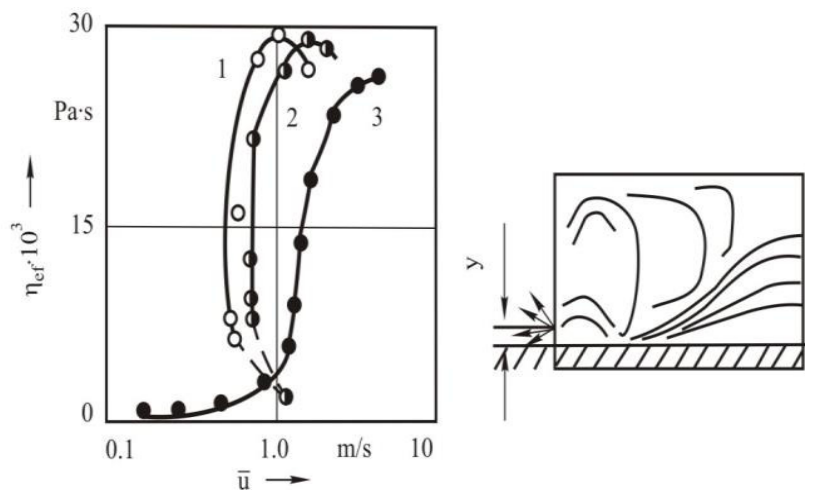

Fig. 3.5. Influence of a space to the wall positioned in parallel to the axis of a longitudinal flow on effective viscosity in polymer solution $\mathrm{M}_{\mathrm{PEO}}=4 \cdot 10^{6} ; \mathrm{C}_{\mathrm{PEO}}=0,05 \% y ; y: 1-$ without a wall $(y \rightarrow \infty)$ $2-5 \cdot 10^{-3} \mathrm{~m} ; 3-5 \cdot 10^{-4} \mathrm{~m}$

Summarizing we may say that the studied experiments and the data obtained allow to receive additional information on the structure of wall-adjacent turbulence and to come to the issue on mechanism of turbulence friction reduction effect due to polymer additives; we are to deal with it in the following section.

Mechanism of turbulence friction reduction effect due to polymer additives. The data obtained due to this work convincingly prove that the system water-polymer (polymer with flexible chains) under walladjacent turbulence in certain flow modes $\left(v^{*}>v^{*}\right.$ treshold $)$ is selfregulating with negative feedback that is manifested in the following: the growing longitudinal velocity gradients acting in the area $\left(0 \leq \mathrm{y}^{+}<10\right)$ where jet flows are generated to the maximum as a result of turbulent explosions, lead to macromolecule coil deformation which in its turn causes structure reconstruction of these flows in order to decrease longitudinal velocity gradients. The latter cannot be lower than critical values equaling $0,5 / \theta_{\mathrm{c}}$.

If they appear to be lower than $\dot{\varepsilon}_{c r}$ then macromolecules will start to form into a coil with no more effect on velocity field; velocity gradient sharply increases here leading to significant deformation of macromolecular coils and everything repeats again. It means that in auto-vibration mode the system regulates velocity gradient in jet flows making it relatively small but still sufficient enough for mac- 
romolecular coil deformation. It should be stressed here that namely this ensures Virk's asymptote and no full laminarization of walladjacent turbulence flow.

Growth of deformation velocity as a result of non-linear effects leads to insignificant further increase of deformation factor, but this turns to be enough to stabilize typical velocity gradient within a rather broad interval of flow velocities. In case of rather high $v^{*}$, as it comes out from $[43,51,41]$ deformation velocity growth leads to deformation factor decrease due to the cut of effect time for longitudinal velocity gradient. The latter is due to small-scale turbulence which share sharply increases under high $v^{*}[43,51,41]$.That's why Toms' effect dependence on velocity has extremal nature. Degradation of polymer solutions starts to contribute significantly to Toms' effect reduction at high velocities.

So, liquid ejections from the wall area into the outer area of a boundary layer that nearly periodically interrupt calm flowing, are stabilized by feedback molecule deformation (flooded jet flows with longitudinal velocity gradient). This leads to frequency decrease of these ejections and finally to suppression of normal Newtonian energy processes caused by jet ejections and, thus, to friction resistance decrease. The part of explosion cycle where gradient $\dot{\varepsilon}>\dot{\varepsilon}_{c r}=0,5 / \theta_{c}$ takes place is subjected to the greatest effect. The interaction between macromolecular coils and hydrodynamic field in auto-vibration mode takes place in any zone of a flow with stretching in a wall-adjacent area of a turbulence flow, which prevents primary turbulence development.

It is important to compare macromolecular scales describing polymer molecules in solution as well as turbulence scale near the wall. In this case linear and temporal scales must be single-valued. Analysis of experimental data shows that both typical size $\lambda_{f}$ and time $\mathrm{T}_{\mathrm{f}}$ depend on concentration, molecular mass and some other factors. On average the following factors can be accepted: $\lambda_{f} \sim 10^{-4}-10^{-5} \mathrm{~m}$, while $T_{f} \sim 10^{-3}-10^{-4} s$ [41]. Macromolecules in solution are characterized by a linear scale that depends on the degree of their stretching; contour length of a completely stretched macromolecule should 
be taken as its characteristic feature, maximum (fundamental) time for relaxation should be treated as typical time.

In case of full macromolecule stretching its contour length has a micro-scale factor of wall-adjacent turbulence. The stretching rate of approximate $60 \div 70$ percent was obtained in model conditions of wall-adjacent turbulence. This demonstrates the fact that interaction between turbulence field velocity and macromolecules may take place both on the level of individual molecules (very watered down solutions) and on the level of super-molecular formations generated by hydrodynamic field (semi-diluted and moderately concentrated solutions). The average relaxation time of polymer solutions $[1,41]$ coincides in factor with the typical time of wall-adjacent turbulence.

Comparison of concentration dependence of the Toms' effect and the data testifying to the formation of dynamic super-molecular structures in polymer solutions allows to state that in the vicinity of optimal concentration (when $C \geq C_{\text {opt }}$ ), where the Toms' effect reaches its maximum, solutions start generating anisotropic super-molecular forms having the lifetime 10-20 times longer than $\theta_{c}$ [2,45]. Further increase of concentration leads to conditions favourable for interaction between individual polymer molecules even without the hydrodynamic field effecting on them. The longitudinal hydrodynamic field effect in polymer solution gives way to the formation of dynamic super-molecular structures with lifetime significantly exceeding (in several factors) the temporal scale of the wall-adjacent turbulence. That's why unlike the super-molecular forms in semi-diluted solutions these super-molecular forms act as "stiff sticks". As an outcome of this the Toms' effect is decreased at a high polymer concentration in solution as well as at a high dynamic velocity.

On the basis of data that characterize macromolecule dynamics in non-turbulence flows with stretching and the proved evidence of strong deformation effect on macromolecules in wall-adjacent turbulence, and using data of model studies of turbulence peculiarities in a boundary layer there has been established the molecular-andsupermolecular mechanism of effect reduction for flow resistance when injecting soluble polymer additives in a turbulence flow. Mechanism of Toms' effect lies in the occurrence of auto-fluctuating mode of reversible processes of macromolecule deformation caused 
by longitudinal velocity gradients that quasi-regularly originate in turbulence boundary layer and in macromolecule deformation effect both on molecular (when $C<C_{\text {opt }}$ ) and super-molecular (when $C>C_{\text {opt }}$ ) levels on the wall-adjacent turbulence structure, i.e. as a result of macromolecule deformation oscillations and solubility of dynamic super-molecular forms brought about by the flow-with-stretching effect. All this leads to the increase of liquid ejection periods into the outer zone of the boundary layer and in consequence to the viscous sub-layer becoming thicker. As an outcome of this generation of primary turbulence gets reduced and general level of turbulence dissipation in the flow becomes lower. In case of sufficiently big molecular masses and concentrations the viscosity growth caused by both "common" intermolecular interaction and dynamic structure formation leads to sharp Toms' effect decrease.

The considered experimental data prove the substantiation to transfer outcomes obtained during the study of macromolecule dynamics in non-turbulence flows with stretching onto jet currents of wall-adjacent ejections in turbulence flow, i.e. turbulence current (to macroscopic scale) is perceived as laminar one (to microscopic scale) when hydrodynamic field interacts with polymer molecules. Macromolecules may serve as an efficient tool of getting additional information about the structure of wall-adjacent turbulence.

The developed approach of explaining the turbulent drag reduction mechanism fits well into the general scheme of self-regulatory processes, which are dominated by negative feedbacks. It is typical for systems that can change their properties under the action of external physical effects, in this case, under the influence of jet currents ("explosions") with stretching which locally occurring in the boundary layer of the oil pipeline.

Understanding the nature of reducing drag flow of oil in pipelines by small polymer additions will allow to develop recommendations on the choice of rational hydraulic regimes of oil pipelines, as well as to outline the ways for the directed synthesis of high-performance polymer additions that reduce friction in the turbulent oil pipelines.

\section{Improvement of Water Fire-Fighting Systems at Oilfield Territories}

A fire (fires) is a major ecological factor that at certain conditions leads to the strongest contamination of environment. An increase of 
work efficiency of the fire extinguishing systems is one of important engineer-technological measures that allows to decrease the influence

of the combustion products on a biosphere and to increase level of safety on oilfield territories. Reduction of hydrodynamic resistance of fire hoses and pipelines allows to improve the efficiency of waterbased fire-suppression systems which are the most widespread and feasible methods of the fire-fighting on the oilfield territories.

Among the well-known methods of the decrease in the hydrodynamic drag resistance through the artificial modification of the boundary layer of the fire hoses, the method of the introduction of the polymer solutions is almost unique, and certain practical results have been achieved in its development. The study has shown that the introduction of small amounts of polyethylene oxide (PEO) and polyacrylamide (PAA) into the fire-suppression liquids (water and water solutions of surfactants) make it possible to significantly (down to $75 \%$ ) reduce the hydrodynamic drag resistance. This reduction (when other parameters of the hydraulic system are unchanged) ensures that the capacity of the fire-fighting systems is 1.5-2 times higher, or the length of the fire hoses may be 3-5 times higher, or the pump power consumption may be decreased by $60-70 \%$, or the diameter of the fire-suppression pipes may be decreased by $15-20 \%$. Besides, it has been shown that the additions of the polymers to water or surfactant solutions considerably improve their firesuppression properties [2, 52-54].

This study relates to the hydrodynamics of the polymer solutions in the pipelines and problem of the improvement of the introduction devices. The hydrodynamics of polymer solutions at the polymer introduction into the boundary layer of the fire hoses has not been examined properly.

It is assumed that in case of the flow of polymer solutions through the slots and other elements of the introduction systems, essential "anomalies", which could considerablyaffect the Toms effect, cannot be observed. Such a conclusion is based on the analysis of the data obtained from the study of the shear laminar flows where the effects of elastic deformations are insignificant. In the introduction systems a complex flow is dominant and it consists of superposition of the shear and predominantly longitudinal (with stretching) flow. In case 
of such complex flows, the effects of elastic deformations become so significant that it may result in lessening the potential effect of the polymer additives, especially at high velocities of the flow of the firesuppressing liquids.

Water solutions of polyethylene oxide with the molecular masses $2 \cdot 10^{6}$ and $410^{6}$ were investigated. The mass fraction of polymer was varied from 0 to $0.3 \%$, temperature during experiments: $25^{\circ} \mathrm{C}$.

Solutions of fully dissolved polymer were prepared in dark vessel by dissolving the ethanol-polymer suspension in distilled water at room temperature during 2-3 days or by dilution of the previously prepared solutions of polymer $(0.1 \%, 0.2 \%$ and $0.5 \%)$ during $7-8$ days before the desired concentration. Since water solutions of PEO significantlychange properties at the upon prolonged storage, conformities to law of their aging process in water were preliminary studied and stabilizers to exclude it were chosen. The addition of $0.05 \%$ by mass potassium iodide solution in the PEO and PAA, which almost completely eliminates the aging of these polymers in water during storage and does not affect on the hydrodynamic activity, are used as such stabilizer [1,2].

We used a special hydrodynamic bench that allows achieving the exhaust velocities of the water flow through its channel of up to $35 \mathrm{~m} / \mathrm{s}$; the channel's length was $8.5 \mathrm{~m}[1,55]$. Orifices for measuring the pressure and for the sensors of friction force were placed on the lower wall of the channel. The injection system consisted of a dosing unit, underslot chamber ensuring different conditions of the deformation of the polymer solution (by changing the entrance angle) at the entrance to the slot. The angle between the injected polymer stream and the wall did not vary. The following characteristics have been variable: the angle of the opening of the slot $\beta^{\circ}$ (the angle of the entrance to the slot), concentration of the injected polymer solution, velocity of the injection, molecular mass, polymer brand, and velocity of the fire suppressing liquid (water).

This paper regularities and manifestations of the elastic deformations of the polymer flow are investigated under conditions close to that of the real flows of the fire hoses at oilfield territories.

The experiments described below were conducted in order to establish the conformities to law of manifestation of elastic deformations in the flow of polymer solutions under conditions 
typical for the internal problem in relation to the fire hoses and pipelines.

In fig. 4.1, the experimental data related to the flow of polyethylene oxide (PEO) water solutions through the underslot camera are shown. It is clear that the phenomena, unusual for purely viscous mediums, are inherent for such flows. At a certain critical (threshold) average exhaust velocity $\bar{u}$, the relative pressure differential increases sharply, and it is sharper at the higher polymer concentrations. The characteristics of the dependence $\xi=f(\bar{u})$ indicate the high dissipation of energy in the polymer solutions flow through the injector, i.e. the increased hydrodynamic drag resistance is observed at the supercritical flow rates.

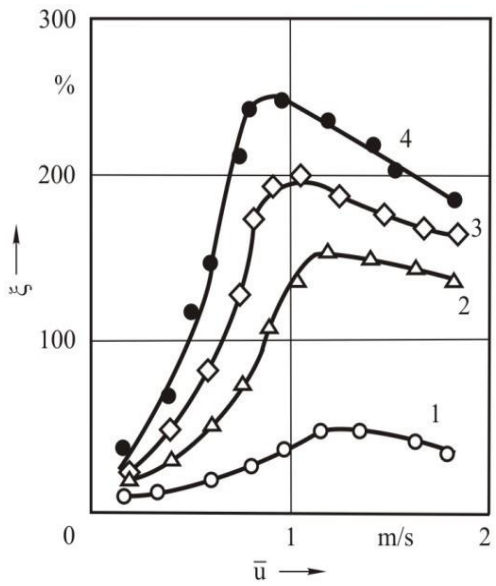

Fig. 4.1. Influence of $\bar{u}$ and angle of entrance into a slot on the relative pressure differential

Mol. mass: $4 \cdot 10^{6}, C_{\mathrm{PEO}}=0.1 \% ; \beta: 1-9^{\circ}, 2-13^{\circ}, 3-22^{\circ}, 4-34^{\circ}$

The presented experimental data are in accordance with the results obtained from the study of the flow of the polymer solutions under the simulated conditions such as through short capillary tubes and slots. These flows were thoroughly investigated in papers $[10,11,17,33,36,45]$. There should be stressed the most important moments of the elastic stress effects in the polymer solution flows with stretching. Transition to a flow mode with increased energy dissipation is accompanied by formation of so called "inlet flooded jet" as a "cord" or "fillet" enclosed by secondary flows in the shape of 
ring-shaped vortex. In case of the supercritical flow mode for polymer concentrations ranged from very diluted to moderately concentrated, the hydrodynamic field causes rather strong deformation effects on molecular chains. The uncoiled part of a polymer chain may be as large as $60-70 \%$ [33]. In half-diluted and moderately concentrated polymer solutions, the relaxation times of the fully stretched and slightly deformed individual chains differ more than by 2 orders of magnitude.

The reason for such a large time for the curling of the polymer chain is supermolecular structures formed in the hydrodynamic field. This is reflected in the decrease of turbulent friction if the lifetime of supermolecular formations in the polymer solution at the moment of its introduction to the boundary layer is comparable to the residence time in the fire hose at oil field territories.

The results of the polymer solution injection onto the lower wall of the channel through the underslot chambers with varying angles of the entrance to the slot shows (fig. 4.2), that when the polymer solution is introduced onto the inner surface of the fire hose at low angles of the entrance to the slot, the drop in the tangent stresses of friction is exhibited practically right behind the point of the introduction of the polymer to the flow.

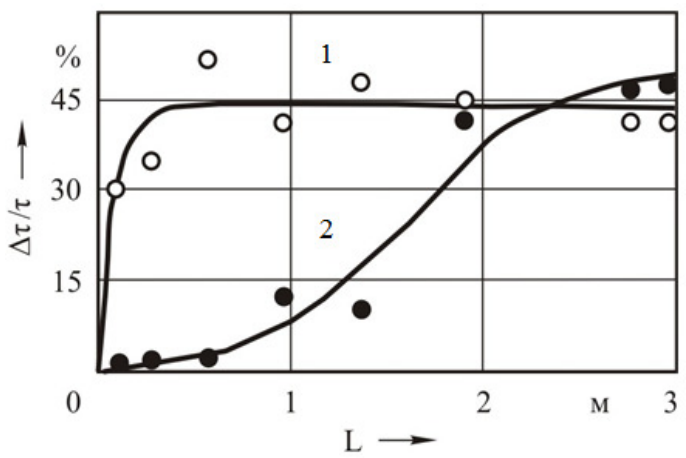

Fig. 4.2. Influence of the angle of the entrance to the slot on the distribution of the decrease in the tangent stresses along the lower wall of the channel for the injections of PEO solutions

Mol. mass: $2 \cdot 10^{6}, C_{\mathrm{PEO}}=0.3 \%, V_{0}=16.5 \mathrm{~m} / \mathrm{s}, Q=50 \mathrm{sm}^{3} / \mathrm{s} ; \beta^{\circ}: 1-7.8^{\circ}, 2-165^{\circ}$

If the polymer solution is introduced into the boundary layer through the chamber with a large angle of the entrance, there is a de- 
lay in development of hydrodynamic activity of the polymer molecules.

It should be mentioned that the distribution of the tangent stresses and relative pressure losses along the channel correlate with each other. As it follows from the results shown in fig. 4.2, the change of the mode of the polymer introduction through the underslot camera from weakly dissipative to strongly dissipative by modification of the entrance conditions results in double decrease in the drag resistance coefficient.

It should be mentioned that the distribution of the tangent stresses and relative pressure losses along the channel correlate with each other. As it follows from the results shown in fig. 4.2, the change of the mode of the polymer introduction through the underslot camera from weakly dissipative to strongly dissipative by modification of the entrance conditions results in double decrease in the drag resistance coefficient.

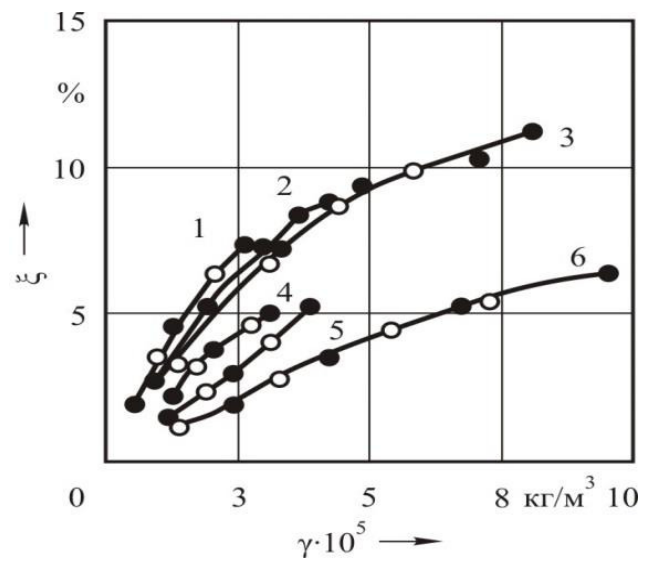

Fig. 4.3. Plot of the general pressure losses along the channel versus the specific concentration of PEO Mol. mass: $2 \cdot 10^{6}, \mathrm{~V}_{\mathrm{o}}:-16.5 \mathrm{~m} / \mathrm{s}, \mathbf{O}-25 \mathrm{~m} / \mathrm{s}$;

$\beta=7.8^{\circ}(1,2,3), \beta=165^{\circ}(4,5,6) ; C_{\mathrm{PEO}}: 1$ and $4-0.05 \%, 2$ and $5-0.1 \%, 3$ and 6 $-0.3 \%$

There has been (fig. 4.3) registered a considerably larger divergence in the plots of the drop of the resistance versus the changing concentrations of the polymer in case of the introduction of the polymer onto the inner surface of the fire hose under conditions of 
strong deformation effect of the hydrodynamic field on the injected solution, compared to the weak gradient effect.

Specific concentration was used to plot a chart (fig. 3)

$$
\gamma=Q \cdot C_{P E O} / \Omega \cdot V_{0},
$$

where $Q$ - volume flow rate of the injected liquid, $C_{P E O}$ - concentration of the injected polymer solution $\left(\mathrm{kg} / \mathrm{m}^{3}\right), \Omega$ - moistened surface, $V_{0}$ - velocity of the filling flow.

The visualization of the flows of the polymer solution in the underslot chamber shows that the conditions of the entrance render influence on the drop of hydrodynamic resistance only when there is a loss of stability of the flow caused, as was shown earlier in $[34,55]$, by the formation of the dynamic supermolecular structures which sharply increase the dissipativeness of the flow. The reduction of efficiency of the polymeric solution due to the deformation effects in the introduction system may be as large as $25 \%$ or higher at $\mathrm{V}_{0}>15$ $\mathrm{m} / \mathrm{s}$. The increase in the rate of the water flow results in the expansion of the area with the reduced hydrodynamic activity of the polymer.

The significance of the area with the reduced hydrodynamic activity of the polymer introduced into the boundary layer is the more, the less is the length of the fire hose. It may be explained by the fact that the lifetime of the derivative structures under conditions of the flow with stretching is in the order of magnitude of $0.1 \div 0.2 \mathrm{~s}$ or higher $[2,34,45,55]$. This is the time during which the polymer after leaving the slot in the fire hose has reduced activity due to its memory. Obviously, the higher is the velocity of the water flow, the larger is the area behind the slot filled with the polymer solution under this condition, and its size is defined as

$$
l_{\lambda}=\theta_{\mathrm{sw}} \cdot V_{\mathrm{o}}
$$

where $\theta_{\mathrm{sw}}$ - time of structural relaxation of the supermolecular formations, $V_{0}$ - velocity of the filling flow.

Hence, for the velocity of the water flow of $25 \mathrm{~m} / \mathrm{s}$, this area should extend downwards along the stream to $2.5 \mathrm{~m}$, if $\theta_{\mathrm{sw}}=0.1 \mathrm{~s}$. The estimated size of the area with the reduced hydrodynamic activity of the polymer is in good correlation with the experimentally obtained results. 
The results of the experiments with the introduction of the polymer solutions of the various concentration through the underslot camera with the changing angle of the entrance (fig. 4.3) show that for the given specific average concentration of the polymer in the boundary layer the efficiency of the reduction of drag resistance is decreased with the growth of the concentration of the injected polymer solution, and it is the stronger, if the angle of the entrance is higher. In [56], it was outlined a hypothesis that viscoelastic effects (swelling of the jet) near the slot strengthen the pull of the polymer solution by the external boundary layer and result in a faster decrease in the concentration of the polymer on the inner surface of the pipeline. The results obtained in $[57,58]$ make us reconsider this hypothesis because the visualization of the flow behind the slot [58] and the actual concentration of the polymer in the boundary layer have not evidenced to the increased diffusion of the polymer. Most feasible is the explanation based on the impact of the effective viscosity (taken in its broad sense) that does not contradict to the results of the studies on the hydrodynamic activity of the polymers under conditions of the introduction of the polymer solution to the fire suppression pipeline. The dynamic structures of the polymer formed in the hydrodynamic field cause its compression [34], and this, of course, should cause a decrease in the diffusion of the polymer in the boundary layer.

The detected regularities of the manifestation of elastic deformations at the introduction of the polymer solution to the firesuppression pipeline allow suggesting a method for evaluation of the flow resistance of the fire suppressant liquid with the polymer additives in it. The flow resistance of the liquid in the pipeline at the introduction of the polymer solution to the boundary layer caused by the elastic deformations can be determined as

$$
X=\int_{0}^{\ell_{\lambda}} \chi(x) \tau_{w 0} d x+\int_{\ell_{\lambda}}^{\ell_{p}-\ell_{\lambda}} \chi(x) \tau_{w c} d x,
$$

where $\chi$ is the perimeter of the pipeline cross-section, $\tau_{w l}$ and $\tau_{w o}$ tangential stresses with the introduction of the polymer to the boundary layer and without it, $L$ - length of the pipeline, $1_{\lambda}$ - length with the reduced hydrodynamic activity of the polymer.

The data obtained in this study shows that in solving the problems of drag reducing of the fire suppressing liquid in the fire hoses and pipeline by injecting the polymer solutions in the boundary layer, for 
the development of the optimum system of the introduction, it is necessary to take into account possible effects of the elastic deformations. The decrease in the effect of drag reduction at the introduction of the polymer solution into the boundary layer of the fire hose is due to the combination of the deformational effects of the longitudinal hydrodynamic field developed in the system of the injection and molecular-concentration characteristics of the polymeric solution.

Thus it is possible to improve the effective systems of the polymer introduction into the fire hoses and therefore operation of the fire suppression systems at oilfield territories using water.

This paper demonstrates the ways to improve the efficiency of the systems of input of polymers in fire hoses and pipelines, and to improve the efficiency of fire-fighting systems by water and solutions of surfactants that will help to reduce pollution by combustion products and, obviously, improve the safety of working conditions on oilfield territories.

\section{Degradation of polymer solutions in a hydrodynamic field with a longitudinal velocity gradient}

Despite the great number of investigations on studying the infl uence of various factors on the degradation of polymers in solutions, their results are ambiguous, and a number of questions are still not clearly understood [59-63]. Changes occurring in polymer solutions under the action of a hydrodynamic field have not been satisfactorily explained yet; there are no substantiated ideas of the mechanism of the phenomenon. Investigations considering the reasons why the effi ciency is lost by polymer solutions under stretching-flow conditions are very few in number. Elucidation of the causes of polymer degradation under hydrodynamic action is necessary for developing methods to control its negative impact on the effi ciency of practical use of polymers.

Flow with a longitudinal velocity gradient can be implemented when a polymer solution is flowing through capillaries with different inlet angles. In the inlet part of the capillary, flow with a predominantly longitudinal velocity gradient is implemented [11], whereas in the channel of a smooth capillary with a diameter constant along the length, flow with a transverse gradient. Therefore, in capillary liquid flow, there are both a predominantly longitudinal flow (at the capil- 
lary inlet) and a purely shear flow (in the capillary). The shorter the capillary, the larger the share of the longitudinal flow in the general hydrodynamic pattern, and the longer the capillary, the larger the share of the shear flow. The share of flow with a transverse velocity gradient can also be increased by decreasing the angle of entry into the capillary. It is important to elucidate what kind of flow is responsible for the polymer degradation in water. Also, it should be noted that many kinds of flow, e.g., turbulent or fi ltration-type, can be reduced to a superposition of two kinds of flow: shear flow and stretching one [10].

In the experiments, use was made of a flow-type viscometer with replaceable hydraulic resistors (capillaries with different inlet angles, lengths, and diameters) whose geometry enabled us to assign different velocity fi elds and residence times of macromolecules in these fields. The degree of action of hydrodynamic fields on the solutions of polyethylene oxide (PEO), polyacrylamide (PAM), and hydrolyzed polyacrylamide (HPAM) was assessed by the loss of their effi ciency in reducing turbulent friction. The relative change in the coeffi cient of hydrodynamic resistance refl ects the efficiency (hydrodynamic activity) of a polymer in reducing turbulent friction. This efficiency was calculated by using an expression of the following form [64]

$$
E=\frac{\lambda_{s l v}-\lambda_{s o l}}{\lambda_{s l v}} \equiv \frac{\Delta \lambda}{\lambda}=\left[1-\left(\frac{t_{s o l}}{t_{s l v}}\right)^{2} \frac{\Delta P_{s o l}}{\Delta P_{s l v}}\right] \cdot 100 \%
$$

In this formula, the subscript "slv" refers to the solvent and the subscript "sol," to the solution. In computing, we determined the value of the effect of reduction in the hydrodynamic resistance of a tube of length $1.02 \mathrm{~m}$ and diameter $2.17 \cdot 10^{-3} \mathrm{~m}$ in the case of flow of aqueous polymer solutions with a concentration lower than the optimum one (when $C<C_{\text {opt }}$ ), which had been prepared from the solution exposed to a hydrodynamic field. The quantity $C_{\text {opt }}$ corresponds to the maximum on the concentration dependence $E_{0}=f(C)$. The true value of the pressure difference at the channel ends was determined with account taken of the corrections for the head loss due to the removal of the kinetic energy by the outfl owing jet [65]. Other kinds of loss turned out to be minor. It was found that their value was smaller than $0.5 \%$ of the pressure indicated by the manometer at the largest flow 
rate. Therefore, they were disregarded. The error in measuring the coeffi cient of hydrodynamic resistance $\lambda$ was $1 \%$. The degradation was evaluated as

$$
D=\frac{E_{o}-E}{E_{o}} \cdot 100 \%,
$$

where $E_{0}$ and $E$ were determined at a constant Reynolds number. In some experiments, the degradation was evaluated as the ratio $E / E_{0}$ at a constant pressure difference. In most cases for PEO solutions treated by the hydrodynamic field at higheraverage temperatures or with additions of salt, the quantity $D$ was determined at $25^{\circ} \mathrm{C}$, with $C_{\text {PEO }}$ being equal to $0.0002 \%$. The additions of salt from the PEO solution were removed, on exposure to the hydrodynamic field, using a dialysis bag. This was done for the degree of action of the hydrodynamic field on the polymer system to be monitored by the solutions (control and treated), which were under identical conditions at the instant of measurement. Conversely, in other works in which the infl uence of temperature and salts on the degree of degradation of polymer solutions was studied in different hydrodynamic fields, the latter condition was violated [59-63]. Neglect of this circumstance often generates lots of mistakes and errors when experimental results on degradation of polymer solutions are interpreted.

Fig. 5.1 gives experimental results on treatment of a $0.004 \%$ PEO aqueous solution. The rate of flow through capillaries had a value of $2.6 \mathrm{~m} / \mathrm{s}$. It can be seen that a tenfold flow of the solution through a capillary of diameter $0.34 \cdot 10^{-3} \mathrm{~m}$ having a straight portion of length $0.45 \cdot 10^{-3} \mathrm{~m}$ and a convergent portion of $10^{-2} \mathrm{~m}$ with an opening angle of $20^{\circ}$ (curve 2) leaves the effi ciency of reduction in the hydrodynamic resistance unaltered, in practice, compared to the untreated solution (curve 1 ). Curves 3 and 4 correspond to the treatment of the tested solutions on capillaries of the same diameter with an inlet angle of $180^{\circ}$ and a length of $0.45 \cdot 10^{-3} \mathrm{~m}$ and $45 \cdot 10^{-3} \mathrm{~m}$, espectively. The hydrodynamic activity of the treated solutions has decreased by the same value despite the fact that the capillaries differed by two orders of magnitude in length and hence in the residence time of macromolecules in the shear flow. Since the conditions of entry into the capillaries were identical, the residence time of macromolecules in the longitudinal hydrodynamic field was identical, 
too. Increase in the number of treatment cycles to 85 (curves 5 and 6 ) caused the solution's hydrodynamic activity to degrade, but the capillary length, as previously, did not affect the value of degradation. A comparison of curves 2 and 3 shows that the increase in the degree of longitudinal action on the system leads to a greater degradation. Increase in the residence time of macromolecules in the region with a longitudinal velocity gradient, which was achieved by increasing the number of convergent-divergent cells of the hydraulic resistor [10], also caused the degradation to increase (fig. 5.1, curve 7).

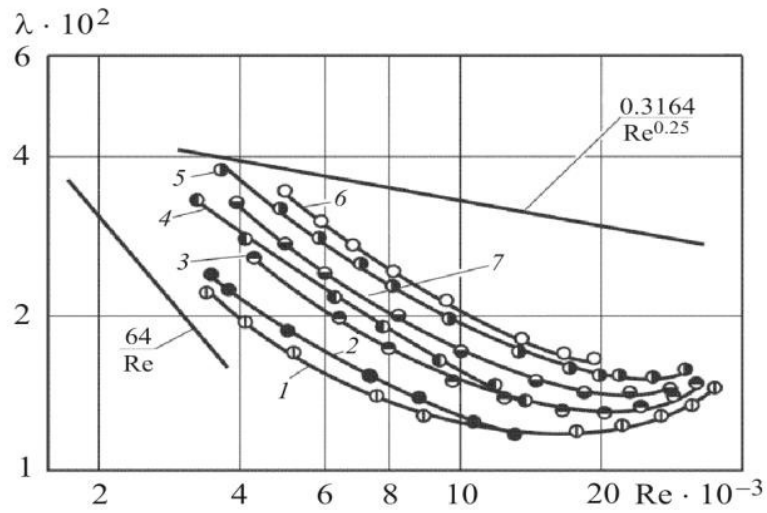

Fig. 5.1. Change in the hydrodynamic activity of PEO of mol. weight $6 \cdot 10^{6}$ on exposure to the hydrodynamic field: mic field with a predominantly transverse velocity gradient; 3 - in the hydrodynamic field with a redominantly longitudinal velocity gradient; 4 - in the superposition of shear and stretching fields; 5 and 6 - the same as 3 and 4, but with a longer time of exposure to the hydrodynamic field; 7 - in the hydrodynamic field of the hydraulic resistor with convergent-divergent cells

An analysis of the considered data shows that the degradation of polymer solutions is independent of the time of their residence in the laminar flow with a transverse velocity gradient and is fully determined by the value of the longitudinal velocity gradient and by the time of its action on the solution. Consequently, the degradation of polymer solutions in the hydrodynamic field is caused by the action of just stretching flows on macromolecules, whereas the shear component of these flows does not appreciably affect the degradation of the solutions. 

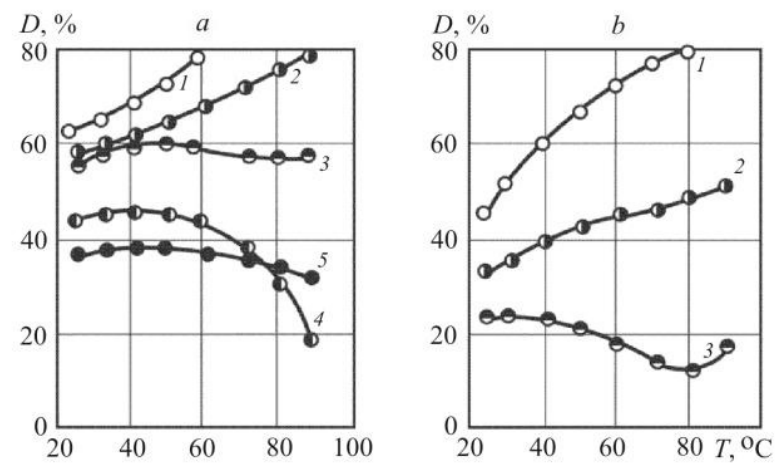

Fig. 5.2. Influence of temperature on the degradation of $P E O$ solutions on exposure to a stretching hydrodynamic field: $a$ - treatment included a 85 fold flow of a polymer solution at different temperatures through a capillary with an inlet angle at $180^{\circ}$ with a flow velocity of $2.6 \mathrm{~m} / \mathrm{s}$ at $M_{\mathrm{PEO}}=6 \cdot 10^{6}$ :

$$
\begin{gathered}
1-C_{\mathrm{PEO}}=0.0016 ; 2-0.004 ; 3-0.01 \text { and } 4-0.1 \% ; \\
M_{\mathrm{PEO}}=4 \cdot 10^{6}: 5-C_{\mathrm{PEO}}=0.1 \% ; b-M_{\mathrm{PEO}}=6 \cdot 10^{6} ; \\
\left.C_{\mathrm{PEO}}=0.0016 \%: 1\right) u=2,3-1.57 \text { and } 3-1.5 \mathrm{~m} / \mathrm{s}
\end{gathered}
$$

Increase in the molecular weight of the polymer and in the strength of the longitudinal hydrodynamic fi eld and decrease in the concentration of the polymer in the solution (increase in the concentration tends to suppress longitudinal velocity gradients) must cause the degradation to enhance. These conclusions are confi rmed by the data in fig. 5.2.

However, if the initial dimensions of macromolecules change due to the change in the temperature, the ionic force, and $\mathrm{pH}$ of the medium (figs. 5.2 and 5.3), the obtained results are not so unambiguous to interpret. It can be seen that the degradation of moderately concentrated solutions decreases with growth in the temperature, whereas that of diluted solutions increases.

Degradation decreases as the concentration of the salt in the solution grows, but if we increase the intensity of the hydrodynamic action (curve 3, fig. 5.3), degradation, at least in diluted solutions, increases. 


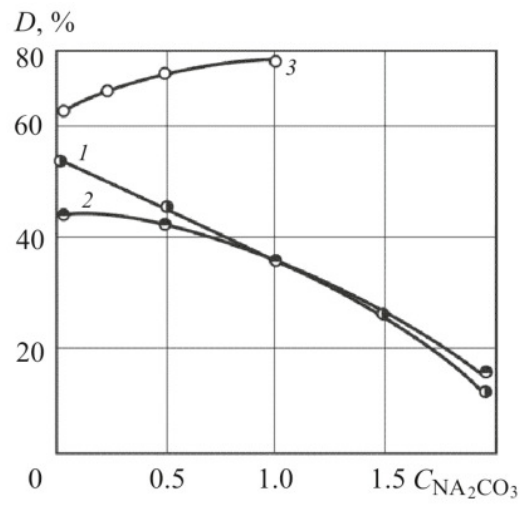

Fig. 5.3. Infl ence of the ionic force of the solution on the degradation of PEO of mol. weight $6 \cdot 10^{6}$ during the flow in a capillary with an inlet angle of $180^{\circ} ; u=2.6$ $\mathrm{m} / \mathrm{s}: 1-C_{\mathrm{PEO}}=0.004$ and $2-0.1 \% ; 3-u=8 \mathrm{~m} / \mathrm{s}$ and $C_{\mathrm{PEO}}=0.004 \%$

A comparison of the considered results and the data presented in [10] shows that if the polymer solutions are treated at velocities higher than $u_{\mathrm{cr}}$, increase in the temperature and the concentration of the salt causes the quantity $D$ to grow. The observed reduction in $D$ (figs. 5.2 and 3) is due to the treatment regime approaching a subcritical one. Analogous results have also been obtained on exposure of the polymer solutions to a hydrodynamic field implemented in the case of flow through porous media. It can be seen that the degradation of the aqueous PEO solutions grows with temperature (fig. 5.4).

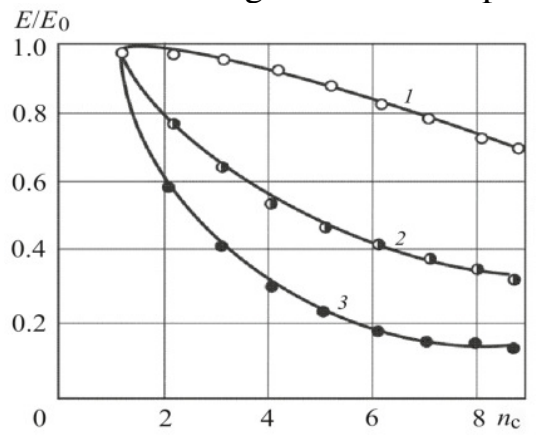

Fig. 5.4. Infl uence of temperature on the degradation PEO solutions of mol. Weight $3 \cdot 10^{6}$ during the flow through a porous tablet with a pore size of $1.6 \cdot 10^{-4} \mathrm{~m}$ at $C_{\mathrm{PEO}}=0.0002 \%$ and $\Delta P=3 \cdot 10^{5} \mathrm{~Pa}: 1-T=4 ; 2-40$ and $3-70^{\circ} \mathrm{C}$ 
In the PAM solutions, the influence of temperature is less pronounced (fig. 5.5, curves 1 and 2). Similar values of the characteristic viscosity of the PEO and PAM samples under study (1.4 and 1.1 $\mathrm{m}^{3} / \mathrm{kg}$ ) point to the nearly identical dimensions of unperturbed coiled macromolecules. Nonetheless, PAM exhibits higher stability to exposure to the hydrodynamic field than PEO. This could be due to the fact that the characteristic PEO viscosity, because of the presence of the lower critical mixing temperature in these solutions [18], and hence the dimensions of the volume occupied by the polymer coil, decreases as the temperature grows, whereas the characteristic PAM viscosity changes only slightly as the temperature grows.

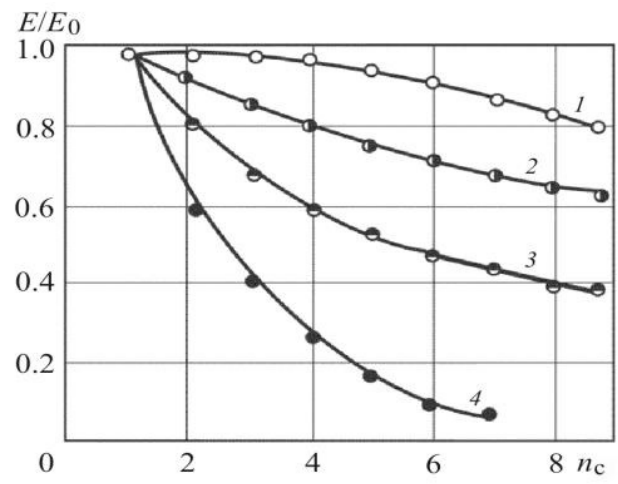

Fig. 5.5. Infl uence of temperature and $\mathrm{pH}$ of the medium on the degradation of PAM and HPAM solutions in filtration flow at $\Delta P=3 \cdot 10^{5} \mathrm{~Pa}$ and an average pore size of $1.6 \cdot 10^{-4} \mathrm{~m}$ :

$M_{\text {HPAM }}=6 \cdot 10^{6}$ and $C_{\text {PAM }}=0.0004 \%: 1-T=4$ and

$2-80^{\circ} \mathrm{C} ; M_{\mathrm{HPAM}}=6 \cdot 10^{6}$, the degree of hydrolysis is $12 \%$, $C_{\text {HPAM }}=0.0004 \%, \mathrm{pH}: 3-7$ and 4$) 3$

The conformation of HPAM molecules can be changed by varying the medium's $\mathrm{pH}$ from the dense reduced coil in the acidic medium to a loose, strongly swollen coil in the region of neutral and mildly alkaline $\mathrm{pH}$ values. The infl uence of the changes in the dimensions of the HPAM coiled macromolecules on the degradation of their solutions in filtration flow is presented in fig. 5.5 (curves 3 and 4). It can be seen from the fi gure that the highest stability of the HPAM solution is manifested in the neutral medium, despite the maximum dimensions of the polymer coils. 
The above-given results demonstrate that at a constant molecular weight, the reduction in the dimensions of polymer coils tends to enhance their degradation under the action of the hydrodynamic field with a longitudinal velocity gradient. This puts in doubt the decisive role of the reduction in the polymer's molecular weight due to the rupture of molecular chains, which has been used as the basis for the destruction mechanism of degradation [59-62].

The observed decrease in the characteristic viscosity of the polymer does not yet mean the reduction in the molecular weight and only points to the reduction in the hydrodynamic volume [66], i.e., in the equilibrium dimension of coiled macromolecules. Therefore, to confi rm the expressed assumptions, it is necessary to measure the molecular weight using absolute methods, among which are light scattering and sedimentation-diffusion analysis.

We investigated PEO of molecular weight $3 \cdot 10^{6}$ whose solution was exposed 150 times to a longitudinal hydrodynamic fi eld implemented in the capillary's inlet region in the supercritical regime of $\mathrm{fl}$ ow. Experimental data were processed by the Zimm method $[66,67]$.

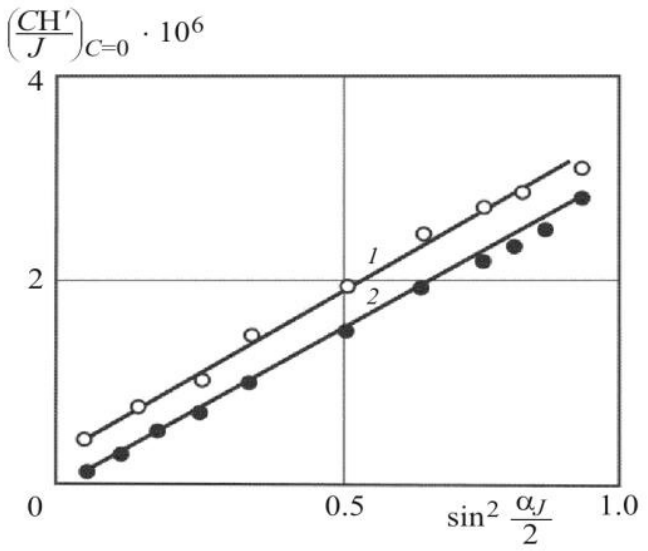

Fig. 5.6. Light-scattering indicatrix for the original and hydrodynamic-field-treated PEO solutions of mol. weight $3 \cdot 10^{6}$ at an initial concentration of the solutions of $0.1 \%$ : 1 - original polymer solution; 2 - after a 150 fold flow of the polymer solution through a capillary with an inlet angle of 180 with a flow velocity of $2.6 \mathrm{~m} / \mathrm{s}$ 
Fig. 5.6 gives the light-scattering indicatrices for the original (not treated by the hydrodynamic field) and hydrodynamic-field-treated PEO solutions. The results demonstrate the increase in the intensity of light scattering in the treated solution compared to the original one at all angles. This means that the molecular weight has grown on exposure to a stretching hydrodynamic field.

Data on determining the polymer's molecular weight by the absolute methods, which point, at least, to the absence of reduction in the molecular weight in the process of degradation of polymer solutions, can be explained, assuming the initiation of intramolecular branchings (crosslinkings) by the longitudinal hydrodynamic field. They can be of both chemical $[63,67]$ and physical [68] nature. The formation of supramolecular structures in the PEO and PAM solutions due to the change in the phase diagram under the action of stretching flows can be evidence in favor of the physical nature of the interactions.

The generation of a small amount of insoluble supramolecular particles in diluted polymer solutions [67], presumably, should fully be attributed to the formation of intermolecular cross-linkings of a chemical nature. The absence of the reduction in the PEO and PAM molecular weight, according to the data of light scattering and of the sedimentationdiffusion analysis, points to the fact that polymer cross-linkings are mainly formed between portions of one and the same strongly deformed coiled macromolecule. The formation of intramolecular cross-linkings leads to a reduction in the dimensions of coiled macromolecules and in their kinetic flexibility, i.e., to a loss of deformability in the longitudinal velocity field.

The degradation of polymer solutions is independent of the time of their residence in the flow with a transverse velocity gradient and is fully determined by the value of the longitudinal velocity gradient and by the time of its action on the solution. In the hydrodynamic field with a longitudinal velocity gradient, the highest stability of an HPAM solution to exposure to the hydrodynamic fi eld is manifested in a neutral medium despite the maximum dimensions of polymer coils.

Data on the deformation action of a convergent hydrodynamic fi eld on macromolecules with variation in their initial dimensions due to the temperature, the ionic force, and the medium's $\mathrm{pH}$ demonstrate that a necessary condition for the degradation of polymer solutions in 
the hydrodynamic field is its strong deformation action on the coiled molecules.

Experimental investigation of the degradation of PEO, PAM, and HPAM aqueous solutions under stretching-flow conditions has enabled us to state that the obtained results are unambiguously interpreted under the assumption of initiation of intra- and intermolecular branchings (cross-linkings) by the hydrodynamic field, which dominate over the rupture of macromolecules.

The established flow diagram of the process of degradation of polymer solutions enables us to formulate the physicochemical principles of the behavior of macromolecules under the conditions of composite hydrodynamic fields and to outline ways of raising the efficiency of their use in the oil and gas technologies.

\section{Specific of Polymer Solution Flow in the Sloted-Porous Reservoir}

Today, there are a lot of methods to enhance oil recovery from reservoirs, but most of them are environmentally hazardous. The most effective methods that allow extracting up to $40-45 \%$ of oil are the methods involving injection of acid, alkalis and other active chemicals into the formation [69]. But in the process of oil production contamination of the environment is taking place. Great damage is caused to it by chemical reagents during their injection into the reservoir or delivery to the field [70,71].

Because of this, the solution to the problem of improving the environmental efficiency of oil recovery methods is now relevant and timely. Among the methods of increasing oil displacement from reservoirs, the method of polymer flooding meets the most environmental requirements, but is not always highly efficient [9].

One of the main issues that needsto be solved during the development of the technology for increasing oil recovery from formations by using polymer solutions is to determine the optimal flow regime in the fractured-porous reservoir. Technological indicators when using polymer solutions to increase oil recovery from the reservoir are calculated being based on the condition of satisfying the inequality 


$$
\dot{\varepsilon} \cdot \theta_{c} \leq D e_{c r}
$$

where $\theta_{\mathrm{c}}-$ relaxation time of the polymer solution; $\dot{\varepsilon}-$ longitudinal velocity gradient.

Expression (1) is a criterion for the transition of a macromolecular coil from a hydrodynamically impermeable "segmental gel", where a significant part of the segments is screened, to a flowing "segmental solution" in which all segments already hydrodynamically interact with the solvent. When performing the above inequality in polymer solutions (polyethylene oxide, polyacrylamide, etc.) in the flow with a longitudinal velocity gradient, dynamic supramolecular structures are formed [34,72, 73]. Expression (6.1) should be interpreted as the Deborah number because the inverse value of the longitudinal gradient of velocity is nothing but the time scale of the stream [74].

Thus, the calculation covers calculating the relaxation time (characteristic time) of the polymer solution and the longitudinal velocity gradient taking place which is realized during of polymer solutions flow in the fractured-porous reservoir. According to the experimentally obtained data, the critical Deborah number for water solutions of polyethylene oxide (PEO) is 2.5 [2].

We obtained the following equation to define the characteristic relaxation time of the polymer solution (viscoelastic fluid)

$$
\theta_{c}=\left\{\begin{array}{ll}
\theta_{0} e^{k} & \text { at } k<1 \\
\theta_{0} \frac{e^{k^{2 / 3}}}{k^{1 / 3}} & \text { at } k>1
\end{array},\right.
$$

where $[\eta] 0$ - characteristic viscosity, $C$ - concentration of polymer in solution, and $[\eta] 0 \cdot C=k$. Dependence $\theta_{\mathrm{c}} / \theta_{0}$ on $[\eta] 0 \cdot C$ for PEO of two different molecular masses in the water as shown in fig. 6.1.

The solid line shows the course of the dependence obtained for equation (6.2). We can see that the points obtained during the experiments for the corresponding concentration regions lie satisfactorily on the estimated curve. 


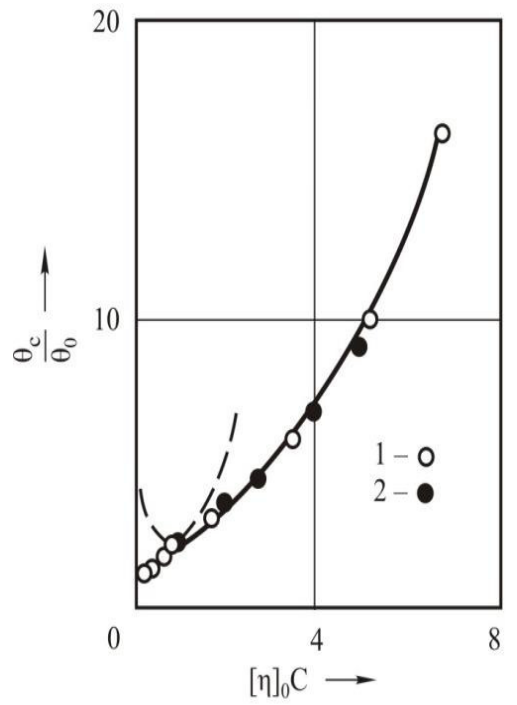

Fig. 6.1. Profiles of $\theta_{\mathrm{c}} / \theta_{\mathrm{c}}$ for varying concentration $[\eta]_{0} C$ for different PEO in solution MPEO: $1-4 \cdot 10^{6} ; 2-2,5 \cdot 10^{6}$

So, substituting the known molecular characteristics of the polymer into equation (6.2) we can calculate the relaxation time of polymer solutions. The effect of temperature in this equation is taken into account by the temperature dependence $\theta_{\mathrm{c}}$ i $k$.

At the same time, research literature to our knowledge doesn't highlight any analytical equations for calculating a longitudinal velocity gradient in the input area of the slot. To calculate the longitudinal velocity gradient of the polymer solution flow in the input area of the slot, it is necessary, taking into account its viscoelastic properties, to analyze the flow of polymer solution through the slotedporous reservoir.

Problem Statement. Polymer solutions possess viscoelastic properties [75-77]. Therefore, when polymer solution flows through the slot, to evaluate deformation characteristics of the flow (stream functions, distributions of the longitudinal velocity gradient and normal stress) resulting in manifestation of abnormal (compared with the behaviour of the ordinary fluid) effects, we can use a well-known Maxwell's fluid model 75-78] with the Jaumann derivative [79]. 
We chose this model because, according to Lodge [78], the study of non-linear, non-permanent, from the view of Lagrange, currents of viscoelastic fluids doesn't add any new information to the already obtained by studying homogeneous or quasi-homogeneous shear deformations. In his opinion, “... the only reason for detailed calculations of different types of non-linear currents is to make sure that they are practically implemented." This Lodge's statement can be interpreted in such a way, that there is no need for new rheological equations to describe convergent flows (in the input area of the slot); it's enough to use the equations of a steady Couette flow [75], or, at least, to determine whether they can explain the specific of polymer solution convergent flow in the sloted-porous reservoir.

Solution. To describe steady streamflow in the incompressible environments, we use the following classical equations [74,79]

- the continuity equation

$$
\mathcal{V}_{, i}^{i}=0,
$$

- the Cauchy motion equation

$$
\rho \mathcal{V}^{k} \mathcal{V}_{, k}^{i}=-g^{i k} P_{\cdot k}+T_{, k}^{i j},
$$

where $g^{\text {ik }}-$ metric tensor, and $T_{k}^{i j}$ is determined by covariant differentiation $T^{i j}$

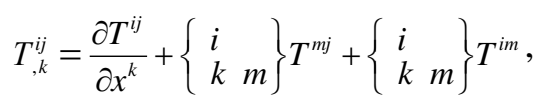

where $\left\{{ }_{k}^{i} m\right\}$ are the Christoffel symbols expressed by the dependence

$$
\left\{{ }_{k}^{i} m\right\}=\frac{1}{2} g^{i \ell}\left(\frac{\partial g_{k \ell}}{\partial x^{m}}+\frac{\partial g_{m \ell}}{\partial x^{k}}-\frac{\partial g_{k n}}{\partial x^{\ell}}\right) .
$$

Having designated $\theta_{\mathrm{c}}$ to relaxation time and $\eta_{\mathrm{c}}-$ to viscosity, we can write the structural rheological equation of the Maxwell's fluid model [75-80]

$$
T^{i j}+\theta_{c} \frac{D_{j} T^{i j}}{D t}=2 \eta_{c} D^{i j},
$$

where $D_{j} / D_{t}$ - the Jaumann derivative described by the equation

$$
\frac{D_{j} T^{i j}}{D t}=\frac{\partial T^{i j}}{\partial t}+V^{k} T_{k}^{i j}-W_{k}^{i} T^{k j}-T^{i k} W_{k}^{j},
$$

in which 


$$
\begin{aligned}
& D_{k m}=\frac{1}{2}\left(\mathcal{V}_{k, m}+\mathcal{V}_{m, k}\right), \\
& W_{k m}=\frac{1}{2}\left(\mathcal{V}_{k, m}-\mathcal{V}_{m, k}\right) .
\end{aligned}
$$

Analyze the case, where the incompressible fluid moves between two parallel planes and flows through the slot which length is considerably longer than width. The flow is flat and stationary. Fig. 6.2 shows the shape of the slot of the the sloted-porous reservoir and Cartesian coordinates.

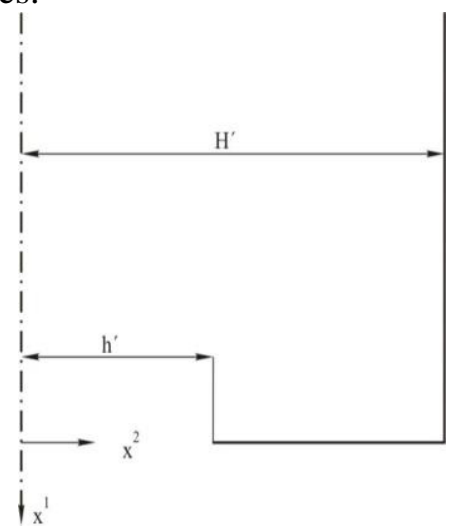

Fig. 6.2. The shape of the slot and Cartesian coordinates

The components of the metric tensor in Cartesian coordinates are

$$
\begin{aligned}
& g_{11}=g_{22}=1, \\
& g_{12}=g_{21}=0 .
\end{aligned}
$$

The Christoffel symbols $\left\{\begin{array}{l}i \\ k\end{array} \quad \begin{array}{l}m\end{array}\right\}$ equal zero since the components of the metric tensor $g_{\text {ik }}$ are not coordinate-dependent. Let us express this in a dimensionless form, introducing the following quantities into equations (6.3), (6.4) and (6.5)

$$
\begin{array}{rlrl}
x_{1}^{*} & =\frac{x^{1}}{H^{\prime}}, & x_{2}^{*} & =\frac{x^{2}}{H^{\prime}}, \\
V_{1}^{*} & =\frac{V^{l}}{\bar{u}}, & V_{2}^{*}=\frac{\mathcal{V}^{2}}{\bar{u}},
\end{array}
$$




$$
\begin{array}{rlrl}
T_{11}^{*} & =\frac{H^{\prime}}{\eta_{c} \bar{u}} T^{l l}, & T_{22}^{*}=\frac{H^{\prime}}{\eta_{c} \bar{u}} T^{22}, \\
T_{12}^{*}=\frac{H^{\prime}}{\eta_{c} \bar{u}} T^{12}, & T_{21}^{*}=\frac{H^{\prime}}{\eta_{c} \bar{u}} T^{21}, \\
P^{*}=\frac{H^{\prime}}{\eta_{c} \bar{u}} P, &
\end{array}
$$

where $\overline{\mathrm{u}}$-average flow velocity; $2 \mathrm{H}^{\prime}$ - slot width. Considering transformations (3), (4), (5), they are reduced to

$$
\begin{aligned}
& \frac{\partial v_{1}^{*}}{\partial \mathrm{x}_{1}^{*}}+\frac{\partial \mathcal{V}_{2}^{*}}{\partial \mathrm{x}_{2}^{*}}=0 \\
& \operatorname{Re}\left(\mathcal{V}_{1}^{*} \frac{\partial \mathcal{V}_{1}^{*}}{\partial \mathrm{x}_{1}^{*}}+\mathcal{V}_{2}^{*} \frac{\partial \mathcal{V}_{1}^{*}}{\partial \mathrm{x}_{2}^{*}}\right)=-\frac{\partial \mathbf{P}^{*}}{\partial \mathrm{x}_{1}^{*}}+\frac{\partial \mathrm{T}_{11}^{*}}{\partial \mathrm{x}_{1}^{*}}+\frac{\partial \mathrm{T}_{12}^{*}}{\partial \mathrm{x}_{2}^{*}} \\
& \operatorname{Re}\left(\mathcal{V}_{1}^{*} \frac{\partial \mathcal{V}_{2}^{*}}{\partial \mathbf{x}_{1}^{*}}+\mathcal{V}_{2}^{*} \frac{\partial \mathcal{V}_{2}^{*}}{\partial \mathbf{x}_{2}^{*}}\right)=-\frac{\partial \mathbf{P}^{*}}{\partial \mathbf{x}_{2}^{*}}+\frac{\partial \mathbf{T}_{21}^{*}}{\partial \mathbf{x}_{1}^{*}}+\frac{\partial \mathbf{T}_{22}^{*}}{\partial \mathbf{x}_{2}^{*}},(6.8 b) \\
& T_{11}^{*}+W e\left\{\begin{array}{l}
\mathcal{V}_{1}^{*} \frac{\partial T_{11}^{*}}{\partial x_{1}^{*}}+\mathcal{V}_{2}^{*} \frac{\partial T_{11}^{*}}{\partial x_{2}^{*}}- \\
-\frac{1}{2}\left(\frac{\partial \mathcal{V}_{1}^{*}}{\partial x_{2}^{*}}-\frac{\partial \mathcal{V}_{2}^{*}}{\partial x_{1}^{*}}\right)\left(T_{12}^{*}+T_{21}^{*}\right)
\end{array}\right\}=2 \frac{\partial \mathcal{V}_{1}^{*}}{\partial x_{1}^{*}}, \\
& T_{22}^{*}+W e\left\{\begin{array}{l}
\mathcal{V}_{1}^{*} \frac{\partial T_{22}^{*}}{\partial x_{1}^{*}}+v_{2}^{*} \frac{\partial T_{22}^{*}}{\partial x_{2}^{*}}+ \\
+\frac{1}{2}\left(\frac{\partial \mathcal{V}_{1}^{*}}{\partial x_{2}^{*}}-\frac{\partial \mathcal{V}_{2}^{*}}{\partial x_{1}^{*}}\right)\left(T_{12}^{*}+T_{21}^{*}\right)
\end{array}\right\}=2 \frac{\partial \mathcal{V}_{2}^{*}}{\partial x_{2}^{*}},
\end{aligned}
$$




$$
\begin{gathered}
\left.T_{12}^{*}+W e\left\{\begin{array}{l}
\mathcal{V}_{1}^{*} \frac{\partial T_{12}^{*}}{\partial x_{1}^{*}}+\mathcal{V}_{2}^{*} \frac{\partial T_{12}^{*}}{\partial x_{2}^{*}}+ \\
\frac{1}{2}\left(\frac{\partial \mathcal{V}_{1}^{*}}{\partial x_{2}^{*}}-\frac{\partial \mathcal{V}_{2}^{*}}{\partial x_{1}^{*}}\right)\left(T_{11}^{*}-T_{22}^{*}\right)
\end{array}\right)\right\}=\frac{\partial \mathcal{V}_{1}^{*}}{\partial x_{2}^{*}}+\frac{\partial \mathcal{V}_{2}^{*}}{\partial x_{1}^{*}}, \\
T_{21}^{*}+W e\left\{\begin{array}{l}
\mathcal{V}_{1}^{*} \frac{\partial T_{21}^{*}}{\partial x_{1}^{*}}+\mathcal{V}_{2}^{*} \frac{\partial T_{21}^{*}}{\partial x_{2}^{*}}+ \\
+\frac{1}{2}\left(\frac{\partial V_{1}^{*}}{\partial x_{2}^{*}}-\frac{\partial \mathcal{V}_{2}^{*}}{\partial x_{1}^{*}}\right)\left(T_{11}^{*}-T_{22}^{*}\right)
\end{array}\right)=\frac{\partial \mathcal{V}_{1}^{*}}{\partial x_{2}^{*}}+\frac{\partial \mathcal{V}_{2}^{*}}{\partial x_{1}^{*}},
\end{gathered}
$$

where $\operatorname{Re}=\frac{\rho \bar{u} H^{\prime}}{\eta_{c}}$ - the Reynolds number; $W e=\frac{\theta_{c} \bar{u}}{H^{\prime}}$ - the Weissenberg number.

If we restrict ourselves to a flow in which the inertial terms can be neglected, then the left side of equation (6.8) will equal zero. Applying the continuity equation (6.7), we introduce the stream function

$$
v_{1}^{*}=\frac{\partial \psi}{\partial \mathrm{x}_{2}^{*}}, v_{2}^{*}=-\frac{\partial \psi}{\partial \mathrm{x}_{1}^{*}}
$$

If we assume that the fluid flow at the slot input area of the oil sloted-porous reservoir has the Poiseuille velocity profile, the velocity on the surface of the solid wall (adhesion condition) equals zero, and the flow velocity is constant, the boundary conditions will take the following form

$$
\begin{gathered}
x_{1}^{*}=-\infty, \quad \mathcal{V}_{1}^{*}=\frac{3}{2}\left(1-\mathrm{x}_{2}^{* 2}\right), \quad V_{2}^{*}=0, \\
x_{1}^{*}=0, \quad 0 \leq \mathrm{x}_{2}^{*} \leq \mathrm{h}^{*}, \quad V_{1}^{*}=V_{0}^{*} \quad V_{2}^{*}=0, \\
x_{1}^{*}=0, \quad \mathrm{~h}^{*} \leq \mathrm{x}_{2}^{*} \leq 1, \quad \mathcal{V}_{1}^{*}=V_{2}^{*}=0, \\
x_{2}^{*}=0, \quad \frac{\partial \mathcal{V}_{1}^{*}}{\partial \mathrm{x}_{2}^{*}}=V_{2}^{*}=0,
\end{gathered}
$$




$$
x_{2}^{*}=1, \quad V_{1}^{*}=V_{2}^{*}=0,
$$

where $v_{0}^{*}=$ const, determined by the expendable velocity; $h^{*}$ dimensionless value, equals $\mathrm{h}^{\prime} / \mathrm{H}^{\prime} ; 2 \mathrm{H}^{\prime}$ - slot width.

It is necessary to solve equations (6.7), (6.8), and (6.9), according to the boundary conditions (6.11), to determine the flow and stress fields, according to the boundary conditions (6.11). It is not possible to solve these equations in a general form; therefore, we restrict ourselves to slow flows. In this case, we can not only neglect the inertial terms but also assume that the Weisenberg number is less than one.

Let's recall that the Weissenberg number characterizes the rate of the viscoelastic properties of the fluid in a shear flow. In the case under consideration, we have a complex flow with both shear and longitudinal velocity gradients. As the velocity of the outflow through the slot increases, as proven in [23], the proportion of the longitudinal (stretching) flow increases, and the shear decreases. Therefore, it is more appropriate, instead of the Weissenberg number, to use a Deborah number, which characterizes the rate of viscoelastic properties in a stretching flow [74]. Nevertheless, for stationary flows, the ratio is $D e / W e=\operatorname{Re}^{0,75}[74,81]$, which means that both criteria, $W e$ and $D e$, are interconnected within geometrically similar flows.

Therefore, for restrictions, imposed on this flow, in the same way as in [79], we can write the velocities, stress, and stream functions as the decomposition by number $W e$

$$
\begin{aligned}
& \mathcal{V}_{i}^{*}=\mathcal{V}_{i}^{(0)}+W e \mathcal{V}_{i}^{(1)}+W e^{2} \mathcal{V}_{i}^{(2)}+\ldots, \\
& P^{*}=P^{(0)}+W e P^{(1)}+W e^{2} P^{(2)}+\ldots, \\
& T_{i j}^{*}=T_{i j}^{(0)}+W e T_{i j}^{(1)}+W e^{2} T_{i j}^{(2)}+\ldots, \\
& \psi^{*}=\psi^{(0)}+W e \psi^{(1)}+W e^{2} \psi^{(2)}+\ldots .
\end{aligned}
$$

Substituting (6.12) into equations (6.7), (6.8), (6.9), and the boundary conditions (6.11), we will put the equation in order with respect to the Weissenberg number. Now we can write down the terms of equations that do not include the Weissenberg number: 


$$
\begin{gathered}
\frac{\partial \mathcal{V}_{1}^{(0)}}{\partial \mathrm{x}_{1}^{*}}+\frac{\partial \mathcal{V}_{2}^{(0)}}{\partial \mathrm{x}_{2}^{*}}=0, \\
\frac{\partial \mathrm{T}_{11}^{(0)}}{\partial \mathrm{x}_{1}^{*}}+\frac{\partial \mathrm{T}_{12}^{(0)}}{\partial \mathrm{x}_{2}^{*}}=\frac{\partial \mathrm{P}^{(0)}}{\partial \mathrm{x}_{1}^{*}}, \\
\frac{\partial \mathrm{T}_{21}^{(0)}}{\partial \mathrm{x}_{1}^{*}}+\frac{\partial \mathrm{T}_{22}^{(0)}}{\partial \mathrm{x}_{2}^{*}}=\frac{\partial \mathrm{P}^{(0)}}{\partial \mathrm{x}_{2}^{*}}, \\
\mathrm{~T}_{11}^{(0)}=2 \frac{\partial \mathcal{V}_{1}^{(0)}}{\partial \mathrm{x}_{1}^{*}}, \quad \mathrm{~T}_{22}^{(0)}=2 \frac{\partial \mathcal{V}_{2}^{(0)}}{\partial \mathrm{x}_{2}^{*}}, \\
\mathrm{~T}_{12}^{(0)}=\frac{\partial \mathcal{V}_{1}^{(0)}}{\partial \mathrm{x}_{1}^{*}}+\frac{\partial \mathcal{V}_{2}^{(0)}}{\partial \mathrm{x}_{1}^{*}}, \\
\mathrm{~T}_{21}^{(0)}=\frac{\partial \mathcal{V}_{1}^{(0)}}{\partial \mathrm{x}_{1}^{*}}+\frac{\partial \mathcal{V}_{2}^{(0)}}{\partial \mathrm{x}_{1}^{*}}, \\
\frac{\partial \psi^{(0)}}{\partial \mathrm{x}_{2}^{*}}, \quad \mathcal{V}_{2}^{(0)}=-\frac{\partial \psi^{(0)}}{\partial \mathrm{x}_{1}^{*}}
\end{gathered}
$$

Boundary conditions

$$
\begin{array}{ll}
x_{1}^{*}=-\infty, & V_{1}^{(0)}=\frac{3}{2}\left(1-x_{2}^{* 2}\right), \quad V_{2}^{(0)}=0, \\
x_{1}^{*}=0, \quad 0 \leq x_{2}^{*} \leq h^{*}, \quad & V_{1}^{(0)}=V_{0}^{*}, \quad v_{2}^{(0)}=0, \\
x_{1}^{*}=0, \quad h^{*} \leq x_{2}^{*} \leq 1, \quad v_{1}^{(0)}=V_{2}^{(0)}=0, \\
x_{2}^{*}=0, \quad \frac{\partial V_{1}^{(0)}}{\partial x_{2}^{*}}=v_{2}^{(0)}=0, \\
x_{2}^{*}=1, \quad v_{1}^{(0)}=v_{2}^{(0)}=0 .
\end{array}
$$

Considering equation (6.13), by expressing $\frac{\partial \mathrm{P}^{(0)}}{\partial \mathrm{x}_{1}^{*}}, \frac{\partial \mathrm{P}^{(0)}}{\partial \mathrm{x}_{2}^{*}}$ through $\psi^{(1)}=0$ and its derivatives and excluding $\mathrm{P}^{(0)}$, we obtain

$$
\left(\frac{\partial^{2}}{\partial x_{1}^{* 2}}+\frac{\partial^{2}}{\partial x_{2}^{* 2}}\right) \psi^{(0)}=0
$$

For boundary conditions (14) the solution of the equation (6.15) 
describes the flow of the ordinary fluid.

$$
\psi^{(0)}=\psi^{(0)}\left(x_{1}^{*}, x_{2}^{*}\right)
$$

By substituting equation (6.12) into equation (6.9) and grouping the terms with the Weissenberg number in the first power, we obtain

$$
\begin{aligned}
& T_{11}^{(1)}=\frac{1}{2}\left(\frac{\partial \mathcal{V}_{1}^{(0)}}{\partial x_{2}^{*}}-\frac{\partial \mathcal{V}_{2}^{(0)}}{\partial x_{1}^{*}}\right)\left(T_{12}^{(0)}+T_{21}^{(0)}\right)+ \\
& +2 \frac{\partial \mathcal{V}_{1}^{(1)}}{\partial x_{1}^{*}}-\mathcal{V}_{1}^{(0)} \frac{\partial T_{1}^{(0)}}{\partial x_{1}^{*}}-\mathcal{V}_{2}^{(0)} \frac{\partial T_{11}^{(0)}}{\partial x_{2}^{*}}, \\
& T_{22}^{(1)}=2 \frac{\partial \mathcal{V}_{2}^{(1)}}{\partial x_{2}^{*}}-\frac{1}{2}\left(\frac{\partial \mathcal{V}_{1}^{(0)}}{\partial x_{2}^{*}}-\frac{\partial \mathcal{V}_{2}^{(0)}}{\partial x_{1}^{*}}\right)\left(T_{12}^{(0)}+T_{21}^{(0)}\right) V_{1}^{(0)} \frac{\partial T_{22}^{(0)}}{\partial x_{1}^{*}}-\mathcal{V}_{2}^{(0)} \frac{\partial T_{22}^{(0)}}{\partial x_{2}^{*}} \text {, } \\
& T_{12}^{(1)}=\frac{\partial V_{1}^{(1)}}{\partial x_{2}^{*}}+\frac{\partial \gamma_{2}^{(1)}}{\partial x_{1}^{*}}-\frac{1}{2}\left(\frac{\partial \mathcal{V}_{1}^{(0)}}{\partial x_{2}^{*}}-\frac{\partial \mathcal{V}_{2}^{(0)}}{\partial x_{1}^{*}}\right)\left(T_{11}^{(0)}-T_{22}^{(0)}\right)-\gamma_{1}^{(0)} \frac{\partial T_{12}^{(0)}}{\partial x_{1}^{*}}-\mathcal{V}_{2}^{(0)} \frac{\partial T_{12}^{(0)}}{\partial x_{2}^{*}},(6 \\
& T_{21}^{(1)}=\frac{\partial \gamma_{1}^{(1)}}{\partial x_{2}^{*}}+\frac{\partial \gamma_{2}^{(1)}}{\partial x_{1}^{*}}-\frac{1}{2}\left(\frac{\partial \gamma_{1}^{(0)}}{\partial x_{2}^{*}}-\frac{\partial \gamma_{2}^{(0)}}{\partial x_{1}^{*}}\right)\left(T_{11}^{(0)}-T_{22}^{(0)}\right)-\gamma_{1}^{(0)} \frac{\partial T_{21}^{(0)}}{\partial x_{1}^{*}}-\gamma_{2}^{(0)} \frac{\partial T_{21}^{(0)}}{\partial x_{2}^{*}},
\end{aligned}
$$

where $\mathcal{V}_{i}^{(0)}, T_{i j}^{(0)}$ - the velocity and stress components of the terms of equations having the Weissenberg number in a zero power, respectively; they are known.

In the similar way, converting the continuity equation, the equation of motion and the boundary conditions, we obtain

$$
\begin{aligned}
& \frac{\partial \mathcal{V}_{1}^{(1)}}{\partial \mathrm{x}_{1}^{*}}+\frac{\partial \mathcal{V}_{2}^{(1)}}{\partial \mathrm{x}_{2}^{*}}=0, \\
& \frac{\partial \mathrm{T}_{11}^{(1)}}{\partial \mathrm{x}_{1}^{*}}+\frac{\partial \mathrm{T}_{12}^{(1)}}{\partial \mathrm{x}_{2}^{*}}=\frac{\partial \mathrm{P}^{(1)}}{\partial \mathrm{x}_{1}^{*}}, \\
& \frac{\partial \mathrm{T}_{21}^{(1)}}{\partial \mathrm{x}_{1}^{*}}+\frac{\partial \mathrm{T}_{22}^{(1)}}{\partial \mathrm{x}_{2}^{*}}=\frac{\partial \mathrm{P}^{(1)}}{\partial \mathrm{x}_{2}^{*}} .
\end{aligned}
$$

Boundary conditions 


$$
\begin{array}{ll}
x_{1}^{*}=-\infty, & \mathcal{V}_{1}^{(1)}=\mathcal{V}_{2}^{(1)}=0, \\
x_{1}^{*}=0, & \mathcal{V}_{1}^{(1)}=V_{2}^{(1)}=0, \\
x_{2}^{*}=1, & \mathcal{V}_{1}^{(1)}=V_{2}^{(1)}=0, \\
x_{2}^{*}=0, & \mathcal{V}_{1}^{(1)}=V_{2}^{(1)}=0 .
\end{array}
$$

The stream function (6.10) takes the following form

$$
v_{1}^{(1)}=\frac{\partial \psi^{(1)}}{\partial x_{2}{ }^{*}}, \quad v_{2}^{(1)}=-\frac{\partial \psi^{(1)}}{\partial x_{1}^{*}}
$$

Considering(6.19) and excluding equation (6.17), we obtain this equation

$$
\left(\frac{\partial^{2}}{\partial x_{1}^{* 2}}+\frac{\partial^{2}}{\partial x_{2}^{* 2}}\right)^{2} \psi^{(1)}=0 .
$$

The solution of equation (6.20) with boundary conditions (6.18) has the form $\psi^{(1)}=0$, consequently, the terms of the equation with the Weissenberg number in the first power do not affect the velocity distribution. However, as we can see from equation (6.16), stress $T_{11}^{(1)}, T_{22}^{(1)}, T_{12}^{(1)}, T_{21}^{(1)}$ prove the influence of the elasticity of the terms of the equation with the Weissenberg number in the first power. By substituting equation (6.12) into equation (6.19), taking into account that $\mathcal{V}_{1}^{(1)}=\mathcal{V}_{2}^{(1)}=0$, and grouping the terms containing the Weissenberg number in the second power, we obtain

$$
\begin{aligned}
& T_{11}^{(2)}=2 \frac{\partial \mathcal{V}_{1}^{(2)}}{\partial x_{1}^{*}}+\frac{1}{2}\left(\frac{\partial \mathcal{V}_{1}^{(0)}}{\partial x_{2}^{*}}-\frac{\partial \mathcal{V}_{2}^{(0)}}{\partial x_{1}^{*}}\right)\left(T_{12}^{(1)}+T_{21}^{(1)}\right)-\mathcal{V}_{1}^{(0)} \frac{\partial T_{11}^{(1)}}{\partial x_{1}^{*}}-\mathcal{V}_{2}^{(0)} \frac{\partial T_{11}^{(1)}}{\partial x_{2}^{*}}, \\
& T_{22}^{(2)}=2 \frac{\partial V_{2}^{(2)}}{\partial x_{2}^{*}}-\frac{1}{2}\left(\frac{\partial V_{1}^{(0)}}{\partial x_{2}^{*}}-\frac{\partial V_{2}^{(0)}}{\partial x_{1}^{*}}\right)\left(T_{12}^{(1)}+T_{21}^{(1)}\right)-\mathcal{V}_{1}^{(0)} \frac{\partial T_{22}^{(1)}}{\partial x_{1}^{*}}-V_{2}^{(0)} \frac{\partial T_{22}^{(1)}}{\partial x_{2}^{*}}, \\
& T_{12}^{(2)}=\frac{\partial V_{1}^{(2)}}{\partial x_{2}^{*}}+\frac{\partial V_{2}^{(2)}}{\partial x_{1}^{*}}-\frac{1}{2}\left(\frac{\partial V_{1}^{(0)}}{\partial x_{2}^{*}}-\frac{\partial V_{2}^{(0)}}{\partial x_{1}^{*}}\right)\left(T_{11}^{(1)}-T_{22}^{(1)}\right)-V_{1}^{(0)} \frac{\partial T_{12}^{(1)}}{\partial x_{1}^{*}}-V_{2}^{(0)} \frac{\partial T_{12}^{(1)}}{\partial x_{2}^{*}} \text {, } \\
& T_{21}^{(2)}=\frac{\partial V_{1}^{(2)}}{\partial x_{2}^{*}}+\frac{\partial V_{2}^{(2)}}{\partial x_{1}^{*}}-\frac{1}{2}\left(\frac{\partial V_{1}^{(0)}}{\partial x_{2}^{*}}-\frac{\partial V_{2}^{(0)}}{\partial x_{1}^{*}}\right)\left(T_{11}^{(1)}-T_{22}^{(1)}\right)-V_{1}^{(0)} \frac{\partial T_{21}^{(1)}}{\partial x_{1}^{*}}-V_{2}^{(0)} \frac{\partial T_{21}^{(1)}}{\partial x_{2}^{*}} \text {. }
\end{aligned}
$$


Once in equations (6.17), (6.18) and (6.19) index (1) was substituted by (2), the continuity equations, the equations of motion, the boundary conditions and the stream function took the same form.

Therefore, excluding $\mathrm{P}^{(2)}$, we'll get

$$
\begin{aligned}
& \left(\frac{\partial^{2}}{\partial x_{1}^{* 2}}+\frac{\partial^{2}}{\partial x_{2}^{* 2}}\right)^{2} \psi^{(2)}=\frac{\partial^{2} V_{1}^{(0)}}{\partial x_{2}^{*} \partial x_{1}^{*}}\left(\frac{\partial A}{\partial x_{1}^{*}}+\frac{\partial A}{\partial x_{2}^{*}}\right)+ \\
& +\frac{\partial \mathcal{V}_{1}^{(0)}}{\partial x_{2}^{*}}\left(\frac{\partial^{2} A}{\partial x_{1}^{* 2}}+2 \frac{\partial^{2} T_{12}^{(l)}}{\partial x_{2}^{*} \partial x_{1}^{*}}\right)+\frac{\partial \mathcal{V}_{2}^{(0)}}{\partial x_{1}^{*}}\left(\frac{\partial^{2} A}{\partial x_{2}^{* 2}}-2 \frac{\partial^{2} T_{12}^{(l)}}{\partial x_{1}^{*} \partial x_{2}^{*}}\right)- \\
& -C \frac{\partial^{2} B}{\partial x_{1}^{*} \partial x_{2}^{*}}-\frac{B}{2}\left(2 \frac{\partial^{2} C}{\partial x_{1}^{*} \partial x_{2}^{*}}-\frac{\partial^{2} A}{\partial x_{2}^{* 2}}+\frac{\partial^{2} A}{\partial x_{1}^{* 2}}\right)- \\
& -\frac{\partial B}{\partial x_{1}^{*}}\left(\frac{\partial C}{\partial x_{2}^{*}}+\frac{\partial A}{\partial x_{1}^{*}}\right)+\frac{\partial B}{\partial x_{2}^{*}}\left(\frac{\partial A}{\partial x_{2}^{*}}-\frac{\partial C}{\partial x_{1}^{*}}\right)+\frac{\partial^{2} V_{1}^{(0)}}{\partial x_{2}^{* 2}} \cdot \frac{\partial T_{12}^{(l)}}{\partial x_{1}^{*}}+ \\
& +\frac{\partial^{2} V_{2}^{(0)}}{\partial x_{2}^{* 2}} \cdot \frac{\partial T_{12}^{(1)}}{\partial x_{2}^{*}}-\frac{\partial^{2} \mathcal{V}_{1}^{(0)}}{\partial x_{1}^{* 2}} \cdot \frac{\partial T_{12}^{(1)}}{\partial x_{1}^{*}}-\frac{\partial^{2} \mathcal{V}_{2}^{(0)}}{\partial x_{1}^{* 2}} \cdot \frac{\partial T_{21}^{(1)}}{\partial x_{2}^{*}}+ \\
& +2 \frac{\partial \mathcal{V}_{2}^{(0)}}{\partial x_{2}^{* 2}}\left(\frac{\partial^{2} T_{12}^{(1)}}{\partial x_{2}^{* 2}}+\frac{\partial^{2} T_{12}^{(1)}}{\partial x_{1}^{* 2}}\right)+\frac{A}{2}\left(\frac{\partial^{2} B}{\partial x_{2}^{* 2}}-\frac{\partial^{2} B}{\partial x_{1}^{* 2}}\right)+ \\
& +\mathcal{V}_{1}^{(0)}\left(\frac{\partial^{3} T_{12}^{(1)}}{\partial x_{2}^{* 2} \partial x_{1}^{*}}-\frac{\partial^{3} T_{12}^{(1)}}{\partial x_{1}^{* 3}}+\frac{\partial^{3} A}{\partial x_{1}^{* 2} \partial x_{2}^{*}}\right)+ \\
& +\mathcal{V}_{2}^{(0)}\left(\frac{\partial^{3} T_{12}^{(1)}}{\partial x_{2}^{* 3}}-\frac{\partial^{3} T_{12}^{(1)}}{\partial x_{2}^{* 2} \partial x_{2}^{*}}+\frac{\partial^{3} A}{\partial x_{2}^{* 2} \partial x_{1}^{*}}\right) \cdot
\end{aligned}
$$

where $A=T_{11}^{(1)}-T_{22}^{(1)} ; \quad B=\frac{\partial \mathcal{V}_{1}^{(0)}}{\partial x_{2}^{*}}-\frac{\partial \mathcal{V}_{2}^{(0)}}{\partial x_{1}^{*}} ; \quad C=T_{12}^{(1)}+T_{21}^{(1)}$.

Since the right-hand side of equation (6.22) is known, so by solving equation (6.22) that comprises the boundary conditions, we will obtain the terms of the equation with the Weissenberg number in the second power. These terms characterize the distribution of velocities and stresses. It should be noted that, in contrast to [79], the righthand side of equation (6.22) contains derivatives of the order that is higher than in [79].

Results of the numerical simulation of the Maxwell fluid flow through the slot. Figs. 6.3 and 6.4 visualise the stream function when 
ordinary $(W e=0)$ and Maxwell $(W e=0.1)$ fluids flow through the slot. Obviously, as the channel compression ratio decreases, the stream function experiences an increasing impact at the inlet slot.

When the Maxwell fluid flows through a simulated slotted chamber, there arises the circulation zone (fig. $3 b$ ) which extends from the right corner of the channel to the slot and occupies a triangular area. The flow lines form the inlet flow. Consequently, a decrease in the channel compression ratio (as well as an increase in $W e$ ) results in the input flooded jet.
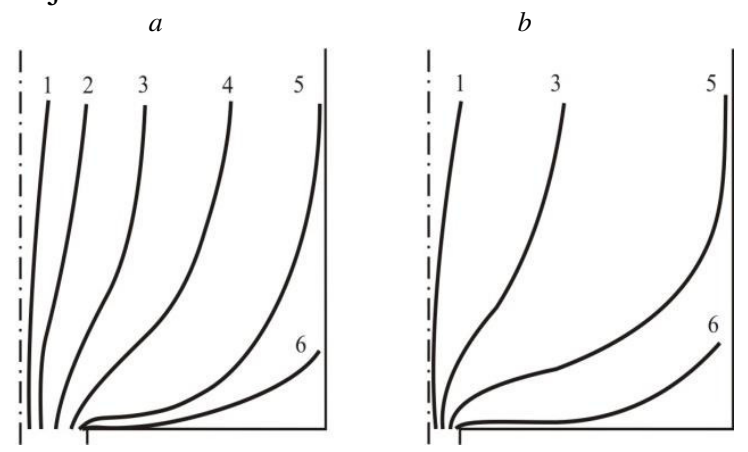

Fig. 6.3. Thestream function, when flowingt hroughthe slot of the Newtonianfluid $\mathrm{We}=0, \Psi: 1-0,125,2-0,375,3-0,625$, $4-0,875,5-0,9715,6-1,0$ $a-\mathrm{h}^{\prime} / \mathrm{H}^{\prime}-0,2 ; b-\mathrm{h}^{\prime} / \mathrm{H}^{\prime}-0,07$

$c$
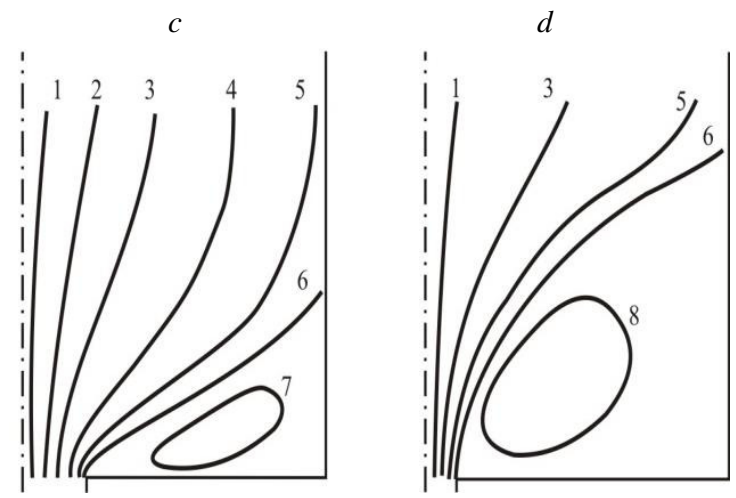

Fig. 6.4. The stream function, whenflowingthroughtheslot of the Newtonianfluid $\mathrm{We}=0,1, \Psi: 1-0,125,2-0,375,3-0,625$, $4-0,875,5-0,9715,6-1,0,7-1,01,8-1,1 ; a-\mathrm{h}^{\prime} / \mathrm{H}^{\prime}-0,2 ; b-\mathrm{h}^{\prime} / \mathrm{H}^{\prime}-0,07$ 
Fig. 6.5 shows the distribution of the dimensionless longitudinal velocity gradient along the flow axis when ordinary (curves 1 and 2) and Maxwell (curve 3) fluids flow into the slot. We can see that the maximum value of the velocity gradient in the flow of an ordinary fluid is reached at the distance $3 h^{\prime}$ i $\left(x_{1}^{*}=1,5\right)$ from the slot for the compression ratios of 0.2 and 0.07 , respectively. The flowing fluid acquires the viscoelastic properties what shifts the maximum on the curve $\dot{\varepsilon}^{*}=f\left(x_{1}^{*}\right)$ to the region of large $X^{*}$ and decreases the value of $\dot{\varepsilon}_{\text {max }}^{*}$.

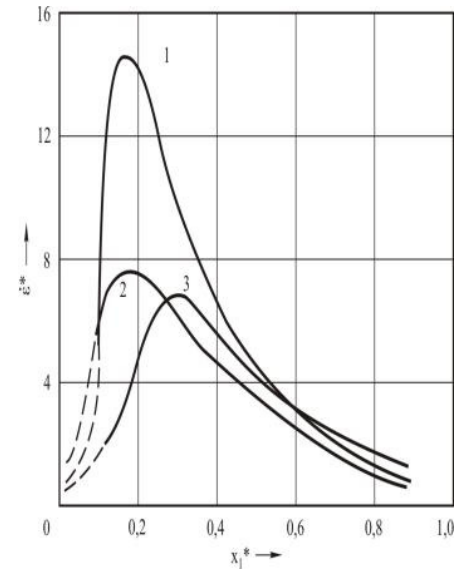

Fig. 6.5. The distribution of the dimensionless longitudinal velocity gradient along the axis of the fluid flowing into the slot

$1: \mathrm{h}^{\prime} / \mathrm{H}^{\prime}=0,07, \mathrm{We}=0$;

$2: \mathrm{h}^{\prime} / \mathrm{H}^{\prime}=0,2, \mathrm{We}=0$

$3: \mathrm{h}^{\prime} / \mathrm{H}^{\prime}=0,2, \mathrm{We}=0,1$

A comparison of the experimental data obtained in $[2,23,33,79]$ with the results of the numerical simulations shows that the simulated flow lines and the distribution of the velocity gradient correspond to the experimentally obtained results for relatively low velocities.

The distribution of dimensionless

normal stress for the channel compression ratio of 0.07 and the Weissenberg number 0.1 is shown in fig. 6.6.

The results properly visualise the experimental data on the distribution of isochromes in the input area of the slot (fig. 6.6b and [82]).

As in the polystyrene-bromoform system under consideration both the polymer and the solvent had the same refractive indices, the obtained lines of equal birefringence value (isochromes) inside the input flooded jet are proportional to the first difference of normal stresses. 

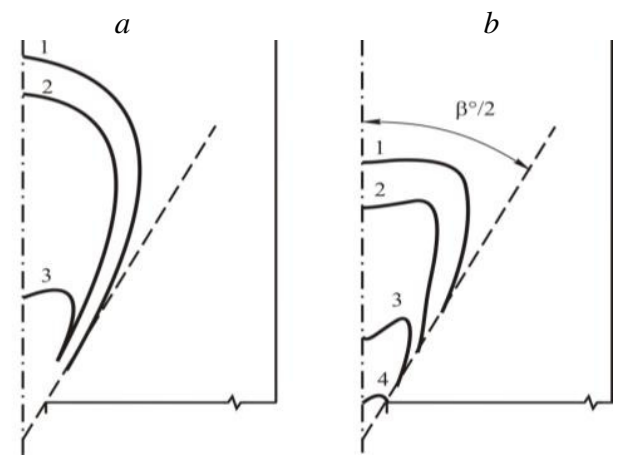

Fig. 6.6. The distribution of dimensionless normal stresses $(a)$ and isochromes (b) in the input area of a slot The angular half-width of the stream $\left(\beta^{\circ} / 2\right)-30^{\circ}$;

$$
\begin{gathered}
a-\mathrm{We}=0,1, h^{\prime} / H^{\prime}=0,07 ; \mathrm{T}^{*}: 1-5,2-7,3-45 ; \\
\quad b-\Delta \mathrm{n}: 1-10^{-4} 2-7 \cdot 10^{-4} 3-15 \cdot 10^{-4} 4-62 \cdot 10^{-4}
\end{gathered}
$$

The obtained results indicate that the numerical method of the convergent flow analysis of Maxwell's highly viscoelastic fluid can be used to calculate the longitudinal velocity gradients realized in the inlet area of cracks during the flow of polymer solutions in the porous-fractured reservoirs. It should be noted, however, that in the general case it is necessary to take into account the influence of the entryangle into the crack, which can be done by considering the problem in rectilinear inclined or curvilinear coordinates. At the same time, the analysis of the viscoelastic fluid flow in the porousfracturedreservoir is sharply complicated.

The conducted analysis of the viscoelastic fluid leakage through the fissure has defining value, first of all, for confirming the interpretation of experimental data proposed in $[11,33,83]$, which characterize special aspects of the convergent flow of aqueous polymer solutions in the inlet area of the hole or fracture.

The calculation results verify the ideas obtained from the experimental solution of this problem about the strain-stress state of polymer macromolecules (liquid elements) during polymer flow in the inlet area of the fissure in the oil reservoir and confirm the possibility of using numerical analysis of convergent polymer flow for calculating longitudinal velocity gradients in the inlet area of the fissure, and can also serve as additional substantiation of the proposed in $[10,86]$ mechanism for increasing oil recovery from reservoirs by using polymer solutions. 


\section{Conclusion}

Starting to summarize this work it is necessary to notice that polymers need to be used namely as polymers, i.e. not there where they replace something, but where they are irreplaceable. Such statement does not exclude application of polymers as materials in the usual meaning of this word. In popular articles authors still are biased to treat polymers as "materials of the future". The word "materials" dominates as though the only application of polymers is to be materials for engineering and machine designs, fibres, organic glasses, etc.

Such approach has immediate consequences leading to two extremes. The first of them reduces physics of polymers to physical materials technology at best, and chemistry - to the helper of chemical technology. The second extreme, which in some research circles turned to be a reaction on the first one, is characterized by "flight" into molecular biology, since polymeric molecules (macromolecules of DNA, RNA, etc.) are a basis of all alive.

All this leads to the situation when important technical (technological) problems to be solved due to polymers specific place among other substances remain unsettled.

We should note [84] that the fundamental property of polymers as a special form of substance condensation is their, i.e. ability in corresponding conditions to a limited (technology) or unlimited (evolution) entropy fall in some local system. Act of polymerization is something more fundamental than usual phase transition as starting from this point the system in principle may acquire ability to spontaneous self-complication and self-improvement.

Anti-entropability particularly has ties with individualization, or macromolecules distinctness, their ability to be carriers or transmitters (or transformers) of information and at last their ability for dynamic realization of information recorded on them, in particular, is connected. "Tomorrow" of polymer systems is connected with implementation of these properties in technics, medicine, genetics and other areas.

Polymers also differ from other substances by the fact that macromolecules are very big and are capable to change their form. This variety of molecular and super-molecular morphological forms in polymers and their solutions can be used widely in technics. 
Description of all possible applications of polymer systems would withdraw us too far aside from the main objective of this work. We'll enumerate only those applications that are most important for technics today. First of all they are new technological principles of system preparation for polymerization and transition from "usual" materials to anisotropic systems.

Polyfoams have big internal surface and consequently can be used for linking considerable quantities of substances possessing certain effect - effectors. For example, one of such effectors is haemoglobin that connects and transports oxygen. Haemoglobin is immobilized in foam-polyurethane that catches oxygen like fish gills. Using such haemo-sponge a diver can stay under water much longer than with a usual aqualung. There's also a possibility for a more attractive variant: decomposition of water takes place in polyfoam resulting in oxygen release. In both variants it is not an aqualung any more, i.e. "a water lung" (lat. aqua - water and Engl. lung - a lung), but "artificial gills".

Immobilization of haemoglobin in foam-polyurethane is only one of numerous examples showing possibilities of linkage, transportation and delivery of effectors by means of polymers. Effectors can be tied physically or chemically with a polymer surface, be grasped by polymeric particles, encapsulated by polymers or chemically sewed with them. The type of immobilization defines quantity and speed of effector release.

Microencapsulation is probably the most important process. It was discovered in 1953 by a scientific worker of "National Cash Register Company" who managed to cover small droplets of oil with a thin layer of water-containing-gelatin with its further hardening. Thus received gelatin microcapsules had diameter from 1 micron and above.

Other polymers can be used instead of gelatin. Not only oil droplets, but also many other liquid and solid materials can be encapsulated, for example, dyes, vitamins, bleaching agents, aromatic additives, herbicides, insecticides, medicines, fertilizers, adhesive materials, etc. Microcapsules are also used for manufacturing "carbon-free paper". Appearance of such paper made unnecessary the usage of carbon paper. Microcapsules are pasted to the underside of the original copy. When writing on the face side of any form capsules break with ink release to the duplicate. 
The encapsulated material can be released from a polymer cover not only under mechanical effect, but in other ways too. Capsules can resolve with the help either physical or chemical processes.

In the past well soluble fertilizers were applied after definite time intervals. However these fertilizers are easily washed off with rains into lakes and rivers causing eutrophication. Any plot of land should be treated with fertilizers during the whole period of vegetation Microencapsulation is likely to become very useful under these circumstances. If a polymer capsule is slightly permeable for water, fertilizer is used up during the whole period of vegetation. Similar processes take place in so-called bioorganic fertilizers. Inorganic effectors are encapsulated by means of organic materials that yield in slow decay with inorganic compounds release.

Encapsulation plays especially important role in pharmacology. First of all encapsulating materials ensure dosed medicine supply into the human body protecting it from overdosing. Similarly, application of microencapsulation to prolong dissolution period for special sewing agents in polymer solution was offered by us as one of the stages to implement technology of creating waterproof screens in the oil and gas technologies $[2,86]$.

The problem to decrease of hydrodynamic drag reduction of oil flow in pipelines by polymer additions and improvement of important technological methods when cleaning liquids from colloiddisperse impurities may also be settled with the use of polymers.

Therefore we proceed from the thesis that polymers need to be used namely as polymers when settling technical problems. This allowed us design a technological decisions for use of solutions of polymers in the oil and gas technologies.

References

1. Pogrebnyak V. G., Voloshin V. S. (2017) Enerhozberezhennia i efekt Tomsa: za redaktsiieiu Yu. F. Ivanyuta. Monografiya. Kiev, Ukraine: Osvita Ukrainy. $440 \mathrm{~s}$.

2. Pogrebnyak V. G., Voloshin V. S. (2010) Ecological Technology of Creating Waterproof Screens. Monografia. Donetsk, Ukraine: Knowledge. 482 p.

3. El'yashevich G. K., Frenkel'S. Ya. (1980) Thermodynamics of orientation of polymer solutions and melts, in: A. Ya. Malkin and S. P. Papkov (Eds.), Orientation Phenomena in Polymer Solutions and Melts. Moscow: Khimiya.Pp. 9-90. 
4. Pogrebnyak V. G., Perkun I. V., Naumchuk M.V. (2011) Sposib osvitlennia harchovykh ridyn polimernymy flokuliantamy. Pat. 57600 Ukraine, MPK C12H 1/02 (2006.01). № U2010 08323, Biul. 5.

5. Pogrebnyak V.G., Perkun I.V., Pogrebnyak A.V. (2013) Parametry flokuliatsiinoho protsesu, shcho zabezpechuiut pidvyshchennia spozhyvnykh vlastyvostei ta yakist yabluchnoho soku pid chas osvitlennia. Tovaroznavchyi vistnyk.Zb. nauk. prats. Lutsk: LTU. Vyp. 6. Ss. 205-212.

6. Jones W. H. and Ho S. P. (1979) The flow of dilute aqueous solutions of macromolecules in various geometries. VII. Mechanisms of resistance in porous media. J. Phys.Vol. 12, No. 3. Pp. 383-393.

7.Boyko V. S., Kondrat R. M., Yaremchuk R. C. (1996) Handbook of oil and gas science. L'viv: Svit. 620 p.

8. Korsakova N. K., Penkovsky V. I., Altuninaand L. K., Kuvshinov V. A. (2016) Redistribution of filtration flows by thermo gel at boundary water flooding of oil reservoirs. AIP Conf. Proc. Tomsk. Pp. 1783-1784.

9. Malkin A. Y., Arinstein A., Kulichikhin V. G. (2014) Polymer extension flows and instabilities. Progress in Polymer Science. Vol. 39, No. 5. Pp. 959-978.

10. Pogrebnyak V. G., Pisarenko A. A. (1999) Deformation effects in case of a flow with stretching of polymer solutions. Turbulence and Shear flow phenomena, Santa Barbara, California, Banerjee S., Eaton J. K. editors. New York. Pp. 13451350.

11. Pogrebnyak V. G., Ivanyuta Yu. F. and Frenkel S. Y. (1992) The structure of the hydrodynamic field and distortions of the molecular shape of flexible polymers under free-converging flow conditions. Polymer Sci. USSR. Vol. 34,No. 3. Pp. 270-273.

12. Ferry J. D. (1980) Viscoelastic properties ofpolymers. John Wiley \& Sons.641 p.

13. Malkin A. Y. (2009) Status of Polymer Rheology: achievements and problems series. Polymer Sci. ser. A. Vol. 51, No. 1. Pp. 106-136.

14. Baranov V. G., BrestkinYu. V., Agranova S. A., Pinkevich V. N. (1986) The behavior of macromolecules polystyrene in "viscosities" of a good solvent. Polymer Sci. ser. B. Vol. 28, No. 11. Pp. 841-843.

15.Bazelevich A. V., Entov V. M., Karpov A. V. et al. (1991) The Relaxation Time of Polymer Solutions: Methods of Measurement and Some ofIts Applications. Moscow: Instit. of Appl. Mech. of the Russian Academy of Sciences. 42 p.

16. Chornyy M. I., Chornyy O. M., Metoshop I. M., Kuzin I. M. (2013) Heolohichni osnovy rozkrittia i viprobuvannia produktyvnykh plastiv.Navchalnyjiposibnyk. Ivano-Frankivsk: Wydawnictwa IFNTUNG. $306 \mathrm{~s}$.

17. Ivanyuta Yu. F., Naumchuk N. V., Pogrebnyak V. G.,Frenkel' S. Ya. (1985) Flow structure of aqueous solutions of polyethylene oxide in the inlet region of short capillaries. Journal of Engineering Physics. Vol. 49,No.4. Pp. 1192-1197.

18. Diment O. N., Kazanskii K. S., Miroshnikov A. M. (1976) Glikoli i drugiye proizvodnye okisey etilena i propilena. Monografiya. Moscow: Khimiya. $374 \mathrm{~s}$.

19. Salyanov V. I., Skuridin S. G., Lortkipanidze G. B. et al. (1978) Relation between molekular-strukture of aqueous-solutions of polyethylene-glycol and 
comhaction of double-standed DNK-molecules.Molekulyarnaya Biologiya. Vol.12, No. 3. Pp. 485-495.

20.Brestkin Yu. V., Ahranova S. F., D'iakova N. E. et al. (1989) Birefringence effects ofpolymer-solutions in hydrodynamic fields. Vysokomolekulyarnye Soedineniya. Ser. B. Vol. 31, No.11. Pp. 844-846.

21. Brestkin Yu. V. (1987) Dynamic coil-extended chain phase transition in the longitudinal field. Acta Polymerica. Vol. 38, No.8. Pp. 470-477.

22. Keller A., Odell J. A. (1985) The extensibility of macromolecules in solutions, a new focus for macromolecular science. Colloid. Polym. Sci. Vol. 263, No. 3. Pp. 181-201.

23. PogrebnyakA. V., PerkunI. V., PogrebnyakV. G. (2017) Degradation of Polymer Solutions in a Hydrodynamic Field with a Longitudinal Velocity Gradient. Journal of Engineering Physics and Thermophysics. Vol. 90, No. 5.Pp. 1219-1224. DOI: $10.1007 / \mathrm{s} 10891-017-1677-8$.

24. Pogrebnyak A. V., Perkun I. V., Pogrebnyak V.G., Shimanskii V. Y. (2021) Thermal Effects in the Flow of a Polymer Aqueous Solution Through a Hydrocutting Jet-Forming Head. Journal of Engineering Physics and Thermophysics. Vol. 94, No.1. Pp. 137-142. DOI:10.1007/s10891-021-02281-1.

25. Povkh I. L., Pogrebnyak V. G., Toryanik A. I. (1979) Relation between molecular structure of polyethyleneoxideand drag reduction.Journal of Engineering Physics. Vol. 37, No.4. Pp. 1131-1136.

26. Kuzmin R. A., Tihomirov R. A.,Kravchenko D. V.(2002) O preimushchestvakh gidrorezaniya materialov struey zhidkosti s dobavkami vodorastvorimykh polimerov. Aerokosmicheskie tekhnologiii obrazovanie narubezhe vekov: Materialy Vserossiyskoy nauchno-tekhnicheskoy konferenczii. Rybinsk:Wydawnictwa RGATA. Chast` 2. Ss. 53-54.

27. Pogrebnyak V. G., Naumchik N. V. (1995) On the hydrodynamic activity of polymers in high-velocity flows. Inzhenerno-FizicheskiiZhurnal. Vol. 68, No. 1.Pp. 146-148.

28. Atanov H. A. (1987) Gidroimpulsnye ustanovki dliya razrusheniiy. Monografiya. Kiev: Wydawnictwa Vyshcha shkola. 152 s.

29. Brenner V. A., Jabin A. B., Pushkarev A. E., Sycheholev M. M. (2000) Gidrostrujny`e tekhnologii v promyshlennosti. Gidromekhanicheskoe razrushenie gornykh porod. Monografiya. Moskva: Wydawnictwa Akad. Gornykh nauk. 343 s.

30. Azzari P., Rjanova A. B., Svitasheva K. K. (1981) Ellipsometriya i polyarizovannyj svet.Monografiya. Moskva: WydawnictwaMir. $583 \mathrm{~s}$.

31. Kudin A. M. (1972) On the sizes of associates in solutions of polymers that reduce the hydrodynamic resistance. Hydromekhanika. Kiev: Wydawnictwa Science Dumka. Is. 20. Ss.82-87.

32. Pogrebnyak V. G., Chudyk I. I., Perkun I. V. (2021) High-efficiency Casing Perforation Oil and Gas Wells. SOCAR Proceedings. Reservoir and Petroleum Engineering. No. 2. Pp. $112-120$.

http://dx.doi.org/10.5510/OGP2021SI200578.

33. Pogrebnyak A., Chudyk I., Pogrebnyak V., PerkunI. (2019) Coiluncoiled chain transition of polyethylene oxide solutions under convergent flow. Chemistry and Chemical Technology. Vol. 13, No.4. Pp. 465-470. 
DOI:10.23939/chcht13.04.465.

34. Pogrebnyak V. G., Naumchik N. V. (1992)Dynamic structurization in solutions of hydrodynamically active polymers. Journal of Engineering Physics and Thermophysics.Vol. 63, No. 2. Pp. 763-765.DOI: 10.1007/BF00861695

35. Kryvenko G., Perkun I.,Pogrebnyak V. G. (2017) The nature of Hydrodynamic Drag Reduction of Oil Flow in Pipelines by Polymer Additions. Petroleum Science and Engineering. Vol. 2, No.3. Pp. 79-85.

DOI: $10.11648 /$ j.pse.20170102.12.

36. PogrebnyakV. G., IvanyutaYu. F., Naumchuk I. V. (1991) Unrolling of macromolecules under wall turbulence conditions. Inzhenerno-FizicheskiiZhurnal. Vol.61, No.6. Pp. 925-927.

37. Cantwell B. I. (1981) Organized motion in turbulent flow. Ann. Rev. Fluid. Mech. No.13. p. 457-519.

38. Bredshou P. (1974) Vvedenie v turbulentnostieeizmerenie. Monografiya. Moskva: Wydawnictwa Mir. 278 s.

39. Pogrebnyak V. G., PogrebnyakA. V., PerkunI. V. (2021) Maxwell fluid flow in system supplying hydrodynamically active polymer to boundary layer of streamlined object.Mathematical Modeling and Computing. Vol. 8, No. 1. Pp. 5868. DOI:10.23939/mmc2021.01.058.

40. Frenkel'S. Ya., El'iashevich H. K., Panov Yu. N. (1970) Kontsentrirovannyerastvorypolimerov Uspekhi khimii i fiziki polimerov. Moskva: Khimiya. Pp. 87-138.

41. Pilipenko V. N. (1980) Vliyanie dobavok na pristennye turbulentnye techeniya. Itogi nauki I tekhniki. Ser. Mekhanikazhidkostiigaza. Moskva: Wydawnictwa VINITI. No.15. Pp. 156-257.

42. Hlesdorf P., Prigojin I. (2003) Termodinamicheskaya teoriya struktury, ustoychivosti i fluktuatsii. Monografiya. Moskva: WydawnictwaURSS. 280 p.

43. Abdalla A. Y., Japper-Jaafar A., Mohd Fakrul Radzi Mohd Radzi (2016) Effects of Polymer Addition to a Newtonian Solvent in Horizontal Pipe Flow. Journal of Applied Sciences. No 16. Pp. 271-278.

44. Motier I. F., Prilutski P. I. (1985) Gase histories of polymer draq reduction in crude oil lines. Pipe lines industry. Vol. 62, No 6. Pp. 33, 34, 37.

45. Pogrebnyak V. G., Ivanyuta Yu. F. (1998) Experimental research of the influence of conditions of polymer admission to the boundary layer on a drop of turbulent friction. Proc. of the Intern. Symp. on Seawater Drag Reduction. Newport, Rhade Island USA, S. 1. Pp. 295-297.

46. Polimer expands crude line capacity. (1985) Pipe line industry. Vol. 63, No.1. Pp. 71-72.

47. Al-Yaaria M., Soleimanib A., Abu-Sharkha B., Al- Mubaiyedha U., Alsarkhic A. (2009) Effect of drag reducing polymers on oil-water flow in a horizontal pipe. International Journal of Multiphase Flow. No 35. Pp. 516-524.

48. Durst F., Ray S., Unsal B.and Bayoumi O. A. (2005) The development lengths of laminar pipe and channel flows. J. Fluids Eng. No 127. Pp. 1154-1160.

49. Beaty W. K., Ionston R. L., Rramer R. L., Warnack L. G., Wheeler G. R (1984) Offshore crude oil production increased by drag reducers. Drag Reduct. 3 rd int. Conf. Bristol. F1 -F14. 
50. Nesyn G. V., Manzhai V. N., Suleimanov Yu. V., Stankevych V. S., Konovalov K. V.(2012) Mechanism of action, evaluation of efficiency and features of obtaining polymeric anti-turbulent additives for transport of hydrocarbon liquids. Polymer Science, Ser. A. Vol. 54, No 1. Pp. 65-72.

51. Nikulin V. A. (2014) About one model of heat transfer in wall- adjacent turbulence. Ser. 1. Mechanics and Physics: Fundamental and applied research. Vol.1, No. 43. Pp. 4-12.

52.Stupin O. B., Symonenko O. P., Aslanov P. V. (2013)Hydrodynamic activity of water- soluble polymer compositions and perspectives of their application in energy saving and ecology. Visnyk of National University "Lviv Polytechnic": Thermal power. Engineering environment. Automation. No. 758. Pp. 85-96.

53. Symonenko A. P. (2012) Improving the efficiency of firefighting equipment by applying the hydrodynamic activity of water - soluble polymer compositions. Compilation of scientific works of the National Civil Protection University of Ukraine: Problems of Fire Safety. Is.32. Pp. 195-206.

54.Liberanore M. W., Pollauf E. J., McHungh A. J. J. (2003) Shear - induced structure formation is solutions of drag reducing polymers. Non-Newton. Fluid Mech. Vol. 113, No. 2-3. Pp. 193-208.

55. Pogrebnyak V. G., Pisarenko A. A.(2002) Solutions of Polymers under the Conditions of Wall Turbulence. Mechanism of Drag Reduction. Intern. J. of Fluid Mech. Research.Vol. 29, No. 6. Pp. 130-138.

56. Wu J., Fruman D. H., Tulin M. P. (1978) Drag reduction by polymer diffusion at high Reynolds numbers. J. ofHydronautics.Vol. 12, July. Pp. 134-136.

57. Fruman D. H.and Galivel P. (1979) Anomalous effects connected with ejection of polymer, reducing resistance, in turbulent boundary layers of pure water. Technical papers from the Symposium on Viscous Drag Reduction, Ed. By Gary R. Hough, Vought Advanced Technology Center, Dallas, Texas. Vol.72. Pp. 12331241 .

58. Vdovin A. V. and Smolyakov A. V. (1978) Diffusion of solutions in a turbulent boundary layer. Zh. Prikl. meh. iteh. Fiz. No. 2. Pp. 66-73.

59. Casale A.and Porter R. S. (1978-1979) Polymer Stress Reactions. Vols. 12, Academic Press, New York. 269 s.

60.Henmann T. J. Polymer degradation and stabilization. Polym. News. Vol.9, No.10. Pp. 309-311.

61. Soules D., Fernando R. H. and Glass J. (1986) Mechanical stability of water-soluble carbohydrate polymers. Polym. Mater. Sci. Eng. Div. No. 53. Pp. 555559.

62. Brestkin Yu. V. et al. Destruction of polystyrene macromolecules in a strong longitudinal hydrodynamic field. Vysomol. Soedin. Ser. B.Vol. 31, No.7. Pp. 506-510.

63. Vyshkina T. V. (1988) Chemical Transformations of Polyacrylamide in the Hydrodynamic Field. Candidate's Dissertation in Chemical Sciences. Kiev: NU named after T. Shevchenko. $110 \mathrm{~s}$.

64. Povkh I. L.(1976) Technical Hydromechanics. Leningrad: Mashinostroenie. $502 \mathrm{~s}$. 
65.Smol'skii B. M., Shul'man Z. P. and Gorislavets V. M. (1970) Rheodynamics and Heat Transfer of Nonlinearly Viscoelastic Materials. Minsk: Nauka i Tekhnika. $466 \mathrm{~s}$.

65. Tsvetkov V. N., Éskin V. E. and Frenkel' S. Ya. (1964) Structure of Macromolecules in Solutions. Moscow: Nauka.719 s.

67. Klenin V. I. (1995) Thermodynamics of Systems with Flexible Polymers. Monografiya. Saratov: Saratovskii Univ. 736 s.

68. El'yashevich G. K.andFrenkel' S. Ya. (1980) Thermodynamics of orientation of polymer solutions and melts, in: A. Ya. Malkin and S. P. Papkov (Eds.), Orientation Phenomena in Polymer Solutions and Melts.Moscow: Khimiya, Pp. 9-90.

69. Boiko V. S., Kondrat R. M., Yaremiichuk Z. S. (1996)Dovidnyk z naftohazovoispravy. Lviv: IFNTUNH. $620 \mathrm{~s}$.

70.Kachurin A., Sattarov R., AyupovaD., GabdullinaA.(2011) Improvement of the technology of strata oil recoveryenhancementusingSoftPusher Polyacrylamide in the fields of LUKOIL -Western Siberia OOO. Oil Industry. No. 8. Pp. 126-128.

71. Bondarenko A. V., Kudryashova D. A. (2015) The application of hydrodynamic modeling for predictive effectiveness assessment of polymer floodingtechnologyonMoskudinskoyefield. Oil Industry. No.10.Pp. 102-105.

72. Pogrebnyak V. G. (2005) Polymer Macromolecules as a Tool for Studying Wall-Adjacent Turbulence Flow. Proceedings of the 2nd International Symposium on Seawater Drag Reduction. Busan, Korea: ASERC. Pp. 79-90.

73. De Gennes P. G.(1974) Coil-stretch thransition of dilute flexible polymers under ultrahigh velocity gradients. J. Chem. Phys.Vol. 60, No 12. Pp. 5030-5042.

74.Astarita J., Marucci J. (1978) Osnovyi gidromehaniki nenyutonovskih zhidkostey. Moscow: Mir. 309 s.

75. Vinogradov G. V., Malkin A. Ya. (1977) Reologiya polimerov. Moscow: Khimiya. $438 \mathrm{~s}$.

76. Ferri J. (1963) Vyazkouprugiesvoystva polimerov.Moscow: Inostr. lit. 535 s.

77. Kristensen R. (1974) Vvedenie v teoriyu vyazkouprugosti. Moscow: Mir. $338 \mathrm{~s}$.

78. Lodge A. S. (1969) Elastichnyie zhidkosti. Vvedenie v reologiyu konechnodeformiruemyih polimerov. Moscow: Nauka. $463 \mathrm{~s}$.

79. Nakamura K. (1978) Medlennoe istechenie vyazkouprugoy zhidkosti po konicheskomu kanalu. Senk'i kikay gakkaysi. Vol. 31, No.8. P. 49-55.

80. Voytkunskiy Ya. I., Amfilohiev V. V., Pavlovskiy V. A. (1970) Uravnenie dvizheniya zhidkosti s uchetom ee relaksacionnykh svojstv. Sb. Nauch. tr. Leningr. korablestr. ins-t. No. 69, Ss.19-25.

81. Hintse I. O. (1963) Turbulentnost. Mehanizm i teoriya. Moscow: Phiz.-mat. Izdat. $680 \mathrm{~s}$.

82. Brestkin Yu. V., Amribakhshov A. A., Kholmuminov A. A., Frenkel S.Y. (1988) Razvorachivanie makromolekul pri shodyaschemsya techenii. Izv. AN UzSSR. Seriya phiz.-mat. nauk. No. 6. Pp. 80-84.

83. Pogrebnyak A. (2017) The Elastic Deformations and Anomalously High Cutting Ability of a Polymer Solution Jet. Scientific journal of the Ternopil Ivan Puluj National Technical University. Vol. 1, No.85. Pp. 89-94. 
84. Frenkel' S. Ya. (1973) Polimeryi. Problemyi, perspektivyi i prognozyi. Fizika segodnya i zavtra: za redaktsiieiu V. M.Tuchkevicha. Leningrad: Nauka. Ss.176-270.

85. Feynman R. P. (1966) The Development of the Space-Time View of Quantum Electrodynamics. Science. Vol. 153, No. 3737. Pp. 699-708.

86. Pogrebnyak V.G., Perkun I. V., Pogrebnyak A. V. (2016)Displacement of Oil from Porous Bed by the Oscillating Flow of Polymer Solution. American Journal of Science. Engineering and Technology. Vol. 1, No 2. Pp. 53-57.

https://doi.org/10.31713/m1107

\title{
TECHNOLOGY OF RAW MATERIAL ENRICHMENT OF INHOMOGENEOUS CARBONATE DEPOSITS AND EVALUATION OF ITS ENERGY EFFICIENCY
}

\section{Dreshpak O. S.}

Dnipro University of Technology, $\mathrm{PhD}$, associate professor, Department of Technological Engineering of Materials Processing, Ukraine

\section{Dreshpak N. S.}

Dnipro University of Technology, $\mathrm{PhD}$, associate professor, Department of Electrical Engineering, Ukraine

\section{Vypanasenko S. I.}

Dnipro University of Technology, DSc (Engineering), professor, Department of Power Engineering, Ukraine

\begin{abstract}
The article presents the results of solving the urgent scientific and practical problem of establishing regularities connecting the indicators of the efficiency of enrichment and classification of raw materials of heterogeneous limestone deposits with its initial characteristics, technological and design parameters of the classification equipment, substantiating rational screening modes on this basis. An assessment of the energy efficiency of the proposed technical solutions is given. The research was carried out at Dnipro University of Technology (Ukraine, Dnipro).

Technological lines for dry processing of limestone raw materials, which are used in quarries, ensure the production of marketable products of acceptable quality only for large grades of crushed stone in low humidity conditions. Small grades of crushed stone are not used and are sent to the dump. There is a need to improve the existing process of classification and enrichment of raw materials in order to improve the quality of the final product and expand, on this basis, the fractional com-
\end{abstract}

